\title{
Precision spectroscopy on atomic hydrogen
}

Präzisionsspektroskopie am atomaren Wasserstoff

Christian Godehard Parthey 



\title{
Precision spectroscopy on atomic hydrogen
}

Präzisionsspektroskopie am atomaren Wasserstoff

Christian Godehard Parthey

\author{
Dissertation \\ an der Fakultät für Physik \\ der Ludwig-Maximilians-Universität \\ München \\ vorgelegt von \\ Christian Godehard Parthey \\ aus Schlüchtern
}

München, den 02.11.2011 
Erstgutachter: Prof. Dr. Theodor W. Hänsch

Zweitgutachter: Prof. Dr. Martin Faessler

Tag der mündlichen Prüfung: 15.12.2011 
$f_{1 S-2 S}=2466061413187035(10) \mathrm{Hz}$

To my parents. 


\section{Contents}

Zusammenfassung $\quad$ xi

Abstract $\quad$ xiii

1 Introduction $\quad 1$

2 Theory 5

2.1 Dirac Energy . . . . . . . . . . . . . . . . . . . . 5

2.2 Lamb Shift . . . . . . . . . . . . . . . . . . . . 6

2.3 Hyperfine Splitting . . . . . . . . . . . . . . . . . . . 6

2.3.1 Hyperfine Splitting in an External Magnetic Field . . . . . . . . . . 7

3 Experimental Procedure $\quad 9$

3.1 Beam Apparatus . . . . . . . . . . . . . . . . . . . 9

3.1.1 Quench Laser Beam near $486 \mathrm{~nm}$ at the Entrance of the Excitation

Region . . . . . . . . . . . . . . . . 10

3.1.2 High-Efficiency Lyman- $\gamma$ Detector . . . . . . . . . . . . . . . . . 10

3.1.3 Enhancement-Cavity Transmission . . . . . . . . . . . . . . . 11

3.2 Laser System . . . . . . . . . . . . . . . . . . . . . . 11

3.2 .1 Dye Laser . . . . . . . . . . . . . . . . . . . . . . . . . . . . . . . . . . . . . 11

3.2.2 Extended-Cavity Diode Laser ECDL1 . . . . . . . . . . . . . . . . . 12

3.2.3 Extended-Cavity Diode Laser ECDL2 . . . . . . . . . . . . . . 13

3.3 Frequency Measurement . . . . . . . . . . . . . . . . . . 14

3.3.1 Frequency Comb System . . . . . . . . . . . . . . . 15

3.3.2 Maser and Radio Frequency Multiplication . . . . . . . . . . . . . 15

3.3.3 Maser Calibration . . . . . . . . . . . . . . . . . . 16

4 The $1 S-2 S$ Hydrogen / Deuterium Isotope Shift 19

4.1 Introduction . . . . . . . . . . . . . . . . . . . . 19

4.2 Experimental Procedure . . . . . . . . . . . . . . . . 20

4.2.1 Differential Frequency Measurement with the Maser as Flywheel . . 20

4.2.2 First Measurement Run with Dye Laser and ECDL1 . . . . . . . . 20

4.2.3 Second Measurement Run with ECDL1 exclusively . . . . . . . . . 21 
4.2.4 Data Taking Protocol . . . . . . . . . . . . . . . . . 21

4.3 Systematic Uncertainties . . . . . . . . . . . . . . . . . . . . . . . . . . . . . . . . . . . . . .

4.3.1 Second Order Doppler effect . . . . . . . . . . . . . . 21

4.3 .2 dc Stark Shift . . . . . . . . . . . . . . . . . . 22

4.3 .3 ac Stark Shift . . . . . . . . . . . . . . . . . . . . . . . . . . . . . . . . . . . 23

4.3 .4 Pressure Shift . . . . . . . . . . . . . . . . . . 24

4.4 Result . . . . . . . . . . . . . . . . . . . . 25

4.5 Proton - Deuteron Charge Radius Difference . . . . . . . . . . . . . 26

5 Absolute Frequency Measurement 29

5.1 Introduction . . . . . . . . . . . . . . . . . . . . . . . . . . . . 29

5.2 Experimental Summary . . . . . . . . . . . . . . . . . . . 29

5.2.1 Beam Apparatus . . . . . . . . . . . . . . . . . . . 29

5.2.2 Laser System and Frequency Measurement . . . . . . . . . . . . . . 30

5.2 .3 Measurement Procedure . . . . . . . . . . . . . . . . . 30

5.3 Characterization of Uncertainties . . . . . . . . . . . . . . . 31

5.3.1 Quadratic ac Stark Shift due to the Spectroscopy Laser . . . . . . . 32

5.3 .2 Line Shape . . . . . . . . . . . . . . . . . 33

5.3.3 Second Order Doppler Effect . . . . . . . . . . . . . . . 34

5.3 .4 ac Stark Shift by $486 \mathrm{~nm}$ Quench Light . . . . . . . . . . . . . 36

5.3.5 Hyperfine Correction . . . . . . . . . . . . . . . . . 37

5.3 .6 dc Stark Shift . . . . . . . . . . . . . . . . . . . . . . . . . . . . . . . . 37

5.3 .7 Pressure Shift . . . . . . . . . . . . . . . . . . . . . . . . . . . . . . . . . .

5.3 .8 Zeeman Shift . . . . . . . . . . . . . . . . . . 37

5.3 .9 Blackbody Shift . . . . . . . . . . . . . . . 38

5.3.10 ac Stark Shift due to the Radio Frequency Discharge . . . . . . . . 39

5.3.11 Frequency Shift due to the Power-Switching Acousto-optic Modulator 40

5.3.12 Line Pulling by the $m_{F}=0$ Hyperfine Component . . . . . . . . . . 41

5.3.13 Higher Order Modes within the Enhancement Cavity . . . . . . . . 42

5.3 .14 Recoil Shift . . . . . . . . . . . . . . . . . 43

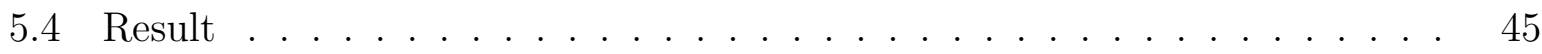

6 Second Absolute Frequency Measurement $\quad 47$

6.1 Introduction . . . . . . . . . . . . . . . . . . . . . . 47

6.2 Experimental Summary . . . . . . . . . . . . . . . . 47

6.2.1 Laser System and Frequency Calibration via the $900 \mathrm{~km}$ Fiber Link 47

6.2.2 Statistical Improvement with the High-Efficiency Lyman- $\alpha$ Detector 48

6.2.3 Measurement Process . . . . . . . . . . . . . . . . . . . . . 48

6.3 Summary of Uncertainties . . . . . . . . . . . . . . . . . . . . . 49

6.3.1 Additional Uncertainty of the Second Order Doppler Effect with the

New Lyman- $\alpha$ Detector . . . . . . . . . . . . . . . . . . 50

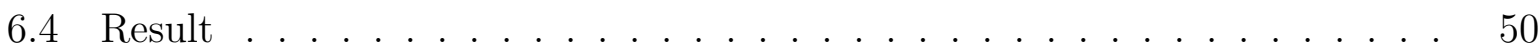

6.4.1 Agreement of both Absolute Frequency Measurements . . . . . . . 50 
6.5 Analysis of the Hydrogen Experiment within the SME Framework . . . . . 52

6.5.1 Introduction to the Standard Model Extensions (SME) . . . . . . . 52

6.5.2 The SME Coordinate System . . . . . . . . . . . . . . 53

6.5.3 Using the Hydrogen Experiment to Test Lorentz Boost Invariance . 53

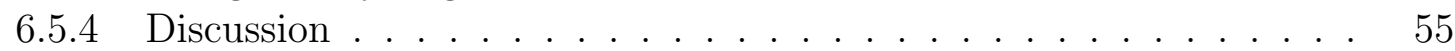

6.6 Constraint on the Drift of Fundamental Constants . . . . . . . . . . . . 55

7 Conclusion and Outlook $\quad 57$

7.1 Hydrogen / Deuterium Isotope Shift . . . . . . . . . . . . . . . . 57

$7.2 \quad 1 S-2 S$ Absolute Frequency . . . . . . . . . . . . . . . . . 58

A Testing the Frequency Comb and Radio Frequency Equipment $\quad 61$

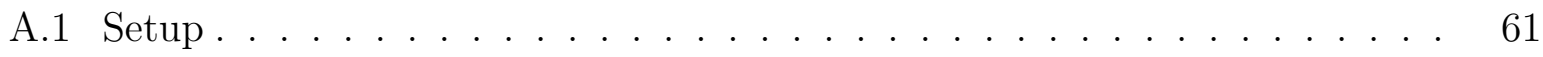

A.2 Cycle Slip Detection . . . . . . . . . . . . . . . 61

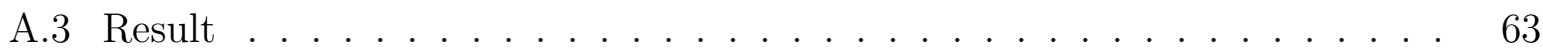

B Absolute Frequency of the Isotope Shift Hydrogen Data 65

C Analysis of Artificial Absolute Frequency Data $\quad 67$

C.1 Test of the Line Shape Model . . . . . . . . . . . . . . . . . . . . . . . . 67

C.2 Comparison of Simulation and Experiment . . . . . . . . . . . . . 69

C.2.1 Line width . . . . . . . . . . . . . . . . . . . 69

C.2.2 ac Stark shift . . . . . . . . . . . . . . . . 70

$\begin{array}{ll}\text { D Dye laser lock } & 73\end{array}$

E Collection of Experimental Parameters and Procedures $\quad 77$

$\begin{array}{lr}\text { Bibliography } & 81\end{array}$

$\begin{array}{lr}\text { Acknowledgments } & 91\end{array}$

$\begin{array}{ll}\text { List of Publications } & 95\end{array}$ 


\section{List of Figures}

2.1 Hydrogen level scheme . . . . . . . . . . . . . . . . . . . . . . . 7

2.2 Hydrogen level scheme in an external magnetic field . . . . . . . . . . . 8

3.1 Beam apparatus for two-photon spectroscopy on hydrogen / deuterium . . . 9

3.2 Schematic of the high-efficiency Lyman- $\alpha$ detector . . . . . . . . . . . . . . 11

3.3 Transmission of the enhancement cavity . . . . . . . . . . . . . . 12

3.4 Schematic of the ECDL laser system . . . . . . . . . . . . . . . 13

3.5 Schematic of the laser system with dye laser . . . . . . . . . . . . . 14

3.6 Allan deviations for the frequency components present in our experiments . 15

3.7 Fractional frequency difference of the MPQ maser versus FOM . . . . . . . 16

3.8 The frequency measurement using CSF1 and the optical fiber link . . . . 18

4.1 Characterization of stray electric fields _ . . . . . . . . . . . . . 22

4.2 The isotope shift: ac Stark shift interpolation . . . . . . . . . . . . . 24

4.3 The isotope shift plotted versus hydrogen and deuterium pressure . . . . . 25

4.4 Isotope shift result . . . . . . . . . . . . . . . . . . . 27

5.1 Absolute frequency measurement statistics . . . . . . . . . . . . . . 31

$5.2 \quad 1 S-2 S$ line profiles for various delays $\ldots \ldots \ldots \ldots \ldots \ldots \ldots$

5.3 Setup for measuring the $2 S$ velocity distribution . . . . . . . . . . . . 33

5.4 Measured $2 S$ velocity profiles and power dependence $\ldots \ldots \ldots \ldots$

5.5 Correction to the $1 S-2 S$ frequency for the May 2010 measurement . . . . . 35

5.6 Measurement of the Zeeman effect . . . . . . . . . . . . . . . . . . . . 38

5.7 Microwave attenuation by the vacuum chamber . . . . . . . . . . . . . . . 39

5.8 Temperature dependence of the power-switching AOM $\ldots \ldots \ldots$. . . . 40

5.9 Line width versus intra-cavity power for delay $\tau_{6}=1010 \ldots 1210 \mu \mathrm{s} \ldots \ldots 43$

5.10 Comparison with former measurements . . . . . . . . . . . . . 45

$6.11 S-2 S$ resonance profiles of higher delays recorded with the high efficiency

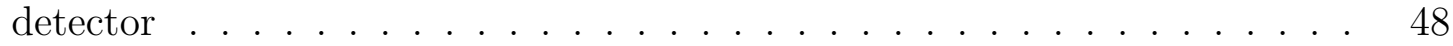

6.2 Comparison of old and new detector f . . . . . . . . . . . . . . 49

6.3 Comparison of May and November 2010 measurement . . . . . . . . . . . 52

6.4 Correction to the $1 S-2 S$ frequency for May and November 2010 . . . . . . 53

6.5 The SME coordinate system . . . . . . . . . . . . . . . . . . . . 54 
7.1 History of hydrogen spectroscopy . . . . . . . . . . . . . . . . . . 59

A.1 Setup for the comparison of two frequency combs . . . . . . . . . . . 62

B.1 Hydrogen absolute frequency data from the isotope shift measurement . . . 66

C.1 Analysis of artificial data for various simulation parameters . . . . . . . . 68

C.2 Power dependence of the simulated line width . . . . . . . . . . . . . . . 69

C.3 Linear ac Stark shift . . . . . . . . . . . . . . . . . . . . 70

C.4 Beam apparatus geometry used for simulation. . . . . . . . . . . . 71

D.1 Dye laser locking schematic . . . . . . . . . . . . . . . . . 73

D.2 Relative drift of dye laser and ECDL1 . . . . . . . . . . . . . . . . 75

D.3 Dye laser schematic . . . . . . . . . . . . . . . . . 76 


\section{List of Tables}

1.1 Summary of measurements . . . . . . . . . . . . . . . . . . . 3

2.1 Hyperfine splitting in atomic hydrogen, obtained from experiments . . . 8

3.1 FOM uncertainty budget . . . . . . . . . . . . . . . . . 17

4.1 Uncertainty budget for the isotope shift measurement . . . . . . . . . 26

5.1 Uncertainty budget for $f_{1 S-2 S}$ during the first measurement $\ldots \ldots \ldots 44$

6.1 Uncertainty budget for $f_{1 S-2 S}$ during the second measurement . . . . . . 51 


\section{Zusammenfassung}

Im Rahmen der vorliegenden Arbeit wurden drei Messungen am $1 S-2 S$-Übergang im atomaren Wasserstoff $(\mathrm{H})$ und Deuterium (D) durchgeführt. Der Übergang wurde zweiphotonisch, nahe $243 \mathrm{~nm}$, Doppler-frei an einem $5.8 \mathrm{~K}$ kalten Atomstrahl angeregt.

Die H/D-Isotopieverschiebung wurde zu [Parthey et at., PRL 104, 233001 (2010)]

$$
\Delta f_{\exp }=670994334606(15) \mathrm{Hz}
$$

bestimmt, eine zehnfache Verbesserung gegenüber der vormals besten Messung von Huber et al. [1]. Mit Hilfe des Vergleichs der theoretischen Isotopieverschiebung ohne führende Kernstruktur-Effekte, $\Delta f_{\text {th }}=670999566.90(66)(60) \mathrm{kHz}$ [2] können wir die RMS Ladungsradius-Differenz zwischen Deuteron und Proton, um einen Faktor zwei verbessert, bestätigen

$$
\left\langle r^{2}\right\rangle_{d}-\left\langle r^{2}\right\rangle_{p}=3.82007(65) \mathrm{fm}^{2},
$$

woraus sich der Deuteron-Strukturradius zu $r_{\mathrm{str}}=1.97507(78) \mathrm{fm}$ errechnen lässt.

Das Frequenzverhältnis des $1 S-2 S$-Übergangs in atomarem Wasserstoff zur Hyperfeinaufspaltung des Grundzustands von Cäsium wurde mit Hilfe der mobilen CäsiumFontänenuhr FOM gemessen [Parthey et al., PRL 107, 203001 (2011)]

$$
f_{1 S-2 S}=2466061413187035(10) \mathrm{Hz} .
$$

Dies bestätigt den bisherigen Wert von Fischer et al. [3], wobei wir die relative Unsicherheit um einen Faktor drei auf $4.2 \times 10^{-15}$ verringern konnten.

Eine zweite Messung der absoluten 1S-2S-Übergangsfrequenz im atomaren Wasserstoff stellt die erste Anwendung eines $900 \mathrm{~km}$ langen Faserlinks zwischen MPQ und der Physikalisch-Technische Bundesanstand (PTB) in Braunschweig dar. Der Link erlaubt die Kalibration des MPQ-Wasserstoff-Masers mit Hilfe der stationären Cäsium-Fontänenuhr CSF1 an der PTB. Das Ergebnis [Matveev et al., in Vorbereitung]

$$
f_{1 S-2 S}=2466061413187017(11) \mathrm{Hz}
$$

erlaubt die Einschränkung möglicher Elektron-Lorentz-Boost-verletzender Koeffizienten

$$
0.95 c_{(T X)}-0.29 c_{(T Y)}-0.08 c_{(T Z)}=(2.2 \pm 1.8) \times 10^{-11}
$$

innerhalb der minimalen Standardmodell-Erweiterungen. Ein möglicher Drift der starken Wechselwirkung lässt sich über die magnetischen Momente kompetitiv einschränken [4]

$$
\frac{\partial}{\partial t} \ln \frac{\mu_{\mathrm{Cs}}}{\mu_{B}}=-(3.0 \pm 1.2) \times 10^{-15} \mathrm{yr}^{-1} .
$$




\section{Abstract}

This Thesis reports on three measurements involving the $1 S-2 S$ transition in atomic hydrogen and deuterium conducted on a $5.8 \mathrm{~K}$ atomic beam. The transition is excited Dopplerfree via two counter-propagating photons near $243 \mathrm{~nm}$.

The H/D isotope shift has been determined as [Parthey et at., PRL 104, 233001 (2010)]

$$
\Delta f_{\exp }=670994334606(15) \mathrm{Hz}
$$

which is a ten-fold improvement over the previous best measurement by Huber et al. [1]. Comparing with the theoretical value for the isotope shift, excluding the leading nuclear size effect, $\Delta f_{\text {th }}=670999566.90(66)(60) \mathrm{kHz}[2]$ we confirm, twice more accurate, the rms charge radius difference of the deuteron and the proton as

$$
\left\langle r^{2}\right\rangle_{d}-\left\langle r^{2}\right\rangle_{p}=3.82007(65) \mathrm{fm}^{2}
$$

and the deuteron structure radius $r_{\mathrm{str}}=1.97507(78) \mathrm{fm}$.

The frequency ratio of the $1 S-2 S$ transition in atomic hydrogen to the cesium ground state hyperfine transition provided by the mobile cesium fountain clock FOM is measured to be [Parthey et al., PRL 107, 203001 (2011)]

$$
f_{1 S-2 S}=2466061413187035(10) \mathrm{Hz}
$$

which presents a fractional frequency uncertainty of $4.2 \times 10^{-15}$ and is a more than threefold improvement compared to the previous best measurement by Fischer et al. [3].

The second absolute frequency measurement of the $1 S-2 S$ transition in atomic hydrogen presents the first application of a $900 \mathrm{~km}$ fiber link between MPQ and PhysikalischTechniche Bundesanstand (PTB) in Braunschweig which we have used to calibrate the MPQ hydrogen maser with the stationary cesium fountain clock CSF1 at PTB. With the result of [Matveev et al., in preparation]

$$
f_{1 S-2 S}=2466061413187017(11) \mathrm{Hz}
$$

we can put a constraint on the electron Lorentz boost violating coefficients

$$
0.95 c_{(T X)}-0.29 c_{(T Y)}-0.08 c_{(T Z)}=(2.2 \pm 1.8) \times 10^{-11}
$$

within the framework of minimal standard model extensions. We limit a possible drift of the strong coupling constant through the ratio of magnetic moments at a competitive level [4]

$$
\frac{\partial}{\partial t} \ln \frac{\mu_{\mathrm{Cs}}}{\mu_{B}}=-(3.0 \pm 1.2) \times 10^{-15} \mathrm{yr}^{-1} .
$$


xiv 


\section{Chapter 1}

\section{Introduction}

There's a reason physicists are so successful with what they do, and that is they study the hydrogen atom and the helium ion and then they stop.

Richard Feynman

In nature, it is the simplest systems that allow us to find new, fundamental theories. If these simple systems are accessible to experiments as well, we can test and refine those theories to a high level of accuracy. Atomic hydrogen has been the Rosetta Stone [5] for atomic physics which led to the foundation of quantum mechanics and quantum electrodynamics.

When Fraunhofer discovered dark features in the optical spectrum of the sun in 1814, there where four hydrogen lines among the 51 dominant absorption lines he identified which later became known as Fraunhofer lines. Generalizing Balmer's work, Johannes Rydberg found the famous Rydberg formula

$$
\frac{1}{\lambda}=R_{\infty}\left(\frac{1}{n_{1}^{2}}-\frac{1}{n_{2}^{2}}\right)
$$

with the Rydberg constant $R_{\infty}=\frac{m_{e} e^{4}}{8 \varepsilon_{0}^{2} h^{3} c}$, where $m_{e}$ denotes the electron mass. Rydberg's key idea was to consider wave numbers rather than wavelengths.

When Bohr introduced his hydrogen model in 1916, he had to postulate the quantum nature by allowing the electron angular momentum to be integer multiples of $\hbar$ and requiring the electron to orbit the proton without losing energy via radiation. Sommerfeld extended Bohr's model to include relativistic effects.

With the arrival of the Dirac equation in 1928 the hydrogen atom seemed to be completely described and fully understood. It was only in 1947 that Lamb and Retherford found the $2 S$ Lamb shift lifting the degeneracy of states with the same principle quantum number $n$ and total orbital momentum $j$. Once again, hydrogen triggered the development of a new theory. Feynman, Schwinger and Tomonaga constituted quantum electrodynamics (QED), the electro-magnetic part of the standard model of particle physics. 
Since then, QED has been constantly tested. With the first observation of the $1 S-2 S$ transition in atomic hydrogen by Hänsch et al. in 1974, the era of laser spectroscopy of atomic hydrogen began. The $1 S-2 S$ transition with its natural line width of only $1.3 \mathrm{~Hz}$ offered a great potential for accuracy at that time - and still does. Also, an excitation with two photons is a twofold blessing. First, although the necessary $243 \mathrm{~nm}$ laser light lies in the ultraviolet, it can be generated by second-harmonic generation from continuous wave lasers with reasonable efficiency in commonly available beta barium borate (BBO) crystals. Second, the two-photon absorption allows to overcome the first order Doppler effect.

Interestingly, the $1 S-2 S$ spectroscopy in hydrogen is the single experiment with a fractional frequency uncertainty below $5 \times 10^{-15}$ without direct control of the species' motion via laser cooling. While this makes dealing with certain systematic shifts more challenging, it ultimately makes the experiment very simple and transparent.

Also, it should be pointed out that, for the first time in the long history of this experiment, the frequency measurement and the laser system are so robust and reliable that the work during the course of this Thesis could mostly concentrate on effects related to the hydrogen atoms themselves. Consequently, the majority of improvements presented in this Thesis relates to systematic effects concerned with the hydrogen beam and its interaction with the spectroscopy light.

The energy levels of hydrogen in SI units contain four parameters which need to be determined experimentally. These are the fine structure constant $\alpha$, the electron to proton mass ratio $m_{\mathrm{e}} / m_{\mathrm{p}}$, the Rydberg constant $R_{\infty}$ and the rms proton charge radius $\left\langle r_{p}^{2}\right\rangle$. While $\alpha$ is known very accurately from the electron $g-2$ experiment $[6,7]$ and $m_{\mathrm{e}} / m_{\mathrm{p}}$ has been measured precisely in Penning traps [8], the Rydberg constant and the proton charge radius can be determined from hydrogen spectroscopy. While the Rydberg constant is part of the Dirac energy and therefore enters the hydrogen energies as $R_{\infty} / n^{2}$, the proton charge radius is part of the finite-size correction to the Lamb shift which contributes as $L_{1 S} / n^{3}$. The different scaling of these two parameters allows their simultaneous determination by measuring different transition frequencies in hydrogen, preferably one between high- and one between low-lying states. With the measurement of more than two transitions - or by using $\left\langle r_{p}^{2}\right\rangle$ from electron scattering $[9,10]$ - one can start testing QED calculations.

In this context, the uniqueness of the $1 S-2 S$ transition poses a problem. With natural line widths in the megahertz range or, in the case of Rydberg states, high sensitivity to electric fields, every other transition than $1 S-2 S$ cannot be measured with similar accuracy. QED tests with hydrogen have been limited to the $10^{-12}$ level due to an insufficient knowledge of the proton charge radius. To overcome the insufficient knowledge of $\left\langle r_{p}^{2}\right\rangle$, Pohl et al. recently measured the Lamb shift in muonic hydrogen [11]. The scaling of the finite size effect with the orbiting particle's mass to the third power makes this effect much more dominant in muonic hydrogen. Thus, a more precise determination of $\left\langle r_{p}^{2}\right\rangle$ can be achieved with less experimental accuracy.

While the proton charge radius deduced from the measurement by Pohl et al. is ten times more accurate than the CODATA value [8] deduced from hydrogen and electron scattering, there exists a discrepancy of five CODATA standard deviations. The discrep- 
ancy has been dubbed proton size puzzle since it is not a priori clear whether there is a problem in the electron scattering data, ordinary hydrogen data or theory for either ordinary hydrogen or muonic hydrogen which is needed to extract the charge radius from the measured transition frequencies (see above). The work presented in this Thesis can contribute twofold. First, the $1 S-2 S$ transition of hydrogen serves as a cornerstone in the least squares adjustment leading to the CODATA proton charge radius extracted from hydrogen. Second, from the isotope shift presented here, we extract the charge radius difference of the deuteron and the proton. This will allow a compelling test when the same quantity will be extracted from muonic hydrogen/deuterium data which has been obtained [12].

Besides direct and indirect tests of QED, the $1 S-2 S$ transition has recently been used to test physics beyond the standard model. The measurements in 1999 and 2003 [13, 3] set a limit on the possible temporal variation of the fine structure constant $\alpha$. At that time, the value of $\dot{\alpha} / \alpha=(0.9 \pm 2.9) \times 10^{-15} \mathrm{yr}^{-1}$ was the most accurate limit in the current time epoch. This result has been outperformed by two orders of magnitude by comparison of two single ion atomic clocks at NIST in Boulder [14]. However, with the measurements presented in this Thesis we can set restrictions on the variation of the cesium nuclear magnetic moment to the Bohr magneton, effectively probing the strong interaction. The obtained results are competitive with the best constraints obtained from a single mercury ion [4].

There are other tests for which hydrogen's simple atomic structure outweighs the single ions' lower uncertainty. One of these tests arises from the standard model extensions (SME) introduced by Kostelecky [15]. These SME present a very systematic way to search for Lorentz and charge conjugation/parity / time reversal (CPT) symmetry violations. Using two measurements separated by half a year so that the Earth travels with opposite velocities in the sun-centered coordinate system, one can set limits on a linear combination of electron Lorentz boost violating parameters introduced within the SME.

A compelling but challenging test of CPT would be the comparison of the $1 S-2 S$ transitions in hydrogen and anti-hydrogen $[16,17,18,19]$.

Table 1.1: Summary of measurements

\begin{tabular}{l|l|l} 
Time & Measurement & \\
\hline Spring / Summer 2009 & $\begin{array}{l}\text { isotope shift } \\
\text { absolute frequency v. the mobile Cs fountain } \\
\text { clock FOM }\end{array}$ & Section 4 \\
Saytion 5 \\
October 2010 & $\begin{array}{l}\text { absolute frequency v. the stationary Cs foun- } \\
\text { tain clock CSF1 via the } 900 \mathrm{~km} \text { link }\end{array}$ & Section 5.3.3 \\
\hline November 2010 & Section 6
\end{tabular}




\section{Chapter 2}

\section{Theory}

This Chapter is intended to give a brief overview of the theory of the hydrogen atom. Anybody interested in the deep details of the theoretical description of the hydrogen atom shall be referred to Ref. [8], Sec. IV.1 and references therein which give a good summary

of the terms contributing to the hydrogen energy levels. The theory relevant for the determination of the deuteron-proton charge radius difference and that needed to restrict a possible electron Lorentz boost violation are discussed in Sections 4.5 and 6.5, respectively.

The biggest contribution to the hydrogen energy levels with the principle quantum number $n$ and electron angular momentum $j$ is given according to Dirac theory for an infinitely heavy nucleus and an orbiting electron of mass $m_{e}$, with the rest mass subtracted, as

$$
\begin{aligned}
E_{\mathrm{D}} & =m_{e} c^{2}[f(n, j)-1]=m_{e} c^{2}\left[\frac{1}{\sqrt{1+\epsilon(n, j)}}-1\right], \\
\epsilon(n, j) & =\frac{(Z \alpha)^{2}}{\left(n-j-\frac{1}{2}+\sqrt{\left(j+\frac{1}{2}\right)^{2}-(Z \alpha)^{2}}\right)^{2}} .
\end{aligned}
$$

The complete expression for the hydrogen energy levels in frequency units can be expressed as a sum of Dirac energy, the Lamb shift, and hyperfine splitting

$$
E_{H}=R_{\infty} c \frac{2 E_{n j}\left(n, j, \alpha, r_{N}\right)}{\alpha^{2} m_{e} c^{2}}+L\left(n, j, l, \alpha, r_{N}\right)+E_{\mathrm{HFS}}(n, j, l, F)
$$

with the nuclear to electron mass ratio $r_{N}=m_{e} / m_{N}$ which has been measured very accurately in Paul traps [8].

\subsection{Dirac Energy}

Excluding Lamb shift and hyperfine effects, but including reduced mass corrections, the bound state energy of the two-body Coulomb system is given by [20] 


$$
\begin{aligned}
& E_{n j}=m_{r}[f(n, j)-1]-\frac{m_{r}^{2}}{2\left(m_{\mathrm{e}}+m_{N}\right)}[f(n, j)-1]^{2} \\
& =m_{e} c^{2}\left\{\frac{1}{1+r_{N}} g(\epsilon(n, j))-\frac{r_{N}}{2\left(1+r_{N}\right)^{3}}[g(\epsilon(n, j))]^{2}\right\} .
\end{aligned}
$$

with

$$
g(\epsilon) \equiv \frac{1}{\sqrt{1+\epsilon}}-1=-\frac{\epsilon}{\sqrt{1+\epsilon}(1+\sqrt{1+\epsilon})},
$$

and the reduced mass $m_{r}$. This formula leaves the $(n, j)$ degeneracy of the levels intact. The representation on the right-hand side of Eq. (2.3) has the additional advantage that the Rydberg constant $R_{\infty}=m_{e} c \alpha^{2} /(2 h)$ can easily be factored out. The frequency corresponding to $E_{n j}$ is

$$
f_{n j}=\frac{2 E_{n j}}{\alpha^{2} m_{e} c^{2}} R_{\infty} c
$$

\subsection{Lamb Shift}

The real challenge in calculating the hydrogen spectrum today, lies in the calculation of the various relativistic and quantum electrodynamic (QED) calculations. Generally, these contributions are treated as a power series in $(Z \alpha)$. According to the widely used definition given in Eq. (2.2), all contributions to the hydrogen energy except for the Dirac energy (with reduced mass corrections) and the hyperfine splitting are called Lamb shift. The Lamb shift for ground state hydrogen is roughly $\mathcal{L}_{1 S} \approx 8.2 \mathrm{GHz}$. For higher levels, the Lamb shift decreases with $\mathcal{L}_{1 S} / n^{3}$.

These contributions, ordered by their numerical magnitude [8], are one-photon electron self energy, vacuum polarization, recoil corrections, radiative-recoil corrections, two-photon corrections, three-photon corrections, finite nuclear size effect, nuclear-size correction to self energy and vacuum polarization, and nucleus self energy.

The finite nuclear size effect is discussed in more detail in the context of the charge radius difference of proton and deuteron in Sec. 4.5 .

\subsection{Hyperfine Splitting}

The third term in Equation (2.2) is the hyperfine splitting (HFS) with its dependents on the total angular momentum $F$, the nuclear spin $I$, the electron's angular momentum $j$ and its orbital angular momentum $l[21]$ :

$$
E_{\mathrm{HFS}}(n, j, l, F)=\frac{\alpha^{2} g_{N} R_{\infty} c\left(1+\delta_{n}\right)}{n^{3} r_{N}} \frac{F(F+1)-I(I+1)-j(j+1)}{j(j+1)(2 l+1)}
$$




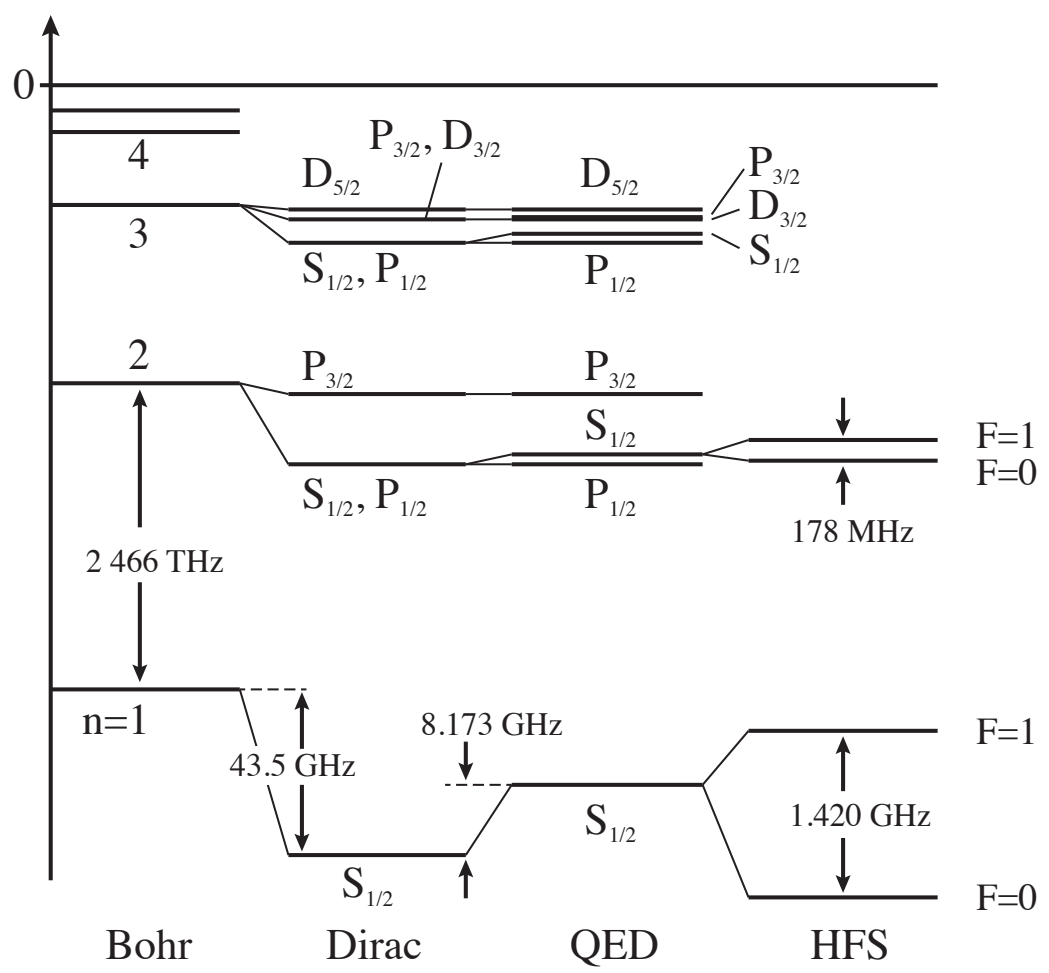

Figure 2.1: Energy levels in atomic hydrogen.

with the nuclear $g$-factor $g_{N}$ which relates the magnetic moment $\mu$ to the nuclear spin via $\mu=g_{N} \mu_{N} I . \delta_{n}$ is the small relativistic correction. The g-factor can be approximated as $g_{N}=2\left(1-\alpha^{2} /\left(3 n^{2}\right)\right.$ for $S$-states [21]. Thus, for transitions $\left(m_{F}= \pm 1\right) \rightarrow\left(m_{F^{\prime}}= \pm 1\right)$ we expect a linear dependence on magnetic fields.

The hyperfine structure is only well-known experimentally for the low-lying states with $n=1,2$. For higher lying states, the HFS is typically approximated from that of the low-lying states using Eq. (2.6). The HFS quickly becomes smaller for increasing principle quantum numbers due to the $n^{-3}$ dependence.

Accurate calculations of the hyperfine splittings are not available due to nuclear effects such as Zemach radius and polarizability [22]. However, in the linear combination $D_{21}=$ $8 f_{\mathrm{HFS}}(2 S)-f_{\mathrm{HFS}}(1 S)$ nuclear size effects cancel to large extend and accurate calculations allow testing theory [23, 24, 25] against experiment [26].

\subsubsection{Hyperfine Splitting in an External Magnetic Field}

In an external magnetic field the hyperfine levels of both the $1 S$ and $2 S$ level split. In the experiment, we apply an external magnetic field of $0.5 \mathrm{mT}$ to separate the hyperfine Zeeman levels with $m_{F}=0$ from those with $m_{F}= \pm 1$. We use the transitions from $F=1, m_{F}= \pm 1$ to $F=1, m_{F}= \pm 1$ for spectroscopy. They experience a Zeeman shift 
Table 2.1: Hyperfine splitting in atomic hydrogen, obtained from experiments.

\begin{tabular}{|c|c|c|}
\hline tral & $\begin{array}{r}\text { hydrogen } \\
F=1 \rightarrow F=1 \\
{[\mathrm{~Hz}]}\end{array}$ & $\begin{array}{r}\text { deuterium } \\
F=3 / 2 \rightarrow F=3 / 2 \\
{[\mathrm{~Hz}]}\end{array}$ \\
\hline $1 S$ & $1420405751.7667(10)[27]$ & $327384352.5222(17)[28]$ \\
\hline $2 S$ & $177556834.3(6.7)[26]$ & $40924454(7)[29]$ \\
\hline
\end{tabular}

of $\pm 360 \mathrm{~Hz} / \mathrm{mT}$. Working with an unpolarized atomic beam the shift of both transitions averages to zero. The hyperfine centroid frequency can be calculated from the $F=1, m_{F}=$ \pm 1 to $F=1, m_{F}= \pm 1$ transition by using the experimental results for the $1 S$ and $2 S$ hyperfine splitting $[27,26]$ (compare Tab. 2.1) by adding $\Delta f_{\mathrm{HFS}}^{\mathrm{H}}=+310712229.4(1.7) \mathrm{Hz}$.

In the case of deuterium the nuclear spin is $I=3 / 2$ and the hyperfine correction becomes $\Delta f_{\mathrm{HFS}}^{\mathrm{D}}=+71614974.6(1.8) \mathrm{Hz}$ (see Table 2.1) leading to a correction to the isotope shift of $\Delta f_{\mathrm{HFS}}^{\text {iso }}=+215225596.5(2.9) \mathrm{Hz}$.

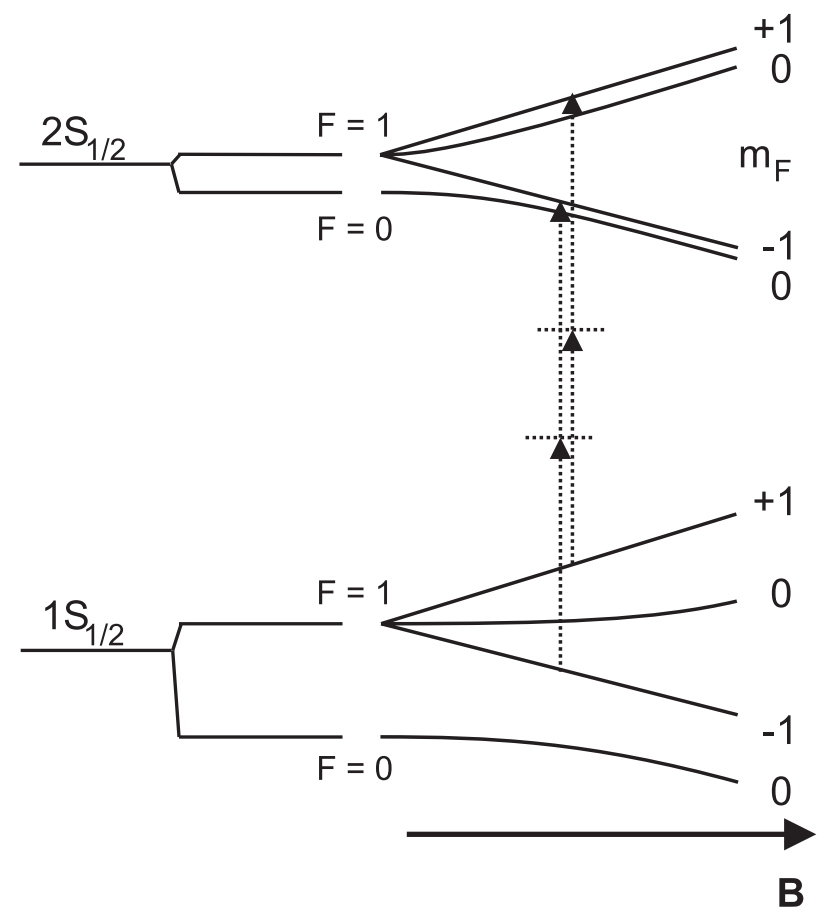

Figure 2.2: Hydrogen level scheme in an external magnetic field. From [30]. 


\section{Chapter 3}

\section{Experimental Procedure}

This Chapter presents an overview of the experimental techniques and the setup. We shall start with an overview of the beam apparatus. After introducing the laser systems used for the measurements presented in this Thesis, a summary of the frequency measurement, including the various frequency references employed, concludes this Chapter.

\subsection{Beam Apparatus}

Figure 3.1 shows the excitation and detection configuration which are similar to those used in previous experiments [3]. Molecular hydrogen and deuterium are purified in separate $\mathrm{Pd}$ filters and dissociated in a microwave discharge running in a sapphire tube at a pressure of $\sim 1 \mathrm{mbar}$ set by a needle valve. Passing a Teflon capillary of $0.7-1.0 \mathrm{~mm}$ in diameter directly after the discharge, the atoms are guided by Teflon tubing and thermalize at a copper nozzle ( $2 \mathrm{~mm}$ in diameter) cooled by a liquid helium flow cryostat. The maximum

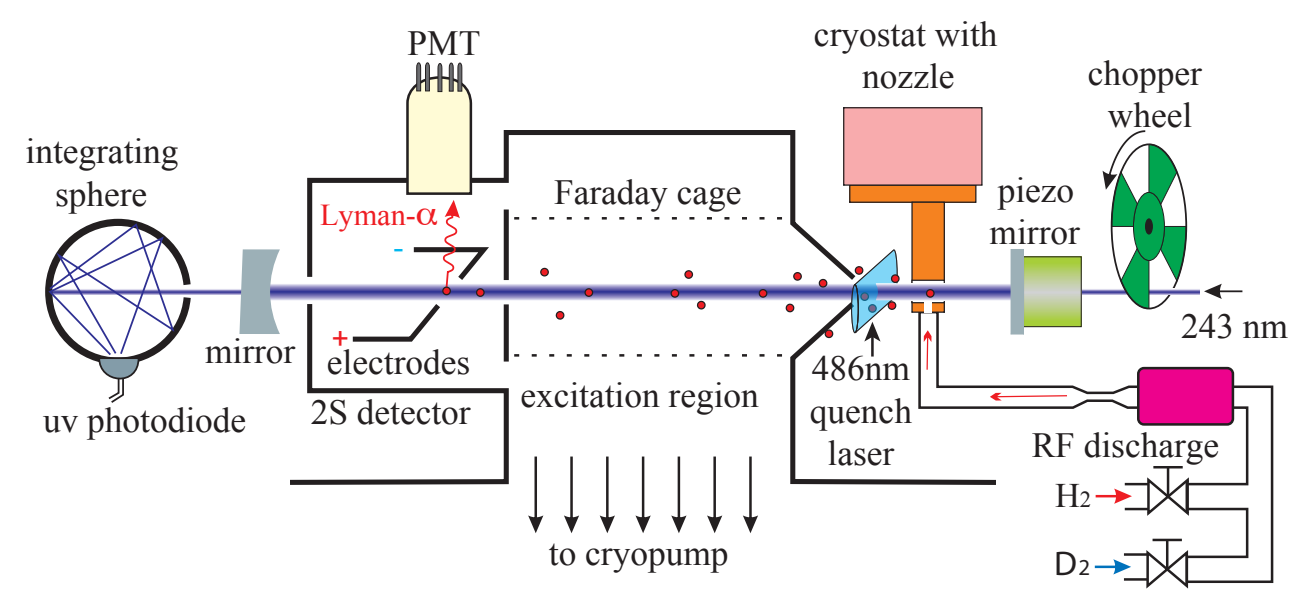

Figure 3.1: Beam apparatus for two-photon spectroscopy on the hydrogen / deuterium atomic beam. 
number of atoms is observed for nozzle temperatures of $5.8 \mathrm{~K}$ for $\mathrm{H}$ and $7.5 \mathrm{~K}$ for $\mathrm{D}$. The cold atomic beam is defined by two apertures at the beginning and the end of the excitation zone which is differentially pumped by a cryogenic pump and surrounded by a Faraday cage. All parts surrounding the excitation zone are covered with graphite to suppress stray electric fields. For detection we mix the excited atoms' $2 S$ state with the fast decaying $2 P$ states with a small electric field in front of a solar blind photo multiplier tube (PMT) that records the prompt emission of a $121 \mathrm{~nm}$ photon. A magnetic field of $0.5 \mathrm{mT}$ is applied to shift the magnetic field sensitive hyperfine components out of the laser tuning range.

Chopping the excitation light at $160 \mathrm{~Hz}$ with a $50 \%$ duty cycle allows to select the signal from the slow atoms: After the light has been turned off by the chopper we wait for a delay $\tau=10,210, \ldots 2210 \mu$ s to let the fast atoms escape the detector. Thus, lines recorded with higher delay $\tau$ exhibit a smaller second order Doppler effect at the expense of count rate. We use a multichannel scaler to simultaneously record 12 delayed lines [31].

\subsubsection{Quench Laser Beam near $486 \mathrm{~nm}$ at the Entrance of the Exci- tation Region}

After the isotope shift measurement but before the first absolute frequency measurement in May 2010, a quench beam at the entrance of the excitation region is introduced (see Figure 3.1). It resets atoms which are excited to the $2 S$ state within the nozzle region back to the ground state via the $2 S-4 P$ transition near $486 \mathrm{~nm}$. The reset minimizes the dc Stark shift due to possible stray fields within the nozzle region which is not shielded by a Faraday cage. Also, molecular hydrogen freezing at the nozzle might build up patch charges. In addition, the quench beam excludes the highest pressure region from contributing to the line shape, thus reducing the pressure shift (Section 5.3.7).

The beam is generated by focusing the dye laser (3.2.1) with a cylindrical lense. The generated "razor blade" has a waist of $200 \mu \mathrm{m}$ by $2 \mathrm{~cm}$ and a power of around $15 \mathrm{~mW}$. During the experiments, the power is re-adjusted via a half-wave plate in front of a polarizer at regular time intervals. The beam can be steered by a mirror located outside of the vacuum chamber. Additional mirrors inside the chamber guide the beam from its dedicated viewport through a hole in the aluminum frame supporting the enhancement cavity. Finally, the quench beam is dumped on the wall of the vacuum tubing leading to the turbo-molecular pump.

\subsubsection{High-Efficiency Lyman- $\alpha$ Detector}

The new high-efficiency Lyman- $\alpha$ detector is shown in Figure 3.2 and was introduced for the second absolute frequency measurement in November 2010. It has been redesigned for higher quantum efficiency compared to previous measurements [3, 13, 32]. Instead of detecting the emitted $121 \mathrm{~nm}$ Lyman- $\alpha$ photons directly, we detect photo-electrons created when the photons hit the graphite coating covering the $2 S$ detector inner walls. The photo-electrons are collected by a channeltron whose front face is biased at $+270 \mathrm{~V}$. The 




Figure 3.2: Schematic of the high-efficiency Lyman- $\alpha$ detector. Photo-electrons are collected by a channeltron leading to a large detection solid angle.

atomic beam is shielded from this voltage by a second Faraday cage to maintain the well localized quenching point created by the quench electrodes. The photo-electron detector increases the detection efficiency by more than an order of magnitude due to an increase of the solid angle compared to the previously used photo multiplier tube configuration (see Section 6.2.2).

\subsubsection{Enhancement-Cavity Transmission}

The chopper for the excitation light is set up in a way, that the bright and dark periods are $3.125 \mathrm{~ms}$ each $(160 \mathrm{~Hz})$. In Figure 3.3 one can see, that the enhancement cavity stabilization oscillates for approximately $1.5 \mathrm{~ms}$. Atoms contributing to very high delays $\tau>1600 \mu \mathrm{s}$ are more likely to experience this transient oscillation resulting in an inaccurate knowledge of the excitation power. It is thus better do discard these atoms from the analysis. If one wants to work with these atoms in the future, the chopping frequency should be reduced.

\subsection{Laser System}

In our lab, we operate three laser systems related to the $1 S-2 S$ transition in atomic hydrogen. They shall be described in this section along with their individual reference cavities.

\subsubsection{Dye Laser}

The second harmonic of a dye laser at $486 \mathrm{~nm}$ has been used as main spectroscopy laser for experiments (e.g. Refs.[13, 3, 29, 26]) on the $1 S-2 S$ transition prior to the work presented in this Thesis. Its frequency is phase stabilized to a spring-suspended ULE cavity in 

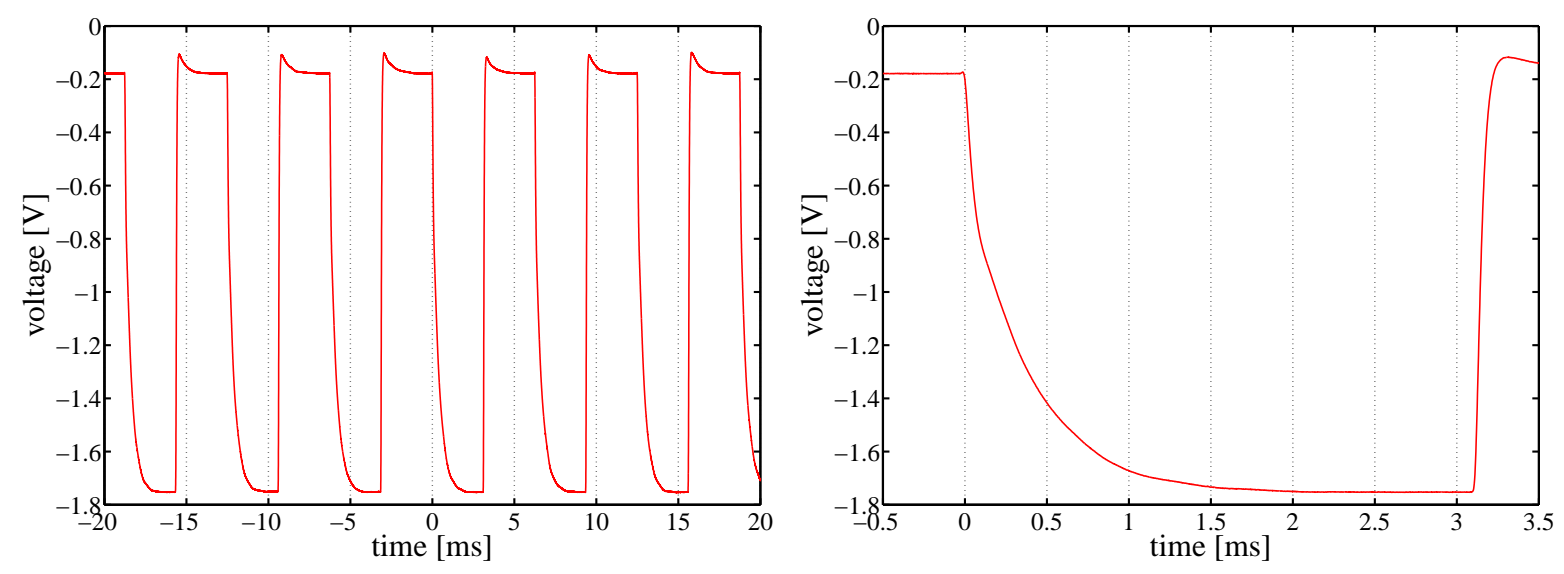

Figure 3.3: Shown is the measured transmission of the enhancement cavity. The vertical axis is inverted, so more negative values correspond to a higher transmission. The depicted trace is an average of 2000 chopper cycles. The lock takes about $1.5 \mathrm{~ms}$ to settle.

horizontal configuration (FP3 in Figure 3.5). The laser has an irregular frequency drift of less than $1 \mathrm{~Hz} / \mathrm{s}$ and a line width of $60 \mathrm{~Hz}$ measured over $0.2 \mathrm{~s}$ [33].

The frequency doubled dye laser frequency can be tuned to the $1 S-2 S$ transition in atomic hydrogen and well as deuterium and its fundamental frequency can be tuned in resonance with the $2 S-4 P$ transition by choosing the right cavity mode of FP3.

\subsubsection{Extended-Cavity Diode Laser ECDL1}

The main spectroscopy laser system (Figure 3.4) is a frequency quadrupled master oscillator power amplifier seeded by a $20 \mathrm{~cm}$ long extended cavity diode laser at $972 \mathrm{~nm}$ (ECDL1) which is tuned to the eighth subharmonic of the $1 S-2 S$ transition. The laser system is discussed in great detail in Ref. [34]. The laser frequency is stabilized to a transmission peak of a vibrationally- and thermally-isolated high finesse cavity (FP1) made from ultralow expansion glass (ULE). The cavity is stabilized at its zero expansion temperature $T_{\mathrm{c}}$ which greatly reduces its temperature sensitivity [35]. The laser frequency can be tuned with respect to the cavity using an acousto-optic modulator (AOM 1).

Orienting the cavity in an upright position mounted on the gravitational mid-plane minimizes the sensitivity to vertical vibrations, e.g. for an acceleration pointing downwards, the lower part is compressed the same way the upper part is extended resulting in no net length change [36]. The laser is continuously kept in lock over each measurement day showing an almost linear frequency drift of $+50 \mathrm{mHz} / \mathrm{s}\left(+1.6 \times 10^{-16} \mathrm{~s}^{-1}\right)$. If the laser lock to the cavity is lost, the cavity's drift changes after re-locking due to the temperature change caused by the laser radiation. Thus, if the cavity lock is lost during a measurement, 


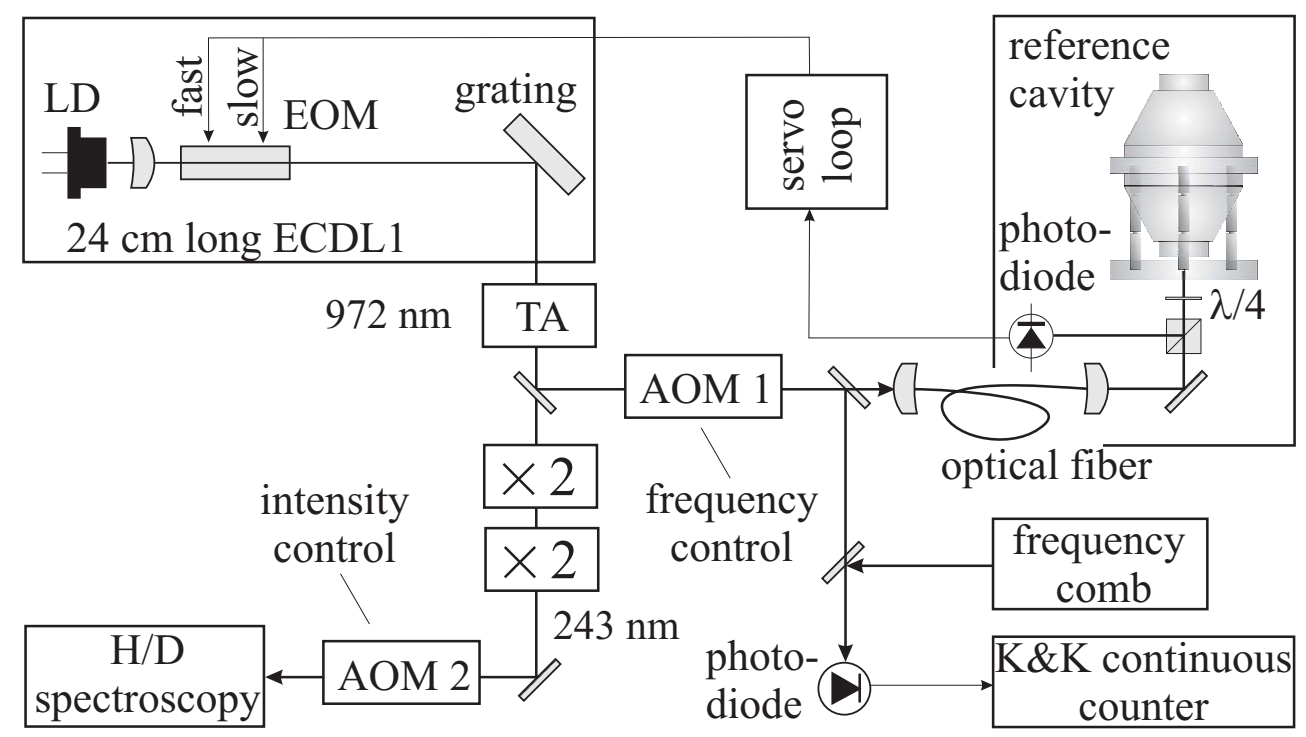

Figure 3.4: Schematic of the extended-cavity diode laser system. This setup was used for all $1 S-2 S$ spectroscopy measurements except for the first nine days of the isotope shift measurement (see also Figure 3.5). $\times 2$-second harmonic generation stage, AOM - acousto-optic modulator, ECDL - extended cavity diode laser, EOM electro-optic modulator, $\mathrm{K} \& \mathrm{~K}$ - Klische and Kramer counters operated in $\Lambda$ mode, TA - tapered amplifier.

we wait for 20 min to let the cavity re-thermalize.

The spectral line width of the laser after the tapered amplifier was measured to be less than $0.5 \mathrm{~Hz}$ and its relative frequency instability reaches $4 \times 10^{-15}$ in $10^{3} \mathrm{~s}$. To scan over the atomic transition, the frequency of ECDL1 is shifted in steps by a double pass acousto-optic modulator (AOM) installed between FP1 and ECDL1.

ECDL1 can also be tuned to the $1 S-2 S$ transition in atomic deuterium by changing the stabilization to a different transmission peak of FP1 and operating the corresponding AOM in a single pass configuration.

\subsubsection{Extended-Cavity Diode Laser ECDL2}

The second diode laser ECDL2 near $972 \mathrm{~nm}$ which is continuously phase-stabilized to the FP2 ULE cavity (Figure 3.5) cannot be efficiently quadrupled for $1 S-2 S$ spectroscopy due to insufficient suppression of its noise pedestal [34].

Instead, it has been used for auxiliary tasks during the course of this Thesis. For the isotope shift measurement, ECDL2's frequency was single-pass doubled to $486 \mathrm{~nm}$ and used as a transfer oscillator to connect the dye laser to the frequency comb. During the measurement of the velocity distribution for the $1 S-2 S$ absolute frequency measurement, ECDL2 was used as seed laser for a tapered amplifier whose output was frequency doubled 


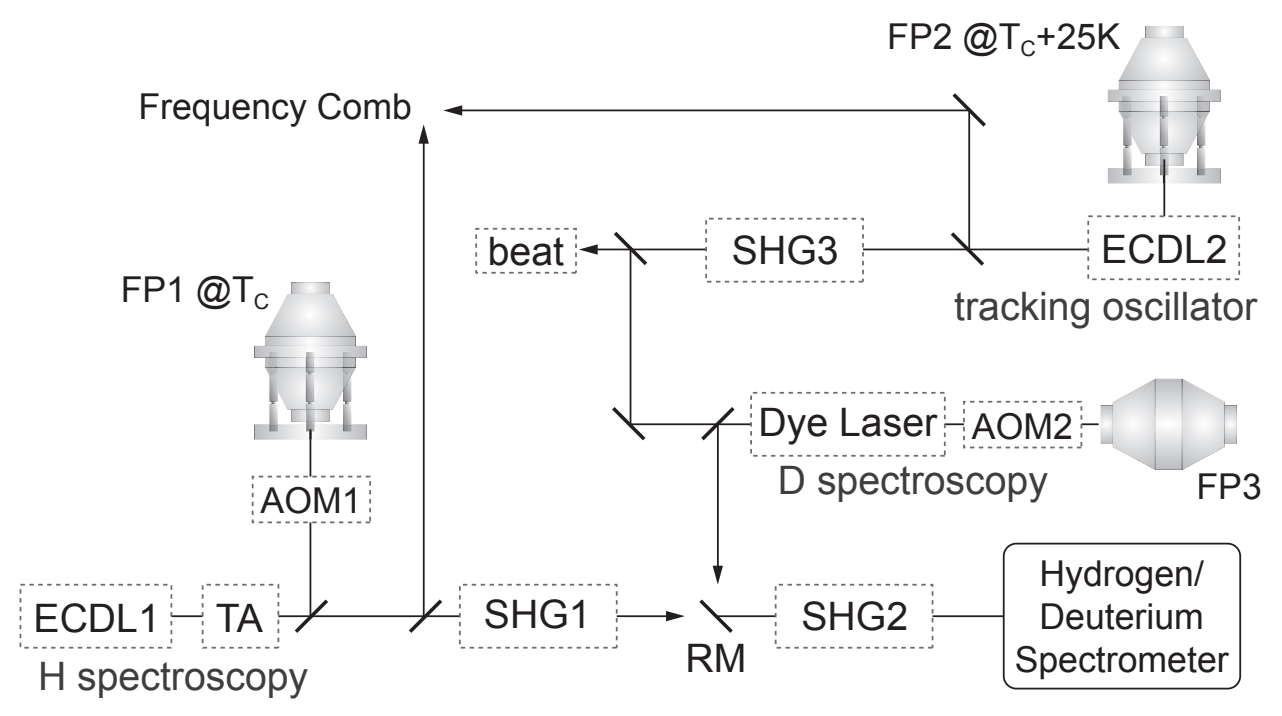

Figure 3.5: Schematic of the laser system used during the first nine days of the isotope shift measurement. AOM-acousto-optic modulator, ECDL-extended cavity diode laser, FP-Fabry-Perot cavity, $T_{\mathrm{c}}$ - zero expansion temperature, $\mathrm{SHG}-$ second harmonic generation stage, TA - tapered amplifier, RM-removable mirror.

in an enhancement cavity to create the $486 \mathrm{~nm}$ radiation necessary to excite the $2 S-4 P$ transition.

Between the two isotope shift measurement runs in Spring 2009 and late Summer 2009, the temperature stabilization of FP2 has been upgraded to support stabilization of FP2 at its zero expansion temperature $T_{\mathrm{c}}$. Since the upgrade, FP2's stability is comparable to that of FP1.

\subsection{Frequency Measurement}

The Allan deviations [37] for most devices envolved in the optical frequency measurement are presented in Fig. 3.6. One can identify the averaging times at which one should change from one reference to the other. For the case of an absolute frequency measurement with the mobile cesium fountain clock FOM (compare Section 3.3.3) as primary reference, for the first 700 s FP1 should be used, from 700 s to 20000 s one should rely on the maser before using FOM for the long term stability. From the comparison of two combs (Appendix A) one can see, that the comb systems do not contribute to the measurements instability at any time (pink curve in Fig. 3.6). 


\subsubsection{Frequency Comb System}

In our lab we operate an erbium-doped fiber frequency comb. The system was developed by Menlo Systems and assembled by Tobias Wilken. The femto-second oscillator runs near $1500 \mathrm{~nm}$. To generate light at $972 \mathrm{~nm}$ needed for the beat note with ECDL1/2, light near $1944 \mathrm{~nm}$ is generated from the comb's output using a nonlinear fiber. It is then single-pass frequency-doubled in free space. The comb runs at a repetition rate $f_{\text {rep }}=250 \mathrm{MHz}$. The carrier envelope phase $f_{\mathrm{CEO}}$ was changed several times during the work of this Thesis. For the absolute frequency measurement it was phase-locked to $30 \mathrm{MHz}$. All frequencies are treated redundantly as explained in Appendix A.2.

\subsubsection{Maser and Radio Frequency Multiplication}

At MPQ we operate an active hydrogen maser (Kvarz CH1-75A) as frequency reference. Most importantly, the maser is used to phase-lock the frequency comb's repetition rate $f_{\text {rep }}=250 \mathrm{MHz}$ at it's forth harmonic at $1 \mathrm{GHz}$. For that, the $10 \mathrm{MHz}$ output is multiplied up to $1 \mathrm{GHz}$ with three, individually temperature stabilized, radio frequency multipliers $(\times 4, \times 5, \times 5)$ from Wenzel, Austin, USA. A test of the radio frequency branch of the comb can be found in Appendix A.



Figure 3.6: Allan deviations for the frequency components present in our experiments. For traces involving a cavity, the linear drift has been subtracted. 


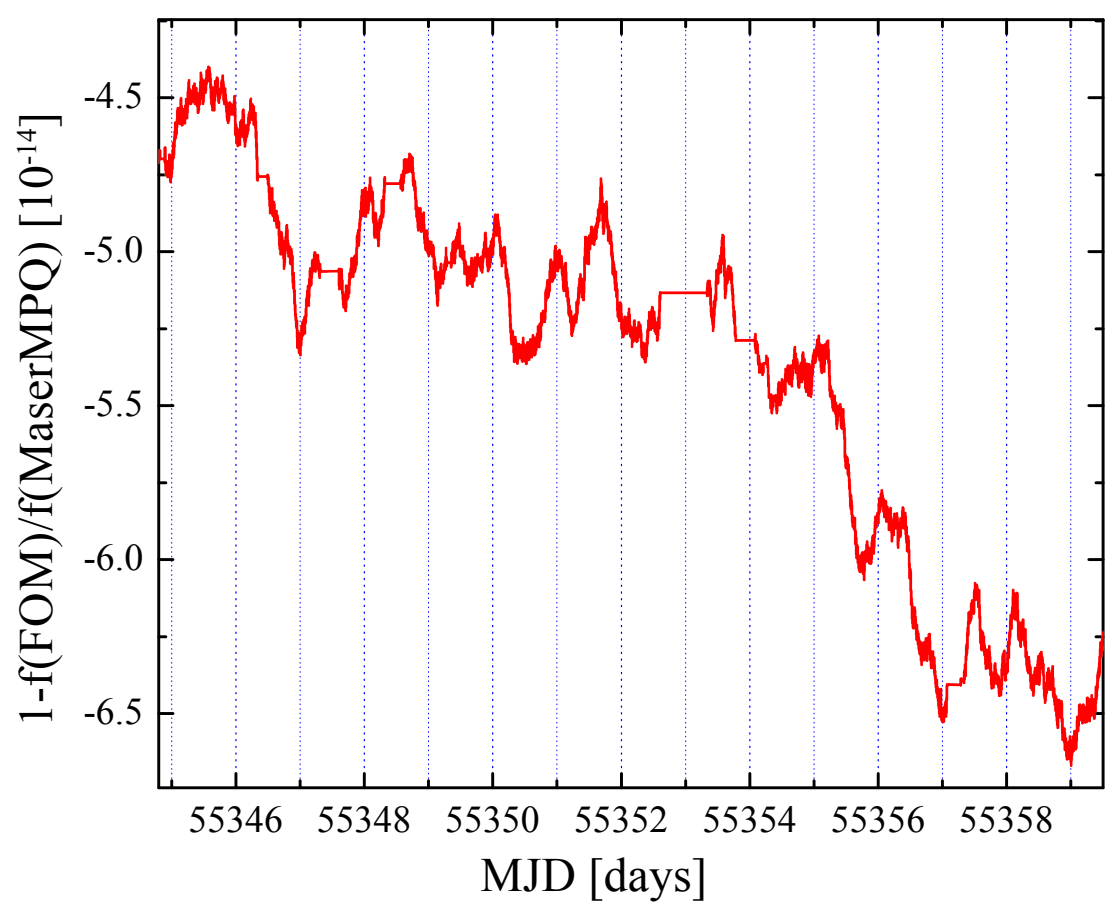

Figure 3.7: Fractional frequency difference of the MPQ maser versus FOM. The red line shows the $20000 \mathrm{~s}$ average used for calibration. Regions with maser drifts with up to $10^{-14} \mathrm{~d}^{-1}$ are identifiable (e.g. MJD 55346). MJD-Modified Julian Date.

The maser serves as reference for all synthesizers and counters used in the experiment.

\subsubsection{Maser Calibration}

\section{Global Positioning System}

We continuously monitor the maser operation via GPS. Although a statistical analysis of this calibration suggests a fractional frequency inaccuracy of $4 \times 10^{-15}$, the GPS calibration with our maser can only be trusted on the $10^{-14}$ level: One has to average the GPS signal for about $10^{5}$ s to reach a stability of $4 \times 10^{-15}$ (Fig. 3.6). However, from Fig. 3.7 we can see that the maser can drift up to $10^{-14}$ within this averaging time.

\section{Mobile Cesium Fountain FOM}

For the absolute frequency measurement in May 2010 (Section 5) we invited a team from the LNE-SYRTE, the French standard institute, to visit our group with their mobile atomic cesium fountain clock FOM [38]. For this measurement, the hydrogen $1 S-2 S$ frequency was linked to the maser as usual and then the maser was calibrated using FOM. For this calibration, the maser's $100 \mathrm{MHz}$ output was multiplied and offset to $9.1926 \mathrm{GHz}$ for 
Table 3.1: Uncertainty budget for FOM during its operation at MPQ [39].

\begin{tabular}{c|c|c} 
& correction $\left[10^{-16}\right]$ & uncertainty $\left[10^{-16}\right]$ \\
\hline quadratic Zeeman effect & -295.9 & 1.2 \\
\hline black body radiation & 165.8 & 0.6 \\
\hline cold collisions and cavity pulling & 25.1 & 5.0 \\
\hline $\begin{array}{c}\text { microwave power dependence: } \\
\text { first order Doppler and Microwave } \\
\text { spectral purity and leakage }\end{array}$ & 0 & 6 \\
\hline Ramsey and Rabi pulling & 0 & $<0.1$ \\
\hline microwave recoil & 0 & $<1.4$ \\
\hline second order Doppler effect & 0 & $<0.1$ \\
\hline background gas collisions & 0 & 8.1
\end{tabular}

probing the cesium ground state hyperfine splitting (clock transition). The result of this comparison can be seen in Fig. 3.7.

The fractional frequency uncertainty of FOM during its operation at MPQ [39] has been evaluated to be

$$
\sigma_{\mathrm{FOM}} / f_{\mathrm{FOM}}=8.1 \times 10^{-16} .
$$

The individual uncertainty contributions and corrections are summarized in Tab. 3.1. The cesium fountain clock probes a magnetic sensitive transition in an externally applied, magnetic bias field. Consequently, the quadratic Zeeman shift contributes the largest correction.

\section{Optical Fiber Link and CSF1}

The frequency measurement for the November 2010 measurement (Section 6) is schematically depicted in Fig. 3.8. The frequency of FP1 is referenced to the active hydrogen maser via the femtosecond erbium-doped fiber frequency comb. The maser is calibrated using the stationary cesium atomic fountain clock CSF1 [40, 41] located at PhysikalischTechnische Bundesanstalt (PTB) in Braunschweig. To compare the signal over a distance of $700 \mathrm{~km}$ between MPQ in Garching and PTB, we use a $900 \mathrm{~km}$ long actively stabilized fiber link [42]. The link allows to measure an ultra-stable transfer laser running at the telecom wavelength $1542 \mathrm{~nm}$ simultaneously at MPQ against the maser and at PTB against CSF1 with the help of a frequency comb and another ultra-stable laser at PTB. Thus, the two frequency references can be directly compared. The link's fractional frequency stability of $3 \times 10^{-14} \mathrm{~s} \tau^{-1}$ - with the averaging time $\tau$ - exceeds the stability needed for such a radio frequency reference comparison [43].

The correction and uncertainty of CSF1 during the November 2010 measurement was 
evaluated as

$$
\Delta f_{\mathrm{CSF} 1}=+370.0(7.6) \times 10^{-16} .
$$

The differential gravitational red shift between CSF1 and the hydrogen experiment can be calculated from the individual red shifts with respect to the geoid

$$
\Delta f_{\text {red shift }}=f_{\text {red shift }}^{\mathrm{MPQ}}-f_{\text {red shift }}^{\mathrm{PTB}}=(-52.7+8.6) \times 10^{-15}=44.1 \times 10^{-15} .
$$

with an uncertainty of $0.077 \times 10^{-15}$, corresponding to an uncertainty in the height difference of the two experiments of $70 \mathrm{~cm}$.

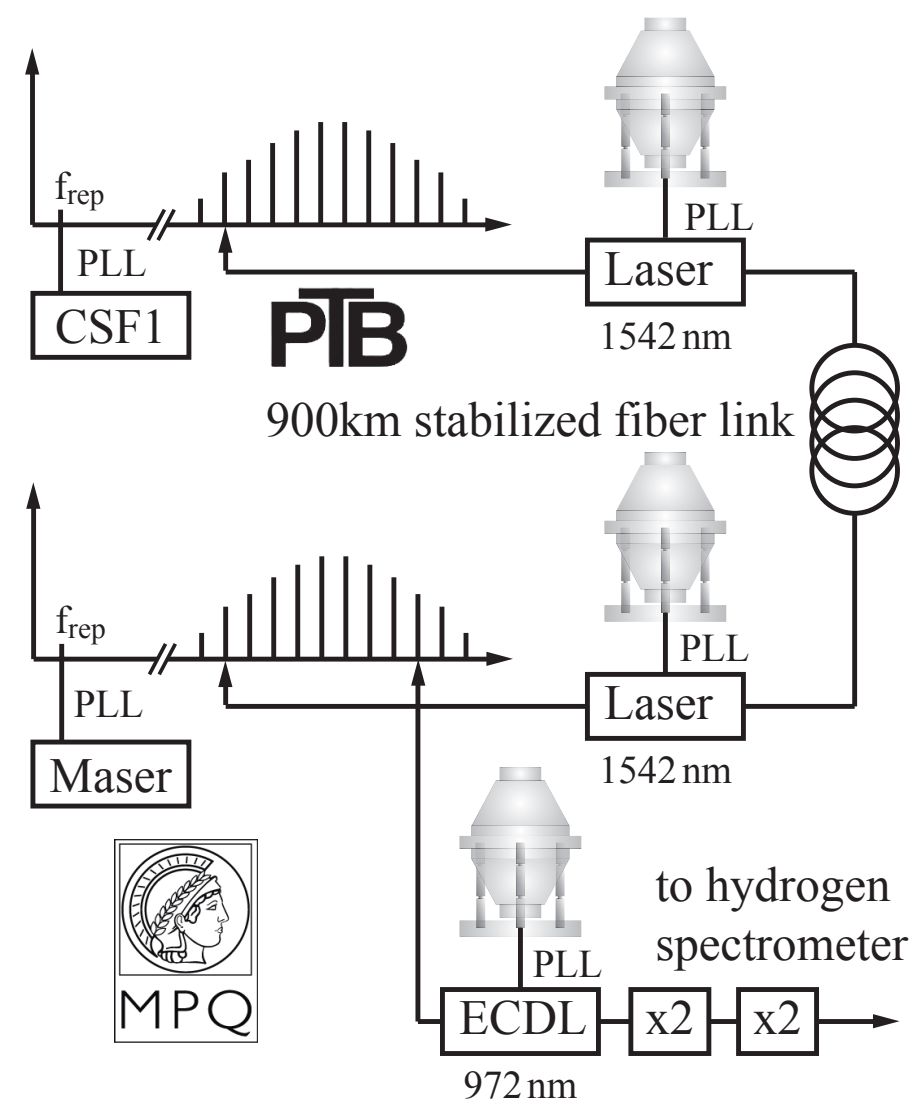

Figure 3.8: Schematic of the frequency measurement using CSF1 and the optical fiber link. PLL - phase lock loop, $f_{\text {rep }}$ - repetition rate, ECDL - extended cavity diode laser used for the hydrogen spectroscopy, CSF1 - cesium fountain clock. 


\section{Chapter 4}

\section{The 1S-2S Hydrogen / Deuterium Isotope Shift}

\subsection{Introduction}

With the implementation of the new diode laser based laser system (see Section 3.2.2) there was hope that the excessive data scatter of the previous two absolute frequency measurements $[13,3]$ might be overcome (Appendix D). Thus, in early 2008, we decided to re-measure the absolute frequency with the new laser system and the old beam apparatus. For this big goal, the isotope shift measurement presented a perfect preparation: Such a differential frequency measurement is easier in several ways: First, for the frequency comparison over time we only need a stable but not accurate frequency reference, so the active hydrogen maser is perfectly suited. Second, many systematic effects such as the second order Doppler effect or the ac Stark shift are common mode to both isotopes and cancel to large extend.

On the other hand, the data taken for hydrogen can be evaluated independently, referenced to the maser with GPS. In that way, an absolute frequency with an accuracy in the few $10^{-14}$ can be achieved. This accuracy was sufficient to conclude that the excessive day-to-day scatter had been removed with the new laser system and that an absolute frequency measurement was feasible (see Appendix B).

Today, precision atomic spectroscopy is the most powerful tool to study the root mean square charge radius of simple nuclei $[44,45,46,47,48]$. The simplest compound nucleus, the deuteron, is of great interest to test nuclear few body physics. Historically, its charge radius has been determined by scattering experiments [49], until precision measurements of the hydrogen-deuterium (H-D) $1 S-2 S$ isotope shift became competitive $[50,51,1]$. Although the nuclear size corrections to the electronic energy levels are as small as $10^{-10}$ in relative units, they contribute with the largest uncertainty to the theoretical description within the framework of quantum electrodynamics (QED). Therefore, the charge radii (or differences) may be extracted from absolute frequency measurements (or isotope shifts). These determinations rely on the correctness of QED calculations. 
To allow the determination of the charge radii and simultaneously verify the predictions of QED, a measurement of the $2 \mathrm{~S}$ Lamb shift in muonic hydrogen $(\mu \mathrm{p})$ has been conducted recently [11]. With the same nucleus as in ordinary hydrogen but with an orbiting lepton that is $\sim 200$ times heavier, the finite size correction in this system is $\sim 200^{3}$ larger. Previous results obtained from $\mathrm{H}$ spectroscopy, as expressed by the CODATA [8] value for the proton mean square charge radius $\left\langle r^{2}\right\rangle_{p}$, differ significantly from the recent value obtained from $\mu \mathrm{p}$. This provides motivation for an improved measurement of the $\mathrm{H}-\mathrm{D}$ isotope shift. Furthermore, it stimulates theoretical discussions about the interpretation of the charge radius $[52,53]$, the consistency of the electron scattering data with spectroscopy experiments, and calculations of the deuteron polarizability $[54,55]$.

\subsection{Experimental Procedure}

For the H-D isotope shift based on two-photon spectroscopy of the $1 S-2 S$ transition two data runs in March/April 2009 (first run) and August 2009 to January 2010 (second run) were recorded. A diode laser and a dye laser (see Section 3.1) were used for H and D in the first run, respectively (see Figure 3.5), whereas in the second run, both isotopes were excited by the diode laser system (see Figure 3.4).

\subsubsection{Differential Frequency Measurement with the Maser as Fly- wheel}

The actual laser frequency is measured by an Er-doped fiber frequency comb (see Section 3.3.1) referenced to an active, Global Positioning System (GPS) disciplined hydrogen maser. According to its specification, the fractional frequency instability of the maser is lower than $2 \times 10^{-15}$ on the time interval from $10^{3}$ to $10^{5} \mathrm{~s}$. GPS calibration provides a frequency inaccuracy on the order of $10^{-14}$. For our differential frequency measurement the frequency stability of the reference is much more important than its accuracy. The lowest uncertainty for $\Delta f_{\exp }$ is reached when $f_{1 S-2 S}^{D}$ and $f_{1 S-2 S}^{H}$ are measured within one day.

\subsubsection{First Measurement Run with Dye Laser and ECDL1}

During the first run, the $1 S-2 S$ transition in atomic deuterium was excited with the second harmonic of the dye laser operated near $486 \mathrm{~nm}$ locked to a spring-suspended ULE cavity in horizontal configuration (FP3). The second diode laser ECDL2, which is continuously locked to the FP2 ULE cavity, was used as a transfer oscillator between the frequency comb and the dye laser (compare Figure 3.5). Hydrogen was excited with the forth harmonic of ECDL1. In the first run all beat notes were counted using HP/ Agilent 53131A counters. Compared to Klische \& Kramer counters, the HP model have the disadvantage of random dead times. To make these counters record simultaniously, as needed for cycle 
slip detection [56], a hard reset is required for each frequency point. Due to internal delays, this leads to a total recording time of almost $3 \mathrm{~s}$ for a single $1 \mathrm{~s}$ gate time.

\subsubsection{Second Measurement Run with ECDL1 exclusively}

In the second run we use ECDL1 as the spectroscopy laser for both isotopes (see Figure 3.4). This is advantageous since its almost perfectly linear drift allows us to fit its beat frequency with the comb by a straight line. This allows a more accurate averaging of the maser noise as compared to the segment wise parabolic fit used to approximate the nonlinear drift of FP2. ECDL2 with FP2 is only used to monitor the correct operation of ECDL1 through a transfer beat via the frequency comb [57]. Instead of measuring the actual laser frequency we measure the frequency of the light coupled to the cavities, decoupling the frequency determination from the spectroscopy (compare Figure 3.4). In the second run, all frequencies are counted with Klische \& Kramer FX-80 continuous counters. The beam apparatus is described in Section 3.1.

\subsubsection{Data Taking Protocol}

On each measurement day we randomly pick one isotope to start with and record 30 to $1001 S-2 S$ spectra during less than four hours. Then, we promptly switch to the other isotope and record a similar number of lines. Since this procedure requires to break the lock of ECDL1 to the cavity, we wait for 20 min after the lock to the cavity is restored. During this time the cavity heats up due to the laser radiation and reaches a steady state with its well defined drift rate.

To scan over the line the laser probes the transition at optical frequencies in a random order to avoid possible systematics associated with the scan direction. At each laser frequency we alternate between two laser power levels by using a double pass AOM operating in zeroth order. The two simultaneously recorded lines allow us to cancel the ac Stark shift (Section 4.3.3). In total we recorded 3770 lines during 35 days of measurement.

\subsection{Systematic Uncertainties}

We now consider the major systematic contributions and error budget for our setup. A lot of systematic effects are common to both isotopes and thus, cancel to a large amount.

\subsubsection{Second Order Doppler effect}

The recorded time-delayed spectra at each delay $\tau$ are fitted by a Lorentzian, in order to assess the second order Doppler effect. For small $\tau$ the line is strongly asymmetric but the asymmetry gradually vanishes for larger delays (see Figure 5.2) as the velocity distribution of the contributing atoms becomes more narrow. For the final analysis, we only use delay $\tau=1410 \mu \mathrm{s}$ as it combines a small Doppler effect while still providing a 

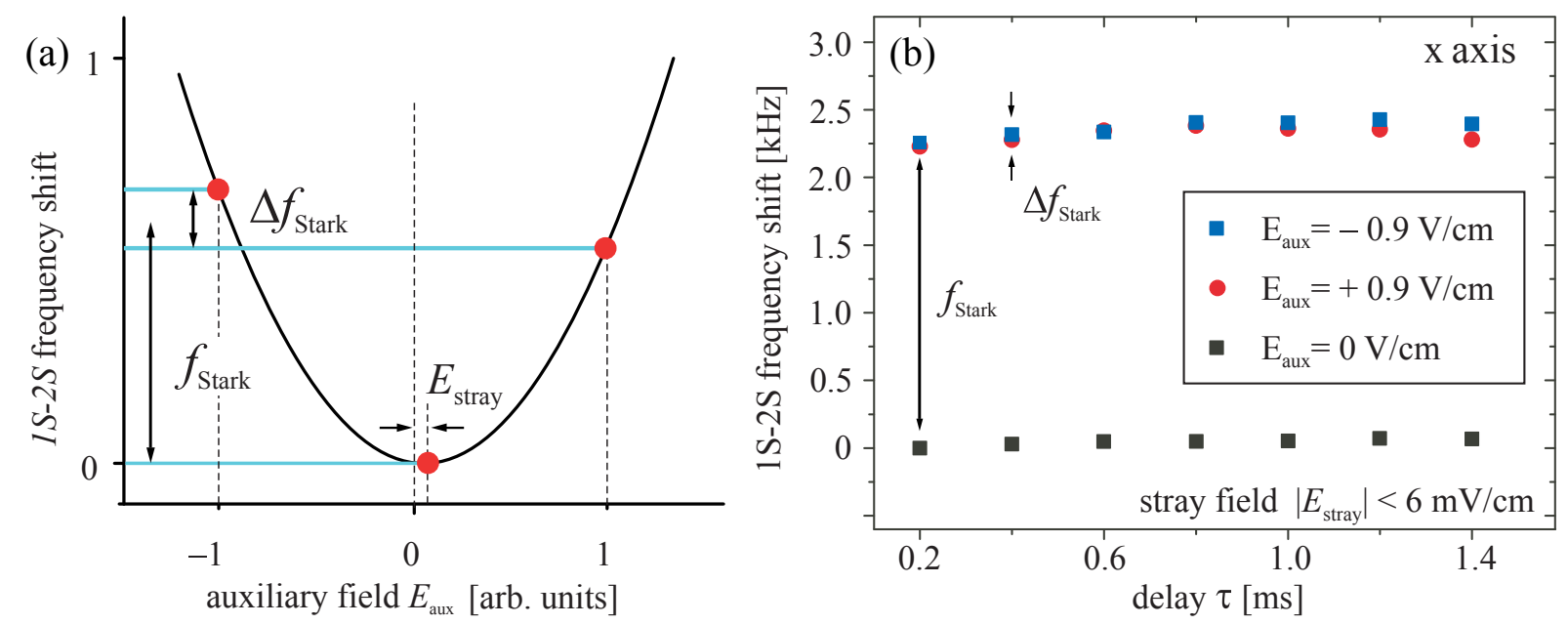

Figure 4.1: (a) Characterization of the stray fields in the excitation region by help of an auxiliary electric field $\pm E_{\text {aux }}$ applied by a plane capacitor field along one of the axes $x, y, z$. Measuring the transition frequency for an auxiliary field $\pm E_{\text {aux }}$ and zero auxiliary field, we can fit a three-parameter parabola to the data. The deviation of the center of the parabola from the point recorded at zero auxiliary field then is a measure of the dc Stark shift due to stray fields. (b) The probing of the stray fields along the $x$ axis in our experimental setup is illustrated.

strong signal with good statistics. A Monte Carlo (MC) simulation [58] shows that for delay $1410 \mu \mathrm{s}$, the frequency shift due to the second order Doppler effect is on the order of $-20 \mathrm{~Hz}$ and is largely independent of the particle mass $m$ and the beam temperature $T$ and thus cancels when the H-D frequencies are subtracted. As has been observed before [31], incomplete thermalization, geometry and other effects may alter the velocity distribution. These effects may, moreover, depend on the isotope. In order to cover conceivable extreme deviations from the Maxwellian case, we have performed MC simulations with distributions $f(v) \propto v^{2 \ldots 5} \exp \left(\frac{m v^{2}}{2 k_{\mathrm{B}} T}\right)$. Here, $v$ is the particle velocity and $k_{\mathrm{B}}$ is Boltzmann's constant. We find an upper bound of $6 \mathrm{~Hz}$ for the velocity distribution dependent correction. We use this value to estimate the uncertainty due to the line shape.

\subsection{2 dc Stark Shift}

The $1 S-2 S$ transition frequency has a quadratic sensitivity to the dc electric field with the coefficient $\kappa_{\text {Stark }}=3.6 \mathrm{kHz} \mathrm{V}^{-2} \mathrm{~cm}^{2}$ (without the hyperfine structure taken into account). Although the dc Stark shift coefficients for measured hyperfine transitions in $\mathrm{H}$ and D differ only by a few percent, variations of stray fields in time may cause different shifts for the isotopes. We characterize stray fields using an additional external electric field $E_{\text {aux }}$ applied to the excitation region.

The method is illustrated in Figure 4.1(a). To apply an electric field $E_{\text {aux }}$ we use a 
plane capacitor with graphite-coated plates, one of which is grounded, and the other one is connected to a precision voltage source, whose polarity can be alternated. In the presence of a collinear stray field $E_{\text {stray }}$ the center of the parabolic dependency $f_{1 S-2 S}\left(E_{\text {aux }}\right)$ is shifted with respect to zero. This will result in the difference $\Delta f_{\text {Stark }}$ between the de Stark shifts $f_{\text {Stark }}$ measured at different polarities as

$$
\begin{aligned}
\Delta f_{\text {Stark }} & =\kappa_{\text {Stark }}\left(E_{\text {aux }}+E_{\text {stray }}\right)^{2}-\kappa\left(E_{\text {aux }}-E_{\text {stray }}\right)^{2} \\
& =4 \kappa_{\text {Stark }} E_{\text {aux }} E_{\text {stray }}
\end{aligned}
$$

Thus, the stray field may be evaluated as

$$
E_{\text {stray }}=\frac{E_{\text {aux }} \Delta f_{\text {Stark }}}{4 f_{\text {Stark }}}
$$

while the corresponding frequency shift may be deduced from the following expression

$$
\Delta f_{\text {stray }}=\kappa_{\text {Stark }} E_{\text {stray }}^{2}=\frac{\Delta f_{\text {Stark }}^{2}}{16 f_{\text {Stark }}} .
$$

The measurement of the stray fields along the $x$ axis is illustrated in Figure 4.1(b). From this measurement, we set the upper limit for the stray field along the axis to be $\left|E_{\text {stray }}\right|<6 \mathrm{mV} / \mathrm{cm}$. Repeating the measurement for all three axes we set the limit

$$
\Delta f_{\text {stray }}<1 \mathrm{~Hz}
$$

for the excitation volume restricted by the two diaphragms. It is important to mention, that using the capacitor without graphite coating plates increases the level of stray fields to $30-100 \mathrm{mV} / \mathrm{cm}$.

MC simulations show, that the quenching field in the detector region causes only a small dc Stark shift of $<0.1 \mathrm{~Hz}$ for undelayed lines. For the delayed lines the effect of the quenching field vanishes since atoms contributing to higher delays are excited in the region closer to the nozzle. Since the quench beam (Section 3.1.1) was not yet installed for this measurement, the most problematic region resides between the nozzle and the first diaphragm, where the non-coated nozzle may be a source of electric fields itself. We cannot probe this region and conservatively assume the strength of the stray electric field there to be $100 \mathrm{mV} / \mathrm{cm}$. For delay $1410 \mu$ s this causes a line shift of $5 \mathrm{~Hz}$ which we take as an uncertainty contributed by stray electric fields.

\subsection{3 ac Stark Shift}

The differential H-D ac Stark shift is negligible at our level of accuracy as long as the two isotopes are measured at the same laser power. We therefore monitor the $243 \mathrm{~nm}$ cavity transmission with a photodiode and a PMT both connected to an integrating sphere to largely reduce beam pointing effects. We fit the transition frequencies for both isotopes recorded at different laser powers by two parallel lines (compare Figure 4.2). The distances between these lines is the power independent isotope shift. 


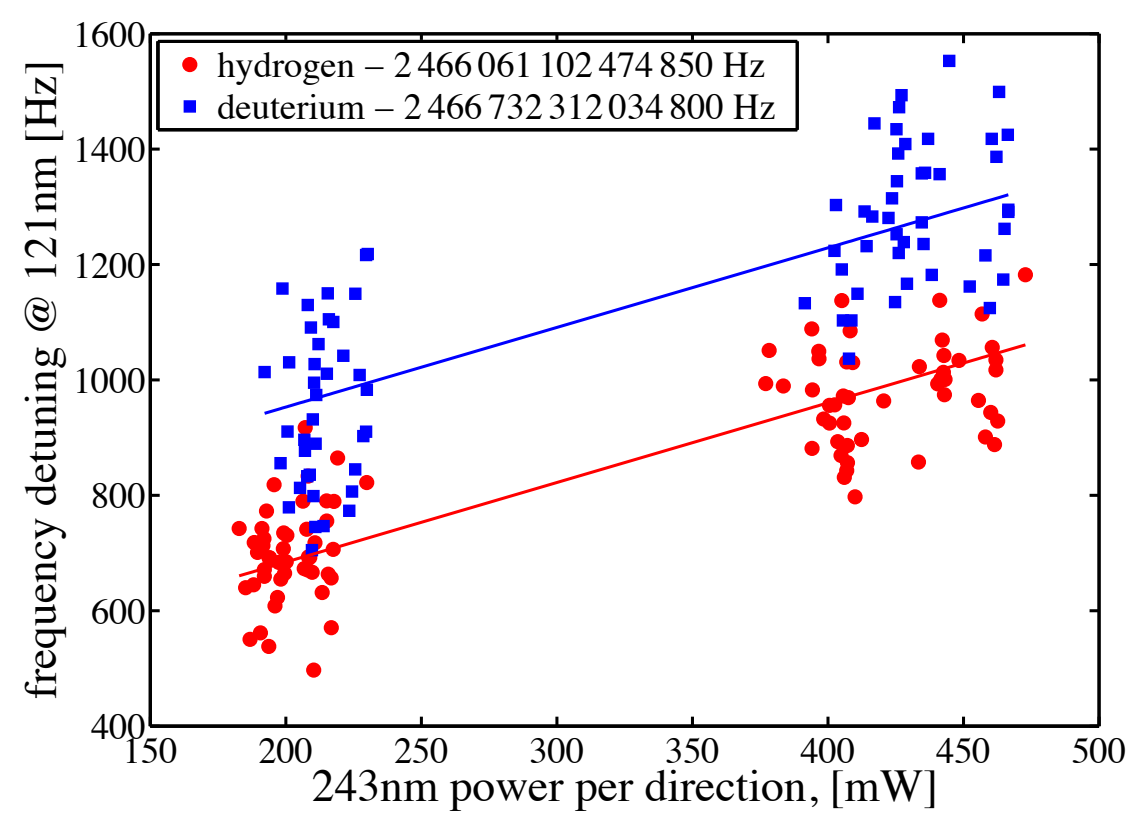

Figure 4.2: Interpolation of the ac Stark shift for one day of data. Each data point represents the center frequency of one hydrogen (red circles) / deuterium (blue squares) spectrum. The two linear fits are forced to have the same slope, the distance between them is the ac Stark independent isotope shift.

\subsubsection{Pressure Shift}

Intra-beam atomic collisions may lead to a pressure shift of the hydrogen and deuterium lines, and thermalization effects may also depend on the particle density. The data shown in Figure 4.4 (left) are recorded at the lowest possible discharge pressure of $p \approx 1$ mbar which corresponds to a flow of $1.5 \times 10^{18} \mathrm{~s}^{-1}$ for $\mathrm{H}_{2} / \mathrm{D}_{2}$ (termed regular flow). Under these conditions about $10^{17}$ particles per second leave the nozzle into the interaction region (the rest freezes on the nozzle). This corresponds to a pressure in the nozzle of $10^{-4} \mathrm{mbar}$. Using the MC simulation and the pressure shift coefficient of $-8(2) \mathrm{MHz} / \mathrm{mbar}$ [59, 60], we expect a pressure shift for each of the isotopes of $-3 \mathrm{~Hz}$ for delay $1410 \mu \mathrm{s}$. Averaging the data recorded at regular flow, we obtain $f_{1 S-2 S}^{D}-f_{1 S-2 S}^{H}=671209560203.1(5.1) \mathrm{Hz}$ where $\sigma_{0}=5.1 \mathrm{~Hz}$ is the statistical uncertainty. We also measure the isotope shift at $\mathrm{H}_{2} / \mathrm{D}_{2}$ flows increased by a factor of $\sim 3$ while maintaining the other isotope flow at regular level (see Figure 4.4 (right)).

In order to investigate density effects experimentally we perform a two-parameter linear extrapolation to zero flow (compare Figure 4.3 ) which gives $f\left(p_{\mathrm{H}}, p_{\mathrm{D}}\right)=f_{1 S-2 S}^{D}-f_{1 S-2 S}^{H}-$ $\left[11(11)+9.7(5.1) p_{\mathrm{H}}-1.7(4.9) p_{\mathrm{D}}\right] \mathrm{Hz}$ with partial pressures measured at the discharge in mbar. The $11 \mathrm{~Hz}$ uncertainty obtained by regression analysis is of the same order as $2 \sigma_{0}$. Thus, the difference $f(0,0)-\left(f_{1 S-2 S}^{D}-f_{1 S-2 S}^{H}\right)=-11(11) \mathrm{Hz}$ does not significantly deviate from zero. We therefore keep the mean value of $f_{1 S-2 S}^{D}-f_{1 S-2 S}^{H}$ as our final experimental 


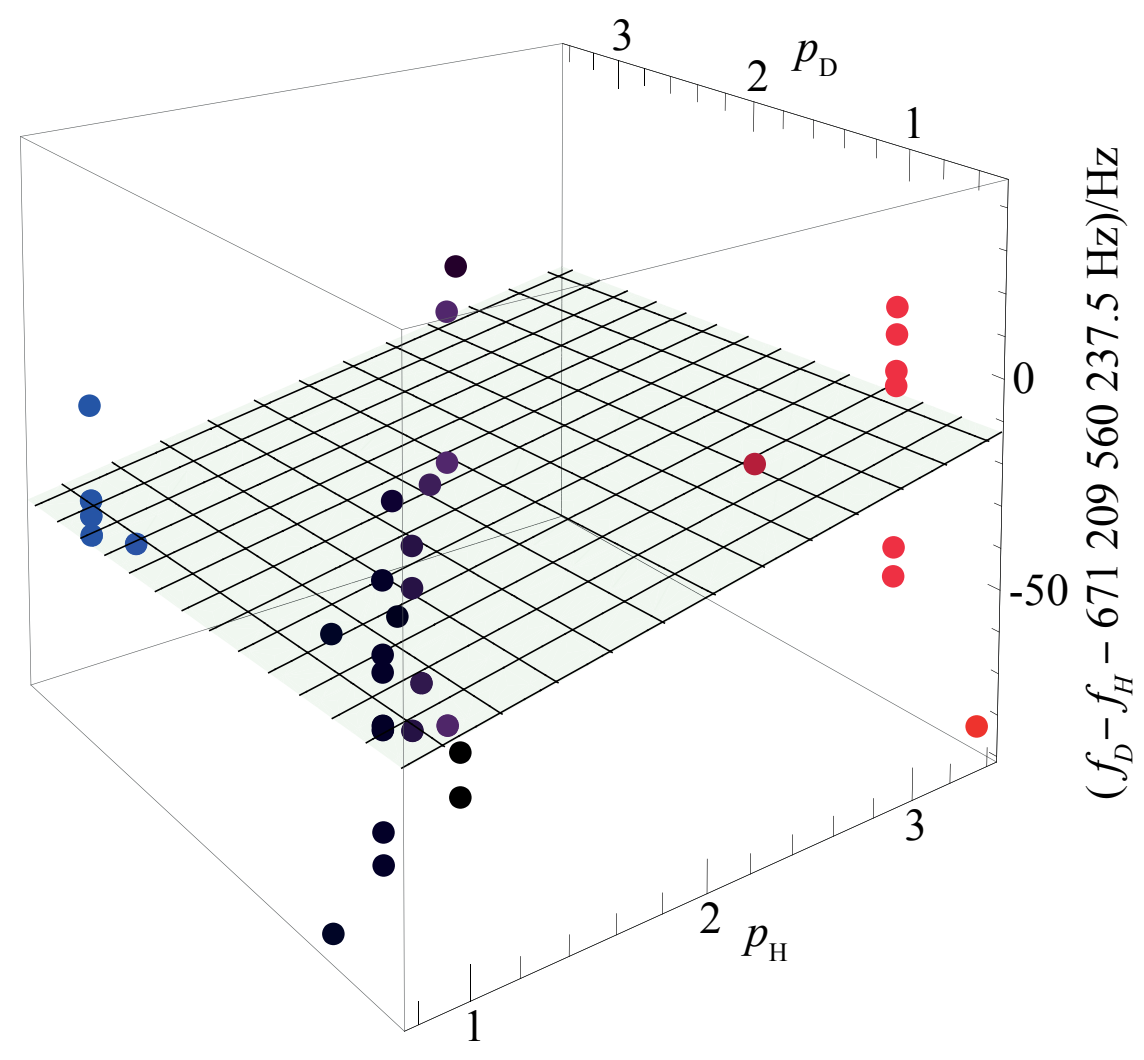

Figure 4.3: The isotope shift plotted versus hydrogen and deuterium pressure along with a fitted plain. $f\left(p_{\mathrm{H}}, p_{\mathrm{D}}\right)=f_{1 S-2 S}^{D}-f_{1 S-2 S}^{H}-\left[11(11)+9.7(5.1) p_{\mathrm{H}}-\right.$ $\left.1.7(4.9) p_{\mathrm{D}}\right] \mathrm{Hz}$. Although the error bars are not shown the plain is the result of a weighted fit.

result and add an uncertainty of $11 \mathrm{~Hz}$ due to density effects.

It should be noted, that during the isotope shift there was only one isotope present in the beam apparatus at a time. Thus, no H-D molecules should be present which could cause a substantial shift due to their large polarizability.

\subsection{Result}

The uncertainties are summarized as follows (also see Table 4.1): Statistics contributes $5.1 \mathrm{~Hz}$, from the hyperfine correction we add $2.9 \mathrm{~Hz}$, from the ac Stark shift at the reference intensity we have $1 \mathrm{~Hz}$, the second order Doppler effect contributes $6 \mathrm{~Hz}$, and the dc Stark effect gives an additional uncertainty of $5 \mathrm{~Hz}$. Together with the $11 \mathrm{~Hz}$ from the density effects, this gives

$$
\Delta f_{\exp }=670994334606(15) \mathrm{Hz}
$$


Table 4.1: Results of the $1 S-2 S$ hydrogen-deuterium frequency measurements $\left(f_{\exp }^{1997}, f_{\exp }^{2010}\right)$ and uncertainty budgets $\left(\sigma_{\exp }^{1997}, \sigma_{\exp }^{2010}\right)$ for the 1997 (Ref. [1]) and 2010 (Ref. [61]) measurements, respectively. Contributions neglected in the 1997 measurement are denoted by dashes. The frequency $\Delta f_{\mathrm{DH}}$ is the $\mathrm{H} / \mathrm{D}$ isotope shift measured for the $F=1, m_{F}= \pm 1 \rightarrow F^{\prime}=1, m_{F^{\prime}}= \pm 1$ hyperfine subcomponent.

\begin{tabular}{lcccc}
\hline \hline Contribution & $\begin{array}{c}f_{\exp }^{1997} \\
{[\mathrm{~Hz}]}\end{array}$ & $\begin{array}{c}\sigma_{\exp }^{1997} \\
{[\mathrm{~Hz}]}\end{array}$ & $\begin{array}{c}f_{\exp }^{2010} \\
{[\mathrm{~Hz}]}\end{array}$ & $\begin{array}{c}\sigma_{\exp }^{2010} \\
{[\mathrm{~Hz}]}\end{array}$ \\
\hline$\Delta f_{\mathrm{DH}}-671209560 \mathrm{kHz}$ & 225 & $\simeq 150$ & 203.1 & 5.1 \\
$\Delta f_{\mathrm{HFS}}-215225000 \mathrm{~Hz}$ & 585 & 14 & 596.5 & 2.9 \\
ac Stark shift & - & - & 0 & 1 \\
dc Stark shift & - & - & 0 & 5 \\
second order Doppler & 0 & 20 & 0 & 6 \\
density effects & - & - & 0 & 11 \\
\hline$\Delta f_{\exp }-670994334000 \mathrm{~Hz}$ & 640 & 150 & 606 & 15 \\
\hline \hline
\end{tabular}

as our final experimental result, confirming and improving the previous measurement [1] by a factor of ten. As evident from Figure 4.4, the day to day scatter of the data decreases for the second run. This is mainly due to improved statistics because the replacement of the HP/Agilent counters with continuous counters allow a larger data rate.

\subsection{Proton - Deuteron Charge Radius Difference}

According to Refs. $[22,1,52]$, the theoretical contributions to the $\mathrm{H}-\mathrm{D}$ isotope shift can be classified as (i) differences in the Dirac energy and Barker-Glover corrections [62], (ii) differences in the Lamb shifts, (iii) higher-order nuclear-size and nuclear polarizability corrections, and, finally, (iv) the main nuclear size effect given by

$$
\Delta E_{\mathrm{iv}}=\left(\frac{1}{1+m / M}\right)^{3} \frac{2 \alpha^{4} c^{4} m^{3}\left\langle r^{2}\right\rangle}{3 n^{3} \hbar^{2}},
$$

where $\alpha$ is the fine structure constant, $m$ is the mass of the orbiting lepton, and $M$ and $\left\langle r^{2}\right\rangle$ are the nuclear mass and the nuclear mean square charge radius, respectively. Following atomic physics conventions and in accordance with the definition of the nuclear radii used in our previous analysis $[51,1]$, we exclude the Barker-Glover corrections as well as the Darwin-Foldy term (which contributes $11.37 \mathrm{kHz}$ to the isotope shift [63]) from the nuclear size effects. From a nuclear physics point of view (see the paragraph below Eq. (10d) on p. 4582 of Ref. [64]), this convention corresponds to the mean square charge radius difference $\left\langle r^{2}\right\rangle_{d}-\left\langle r^{2}\right\rangle_{p}=\left\langle r^{2}\right\rangle_{\mathrm{ch}}-\left\langle r^{2}\right\rangle_{E}^{p}$, where $\left\langle r^{2}\right\rangle_{\mathrm{ch}}$ is the mean square radius of the charge distribution of the deuteron and $\left\langle r^{2}\right\rangle_{E}^{p}$ is the charge radius of the proton defined 


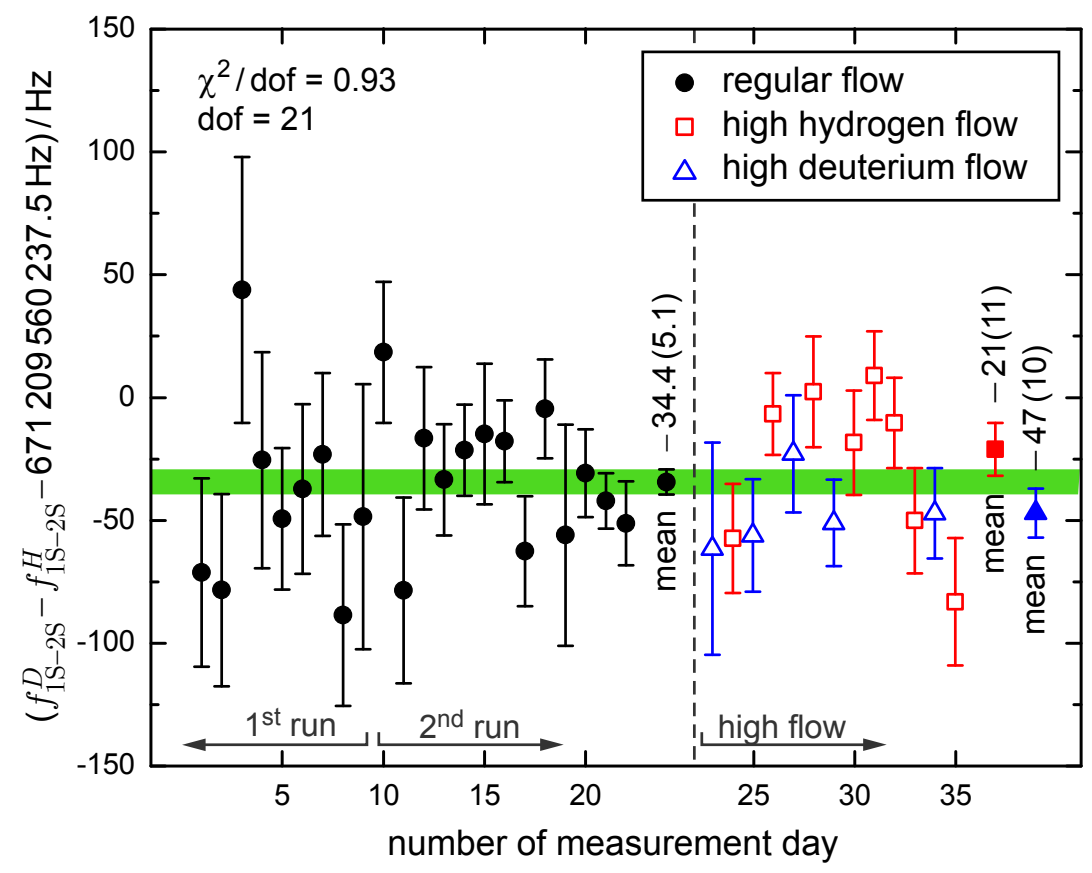

Figure 4.4: $f_{1 S-2 S}^{D}-f_{1 S-2 S}^{H}$ vs. measurement day for $\tau=1410 \mu \mathrm{s}$ not corrected for $\Delta f_{\mathrm{HFS}}$. The error bars show the statistical uncertainty, the shaded band is the $1 \sigma$ uncertainty of the mean for the regular flow data (dof - degrees of freedom). The full vertical axis corresponds to the $1 \sigma$ uncertainty of the previous measurement [1]. The data taken at higher discharge pressures are shown on the right.

via the slope of the Sachs form factor, i.e., $\left\langle r^{2}\right\rangle_{E}^{p}=6 \partial G_{E}\left(q^{2}\right) /\left.\partial q^{2}\right|_{q^{2}=0}$. The conversion of these radii to nuclear physics conventions is discussed in Ref. [64].

The theoretical isotope shift excluding $\Delta E_{\mathrm{iv}}$, i.e., the shift from groups (i) $+($ ii $)+($ iii $)$, is obtained as $[2]$

$$
\Delta f_{\mathrm{th}}=670999566.90(66)(60) \mathrm{kHz} .
$$

The current experimental values for the proton to electron and deuteron to electron mass ratios $M_{p} / m_{e}$ and $M_{d} / m_{e}$ as given in Ref. [8] yield the first $0.66 \mathrm{kHz}$ uncertainty in Eq. (4.8), due to their influence on the Dirac energy. The second theoretical uncertainty is due to sets (ii) $+\left(\right.$ iii). Higher-order recoil terms of order $(Z \alpha)^{7}(m / M) \log (Z \alpha)^{-2}$ and higher-order radiative-recoil corrections contribute $0.34 \mathrm{kHz}$. In our evaluation, we use the result given in Refs. $[65,66,67]$ for the radiative recoil of order $\alpha(Z \alpha)^{5} m_{e} c^{2}$. The deuteron polarizability is determined according to [54]. Here, we take into account a larger uncertainty of $0.5 \mathrm{kHz}$ in order to account for the possibility of nuclear polarizability effects in diagrams with inelastic multi-photon exchanges according to Refs. [1, 52, 53, 68]. The logarithmic divergence of the form factor is subtracted according to Eq. (69) of Ref. [52].

The difference of the experimental and theoretical value $\Delta f_{\mathrm{th}}-\Delta f_{\exp }=5232.29(89) \mathrm{kHz}$ 
is associated exclusively with $\Delta E_{\mathrm{iv}}$, and the result

$$
\left\langle r^{2}\right\rangle_{d}-\left\langle r^{2}\right\rangle_{p}=3.82007(65) \mathrm{fm}^{2}
$$

confirms the result of Ref. [1] and constitutes a more than twofold improvement in accuracy.

Our value for the deuteron structure radius defined according to Eq. (11) of Ref. [1] is

$$
\begin{aligned}
\left\langle r^{2}\right\rangle_{\mathrm{str}} & =\left\langle r^{2}\right\rangle_{d}-\left\langle r^{2}\right\rangle_{n}-\left\langle r^{2}\right\rangle_{p}-\frac{3 \hbar^{2}}{4 m_{p}^{2} c^{2}}, \\
r_{\mathrm{str}} & =1.97507(78) \mathrm{fm}
\end{aligned}
$$

where $\left\langle r^{2}\right\rangle_{n}=-0.114(3) \mathrm{fm}^{2}$ is the neutron charge radius [69, 70]. The last term on the right-hand side of Eq. (4.10a) corresponds to the Darwin-Foldy correction for the proton.

We find that the uncertainty is dominated by the experimental uncertainties of the $M_{p} / m_{e}$ and $M_{d} / m_{e}$ mass ratios as well as the theoretical uncertainty in the nuclear structure effects while the measurement of the isotope shift exceeds the accuracy currently needed. From theory, more accurate calculations of nuclear structure effects including multi-photon exchange diagrams and improved measurements of mass ratios are clearly needed. 


\section{Chapter 5}

\section{Absolute Frequency Measurement}

\subsection{Introduction}

For the last six decades, spectroscopy on atomic hydrogen along with its calculable atomic structure has been fueling the development and testing of quantum electro-dynamics (QED) and has lead to a precise determination of the Rydberg constant and the proton charge radius [71]. The absolute frequency of the $1 S-2 S$ transition has been measured with particularly high precision, so that it now serves as a corner stone in the least squares adjustment of the fundamental constants [8]. The resonance has been used to set limits on a possible variation of fundamental constants [3] and violation of Lorentz boost invariance [72]. It further promises a stringent test of the charge conjugation/parity/time reversal (CPT) theorem by comparison with the same transition in antihydrogen $[73,16]$.

Here, we present a more than three times more accurate measurement of the $1 S-2 S$ transition as compared to the previous best measurements [13, 3], now reaching $4.2 \times 10^{-15}$. The key improvements are (a) the replacement of a dye laser with a diode laser system with improved frequency stability for the two-photon spectroscopy, (b) a direct measurement of the $2 S$ velocity distribution of the thermal atomic hydrogen beam which allows a more accurate characterization of the second order Doppler effect (SOD), and, learning from the isotope shift measurement, (c) the introduction of a quench laser, resetting the population to the ground state just before the entrance of the excitation region, removing possible frequency shifts due to the high density of atoms and a possible dc Stark shift from patch charges within the nozzle (Section 4.3.2).

\subsection{Experimental Summary}

\subsubsection{Beam Apparatus}

The beam apparatus is described in Section 3.1. The quenchbeam (Section 3.1.1) near $486 \mathrm{~nm}$ is used to reset atoms via the $4 P$ state to the ground state right at the entrance to the excitation region. Lyman- $\alpha$ photons are detected with the photo-multiplier tube 
as shown in Figure 3.1. After the measurement campaign it was discovered that the polarization of the spectroscopy light is slightly mis-aligned with respect to the vacuum entrance window's Brewster angle so that $10 \%$ of laser power was lost at this window.

\subsubsection{Laser System and Frequency Measurement}

The $1 S-2 S$ transition is excited with the forth harmonic of ECDL1 (Section 3.2). Light from ECDL1 is sent to the frequency comb through a $10 \mathrm{~m}$ long, unstabilized single-mode fiber. The influence of fiber fluctuations was measured to be on the $10^{-18}$ level. The frequency of ECDL1 with respect to the hydrogen maser can be calculated by

$$
f_{a b s}^{H}=8 \times\left(N_{\mathrm{H}} f_{\mathrm{rep}}+2 f_{\mathrm{CEO}}+f_{\mathrm{FP} 1}+f_{\mathrm{LO}}^{H}+2 f_{\mathrm{AOM} 1}\right) .
$$

with the frequency comb mode number $N_{\mathrm{H}}=1233027$, the repetition rate $f_{\text {rep }}=$ $250 \mathrm{MHz}$, and its offset frequency $f_{\mathrm{CEO}}=30 \mathrm{MHz}$. The laser frequency is shifted with respect to the cavity by AOM 1 (Figure 3.4 ) with a frequency of $F_{\mathrm{AOM} 1} \approx 360 \mathrm{MHz}$. The beat between the frequency comb line and ECDL1 is $f_{\mathrm{FP} 1} \approx 29 \mathrm{MHz}$ after mixing it down with a local oscillator $f_{\mathrm{LO}}^{H}=78.674368 \mathrm{MHz}$. This odd number is historically used and serves several fiber noise cancellation schemes throughout the lab.

\subsubsection{Measurement Procedure}

\section{Preparation}

To ensure the hydrogen spectroscopy machine was performing as expected when the mobile cesium fountain clock FOM arrived at MPQ, we started full data taking operation one week before the scheduled FOM arrival. This time proved to be well invested since we then were very confident in operating the machine by the time FOM was operational.

\section{Data Taking}

$15871 S-2 S$ spectra have been recorded during twelve consecutive days starting on May 30 2010 with a break on June 9. On each measurement day, the vacuum chamber was opened early in the morning to clean the mirrors of the enhancement cavity. Also, before the first and seventh day of measurement, the copper nozzle was polished on the inside and the Teflon tubing was cleaned in soap water.

By 10 a.m., the cryo-pump was switched on and the experiments started at around 4 p.m.. During the measurement, the transmission of the enhancement cavity would drop and after 70 to 100 line pairs, the experiment was stopped. During the night, the cryopump was reheated to evaporate the accumulated hydrogen.

The data of June 9 was excluded from the analysis since the frequency comb was running in an unfavorable mode-lock regime where the beat note between ECDL1 and the comb had an extra side-peak within the filter bandwidth of the counter. Since the two peaks were separated by less than $1 \mathrm{MHz}$ they are seen similarly with the two filters of 


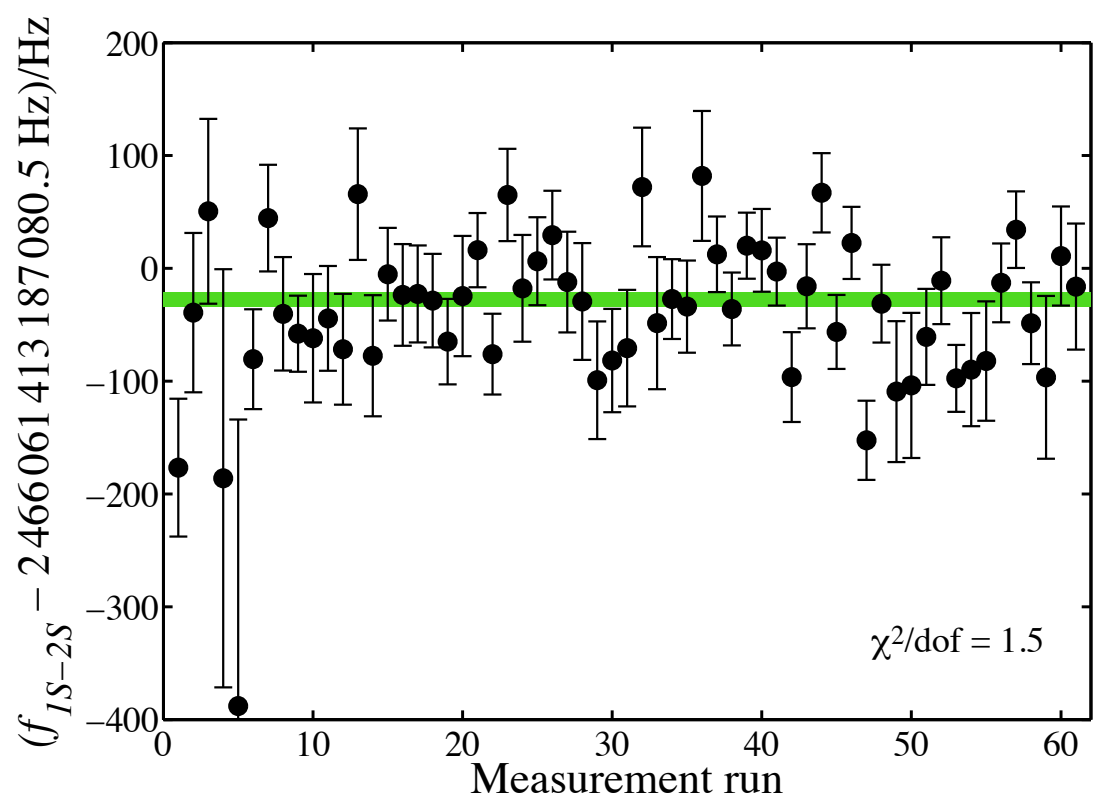

Figure 5.1: Absolute frequency data. Shown is the statistical uncertainty only. Each data point represents the weighted average for delays $\tau_{5}=810 \ldots 1010 \mu \mathrm{s}$ and $\tau_{6}=1010 \ldots 1210 \mu \mathrm{s}$. The green shaded band represents the $1 \sigma$ uncertainty around the weighted mean. The reduced $\chi^{2} /$ dof $=1.5$

different bandwidth used for cycle slip detection [56]. Therefore, the problem is invisible to the cycle slip detection and a reliable counting cannot be guaranteed.

\subsection{Characterization of Uncertainties}

This Section describes the data evaluation as well as the characterization of systematic effects for the first $1 S-2 S$ absolute frequency measurement. It is important to note, that in both absolute frequency measurements, delay has changed its meaning in comparison to previous measurements. Now, delay means a certain arrival time interval which starts at a certain delay and ends $200 \mu$ s later*. Consequently, the $2 S$ counts are sorted into twelve time bins $\tau_{1}=10 \ldots 210 \mu \mathrm{s}, \tau_{2}=210 \ldots 410 \mu \mathrm{s}, \ldots, \tau_{12}=2210 \ldots 2410 \mu \mathrm{s}$. For the final result, we will use delays $\tau_{5}=810 \ldots 1010 \mu$ s and $\tau_{6}=1010 \ldots 1210 \mu$ s only. This is motivated by the correction of second order Doppler effect and ac Stark shift as will be explained in Section 5.3.3.

The two dominating systematic uncertainties in our experiment are the ac Stark shift and the second order Doppler effect. Here, we discuss the compensation of these two main systematic effects and estimations of the remainder. The Doppler effect, due to the velocity

${ }^{*}$ Previously, delay $\tau_{i}=((i-1) \cdot 200+10) \mu$ s to $\infty$. Consequently, the delays were not independent, since delay $\tau_{i}$ included all data contained in $\tau_{i+1}$. 

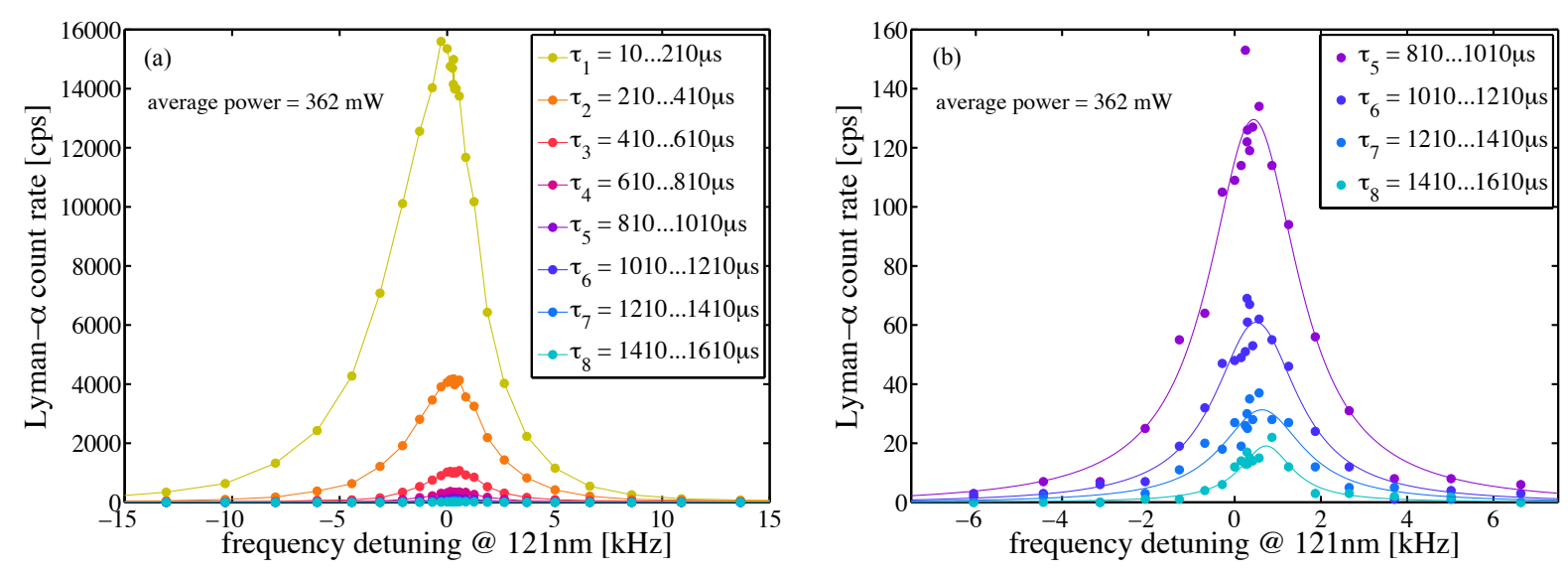

Figure 5.2: $1 S-2 S$ line profiles for various delays. (a) One can clearly see the asymmetry for short delays due to the quadratic nature of the second order Doppler effect in conjunction with the Maxwellian velocity distribution. The line is a guide to the eye. (b) For high delays the line become more symmetric and a Lorentzian fit (solid line) represents an adequate approximation of the line shape.

$v$ of the atoms, is cancelled to first order by virtue of the two-photon excitation scheme [74]. The remaining second order Doppler shift $\Delta f_{\mathrm{dp}}=-v^{2} f_{1 S-2 S} /\left(2 c^{2}\right)$ is compensated in two steps: First, we chop the excitation light at $160 \mathrm{~Hz}$ (see Fig. 3.1) which allows time-offlight resolved detection of atoms excited to the $2 S$ state. Evaluating only $2 S$ counts recorded at a certain delay $\tau$ after the light has been switched off by the chopper wheel, allows the fastest atoms to escape. This samples the slow tail of the velocity distribution and removes most of the SOD. Second, to further study and subtract the SOD, we have independently measured the velocity distribution of the $2 S$ atoms as described below. We evaluate the resonance data in each of the twelve time-of-flight ranges $\tau_{1}=10 \ldots 210 \mu \mathrm{s}$, $\tau_{2}=210 \ldots 410 \mu \mathrm{s}, \ldots, \tau_{12}=2210 \ldots 2410 \mu$ s independently, which provides a test of the SOD correction. The residual uncertainty of this procedure was determined by evaluating a second data set that was generated using a Monte Carlo (MC) simulation [58, 75] in exactly the same way. For various simulation parameters such as temperature, geometry and initial $1 S$ velocity distributions we find the uncertainty to be smaller than $2.0 \times 10^{-15}$ (Appendix C) which is below the current statistical uncertainty of $2.6 \times 10^{-15}$ (Table 5.1).

\subsubsection{Quadratic ac Stark Shift due to the Spectroscopy Laser}

The ac Stark shift is mostly linear in laser power $P$. However, a small quadratic contribution [58] must be taken into account, before we can apply a linear extrapolation using the stable, but otherwise not precisely calibrated laser power readings. The main contribution to the quadratic ac Stark shift is due to ionization of the $2 S$ atoms by a third $243 \mathrm{~nm}$ 


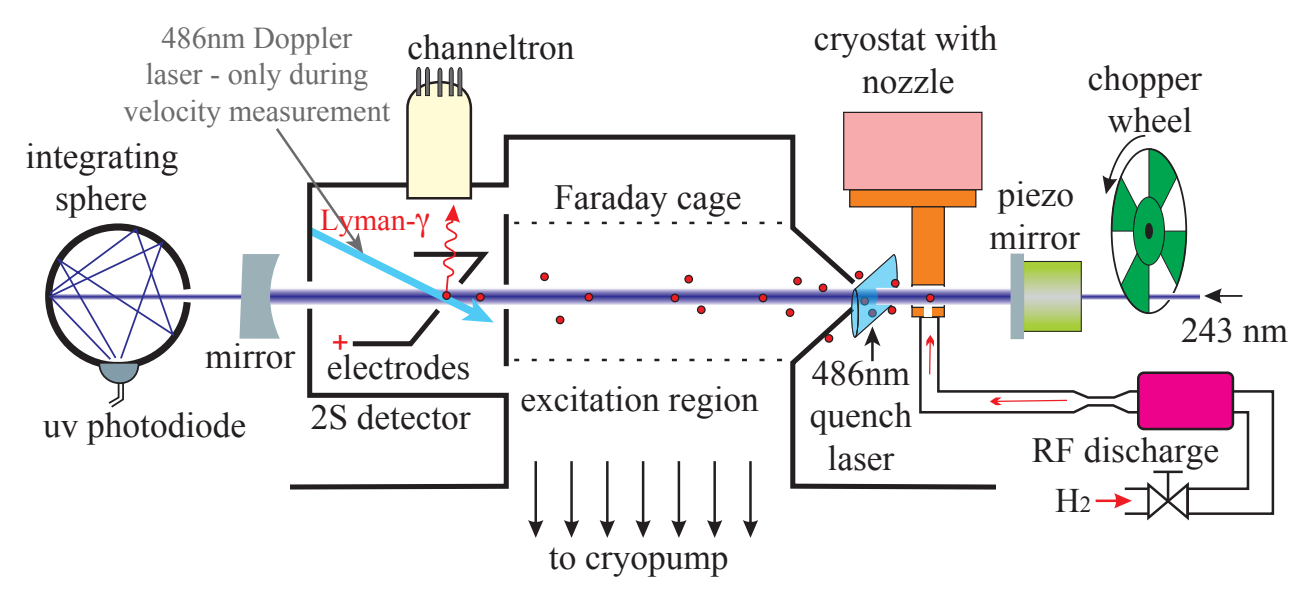

Figure 5.3: Setup for measuring the $2 S$ velocity distribution. A Doppler laser near $486 \mathrm{~nm}$ is crossed with the atomic beam at an angle of $45^{\circ}$ right between the quench electrodes which are grounded for the measurement. The $4 P$ state decays with $90 \%$ branching ratio to the $1 S$ state. The emitted Lyman- $\gamma$ photons are detected with a channeltron.

photon that removes preferably atoms that see larger laser powers. For excitation laser powers of $300 \mathrm{~mW}$ as present in our experiment, the quadratic ac Stark shift contributes on the order of $1 \times 10^{-14}$ as derived from the MC simulations. This is sufficiently small to rely on these simulations that assume a Maxwell distribution for the $1 S$ atoms using the absolute laser power within $20 \%$ relative uncertainty. Modeling and subtracting the delay dependent quadratic ac Stark effect in this way then allows to linearly extrapolate the line centers, without knowing the exact laser power calibration. This procedure reduces the overall ac Stark shift uncertainty to $0.8 \times 10^{-15}$, again estimated by evaluating artificial data (see Appendix C).

\subsubsection{Line Shape}

Due to the lack of a complete line shape model (compare Appendix C) we find the experimental (and simulated) line centers by fitting Lorentzians which represent a good approximation of the line shape for delays $\tau_{4}=610 \ldots 810 \mu$ s and higher (compare Figure 5.2). For lower delays the second order Doppler effect causes an asymmetry so we do not evaluate them. A small residual asymmetry for the longer delays is determined and taken into account by comparing with the MC simulation. Again, we use this simulation only for small corrections. In Appendix $\mathrm{C}$ a careful investigation shows that the errors introduced by fitting a Lorentzian can be compensated. 

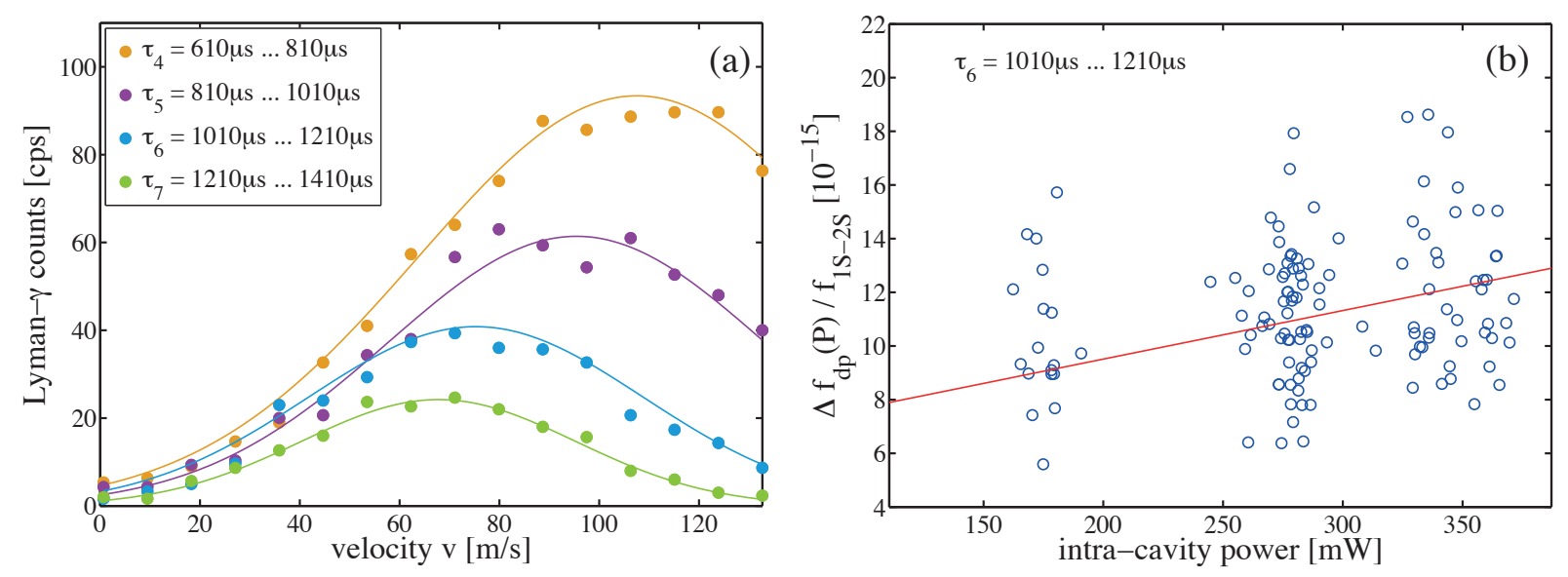

Figure 5.4: (a) Low velocity part of the Doppler profiles of $2 S$ atoms recorded for different delays $\tau$ at $370 \mathrm{~mW}$ intra-cavity power along with a Gaussian fit. Atoms with higher velocities fall into shorter delays which we do not use in the final evaluation. (b) Fractional second order Doppler correction $\Delta f_{\mathrm{dp}}(P) / f_{1 S-2 S}$ versus intra-cavity power for delay $\tau_{6}=1010 \mu \mathrm{s} \ldots 1210 \mu \mathrm{s}$. The power dependence arises from ionization losses of slow atoms. Each point represents the Doppler correction as calculated from the central velocity of a single velocity profile measurement along with a linear fit.

\subsubsection{Second Order Doppler Effect}

\section{Measurement of the $2 \mathrm{~S}$ Velocity Distribution}

To correct the second order Doppler effect $\Delta f_{\mathrm{dp}}=-v^{2} f_{1 S-2 S} /\left(2 c^{2}\right)$, an accurate understanding of the velocity distribution is desirable. Previously, this information has been extracted from the line shape of the $2 S$ spectra with an uncertainty of $8 \times 10^{-15}$ [13]. Here, we measure the $2 S$ velocity distribution directly via the first order Doppler effect on the $2 S-4 P$ one photon transition which we excite at an angle of $45^{\circ}$ near $486 \mathrm{~nm}$ (Doppler laser in Figure 5.3). The $2 S-4 P$ transition has a sufficiently narrow natural line width of $13 \mathrm{MHz}$ (corresponding to $\Delta v=8 \mathrm{~m} / \mathrm{s}$ at $45^{\circ}$ ) to resolve velocities on the level of $1 \mathrm{~m} / \mathrm{s}$.

The $4 P$ state decays to the ground state with a $90 \%$ branching ratio emitting a $97 \mathrm{~nm}$ Lyman- $\gamma$ photon which can be easily detected using a channeltron. Pulsing the $486 \mathrm{~nm}$ Doppler laser with an AOM (rise and fall time are measured to be $250 \mathrm{~ns}$ ) avoids power broadening (and loss of velocity resolution) while providing equal quench probability for atoms of different velocity. We use $15 \mu$ s long pulses at a rate of $5464 \mathrm{~Hz}(183 \mu \mathrm{s})$. This pulse repetition rate ensures that the Moire pattern resulting from the pulsed AOM (486 nm) and the $160 \mathrm{~Hz}$ chopper $(243 \mathrm{~nm})$ averages out within the recording time of 170 chopper cycles. With a $486 \mathrm{~nm}$ laser beam diameter $\left(1 / e^{2}\right)$ of $w=2 \mathrm{~mm}$ this ensure equal excitation rates for atoms between $12 \mathrm{~m} / \mathrm{s}$ and $130 \mathrm{~m} / \mathrm{s}$. The power in the Doppler laser is $12 \mu \mathrm{W}$.

We cross the $486 \mathrm{~nm}$ beam with the atomic beam right between the quench electrodes 

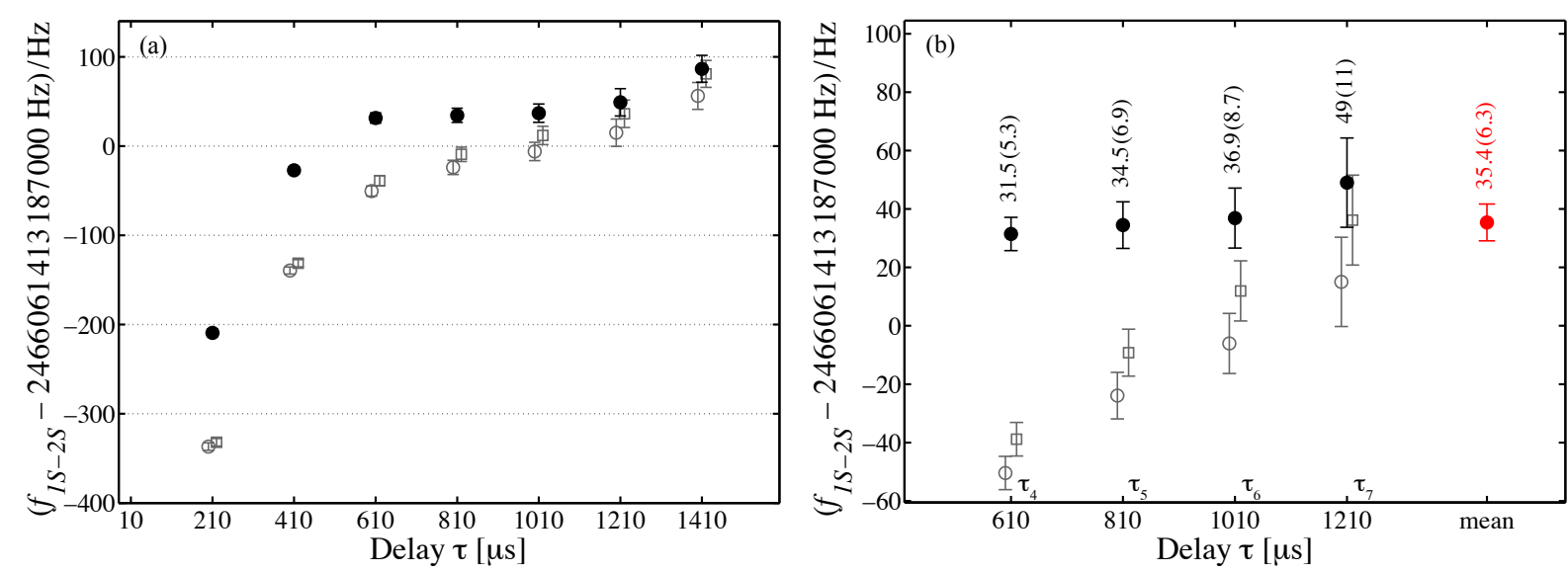

Figure 5.5: The plot shows $f_{1 S-2 S}$ with all corrections applied. The error bars represent the $1 \sigma$ statistical uncertainty. The open circles show the same data with the second order Doppler effect not corrected, the open squares do neither include the second oder Doppler effect nor the quadratic ac Stark shift corrections. (a) The correction only works for delays $\tau_{4}$ to $\tau_{7}$ (see text). (b) Zoom in for delays $\tau_{4}=610 \ldots 810 \mu$ s to $\tau_{7}=1210 \ldots 1410 \mu$ s (filled points) along with the mean as calculated from delays $\tau_{5,6}$ (see text).

which are grounded for the velocity distribution measurements. Using the same delayed detection as for the $1 S-2 S$ spectra allows to extract the velocity distribution of $2 S$ atoms that contribute to the signal with delay $\tau$. Working with the excited state directly gives the advantage of measuring the convolution of the velocity distribution with the excitation probability making the simulation of excitation dynamics unnecessary for this purpose. The low velocity part of a typical Doppler profile for delays $\tau_{4}=610 \ldots 810 \mu$ s to $\tau_{7}=$ $1210 \ldots 1410 \mu \mathrm{s}$ is shown in Figure 5.4 (a).

\section{Determination of the Second Order Doppler Effect Correction}

From 131 recorded Doppler profiles $p_{\tau}(v, P)$ we calculate the second order Doppler effect for each delay $\tau$ according to $\Delta f_{\mathrm{dp}}(P)=-v_{\mathrm{c}}^{2}(P) f_{1 S-2 S} /\left(2 c^{2}\right)$ where the central velocity $v_{\mathrm{c}}$ is determined by a Gaussian fit to the velocity profiles (see Figure $5.4(\mathrm{a})$ ). The power dependence arises from ionization losses of slow atoms. A linear fit to $\Delta f_{\mathrm{dp}}(P)$ reveals the second order Doppler effect correction as shown in Figure 5.4(b).

\section{Uncertainty Estimation of the Second Order Doppler Effect}

The uncertainty in the second order Doppler correction is caused by three main sources. First, the statistical uncertainty obtained from linear regression analysis of $\Delta f_{\mathrm{dp}}(P)$ contributes $1.7 \times 10^{-15}$. Second, during the velocity measurements the $1 S-2 S$ spectroscopy laser was kept on the resonance only within $\pm 160 \mathrm{~Hz}$. The MC simulation reveals an asso- 
ciated uncertainty of $0.8 \times 10^{-15}$. Third, the $45^{\circ}$ angle between the atomic beam and the laser beam used to measure the velocity distribution can only be adjusted within $\pm 1^{\circ}$. This translates to an uncertainty in the second order Doppler effect of $0.8 \times 10^{-15}$. Summing in quadrature leads to an overall uncertainty of the second order Doppler correction of $2.0 \times 10^{-15}$.

\section{Test of the Second Order Doppler Effect Correction}

The fully corrected data is shown in Figure 5.5. The transition frequency $f_{1 S-2 S}$ is independent of the delay for $\tau_{4}=610 \ldots 810 \mu$ s to $\tau_{7}=1210 \ldots 1410 \mu$ s unlike before the correction. The insignificant remaining slope can be readily explained within the uncertainty in the correction of the second order Doppler effect. For the final analysis we only use delays $\tau_{5,6}$. For delays $\tau_{1 \ldots 4}$ the second order Doppler effect cannot be sufficiently characterized. For delay $\tau_{7}$ and higher, the quadratic ac Stark shift correction cannot be extracted with competitive uncertainty due to the inaccuracy of the absolute power measurement. Also, the statistics is poor for these delays.

\subsection{4 ac Stark Shift by $\mathbf{4 8 6} \mathrm{nm}$ Quench Light}

The $486 \mathrm{~nm}$ quench beam can cause an ac Stark shift on the $1 S-2 S$ transition itself. Here, two possible mechanisms come to mind. First, we experimentally characterize a possible shift due to $486 \mathrm{~nm}$ light which is scattered along the atomic beam. For this measurement, we record two lines simultaneously - in analogy to the $243 \mathrm{~nm}$ power modulation - for which we greatly change the quench beam's intensity and therefore the amount of scattered light. We do not find a significant shift on the $0.4 \times 10^{-15}$ level. Second, atoms leaving the quench beam experience a low intensity region at the far wing of the Gaussian beam profile at which they will not be quenched but only shifted by the $486 \mathrm{~nm}$ radiation. We numerically simulate this effect by extending our standard MC simulation by a spatially dependent quench rate $\gamma_{q}$ by modifying the optical Bloch equations

$$
\begin{aligned}
\frac{\partial}{\partial t} \rho_{g g}^{\prime} & =-\Omega \Im\left(\rho_{g e}^{\prime}\right)+\left(\gamma_{s}+\gamma_{q}\right) \rho_{e e}^{\prime} \\
\frac{\partial}{\partial t} \rho_{g e}^{\prime} & =i \Delta \omega \rho_{g e}^{\prime}+i \frac{\Omega}{2}\left(\rho_{g g}^{\prime}-\rho_{e e}^{\prime}\right)-\frac{\gamma_{i}+\gamma_{s}+\gamma_{q}}{2} \rho_{g e}^{\prime} \\
\frac{\partial}{\partial t} \rho_{e e}^{\prime} & =\Omega \Im\left(\rho_{g e}^{\prime}\right)-\left(\gamma_{i}+\gamma_{s}+\gamma_{q}\right) \rho_{e e}^{\prime}
\end{aligned}
$$

where $\rho$ is the density matrix of the two-level system with states $g$ and $e, \Omega$ is the twophoton Rabi frequency, $\Delta \omega$ is the laser detuning and $\gamma_{s}$ and $\gamma_{i}$ are spontaneous decay and ionization rates, respectively. Also, an ac Stark shift due to the quench laser is introduced

$$
\Delta \omega \rightarrow \Delta \omega+2 \pi \Delta \nu_{\mathrm{ac}}^{486}
$$

We find no significant shift with an uncertainty of

$$
\Delta f / f_{1 S-2 S} \leq 0.81 \times 10^{-15} \text {. }
$$




\subsubsection{Hyperfine Correction}

To calculate the hyperfine centroid from the measured $F=1, m_{F}= \pm 1$ to $F=1, m_{F}= \pm 1$ transition one has to add

$$
\Delta f_{\mathrm{HFS}}^{\mathrm{H}}=+310712229.4(1.7) \mathrm{Hz}
$$

as described in Section 2.3.

\subsection{6 dc Stark Shift}

The measurement of stray electric fields which could cause a dc Stark shift is described in Section 4.3.2. The dc Stark shift within the excitation region was measured to be $1 \mathrm{~Hz}$ $\left(0.4 \times 10^{-15}\right)$. However, for the isotope shift measurement we had to add an uncertainty of $2 \times 10^{-15}$ to take into account possible stray electric fields in the space between nozzle and front aperture [2]. Utilizing the additional quench beam installed for the $1 S-2 S$ absolute frequency meausrement (Section 3.1.1), we can drop this extra contribution here.

\subsubsection{Pressure Shift}

During the hydrogen/deuterium $1 S-2 S$ isotope shift measurement [61] a pressure shift was one of the limiting systematics (Section 4.3.4). The pressure shift for this experiment has been greatly reduced by quenching $2 S$ atoms from the high pressure region within the nozzle and the first centimeter between the nozzle orifice and the front aperture. With an atom flux of $\sim 10^{17}$ particles per second, a nozzle diameter of $2.2 \mathrm{~mm}$ and the pressure shift coefficient of $-8(2) \mathrm{MHz} / \mathrm{mbar}$ as measured in a cell [76] we find the pressure shift to be well below $0.4 \times 10^{-15}$.

\subsubsection{Zeeman Shift due to an Asymmetry in the Populations of the $\mathbf{m}_{\mathrm{F}}=+1$ and $\mathbf{m}_{\mathrm{F}}=-1$ States}

As explained in Section 2.3, applying an external magnetic field of $0.5 \mathrm{mT}$ to split the magnetic field insensitive hyperfine transitions $F=1, m_{F}= \pm 1$ to $F^{\prime}=1, m_{F^{\prime}}= \pm 1$ which we use for spectroscopy from the magnetic field sensitive transitions $\left(F=1, m_{F}=\right.$ $\left.0 \rightarrow F^{\prime}=1, m_{F^{\prime}}=0\right)$. The hyperfine centroid can be obtained by adding $\Delta f_{\mathrm{HFS}}=$ $+310712229.4(1.7) \mathrm{Hz}$ as calculated from the experimental results for the $1 S$ and $2 S$ hyperfine splittings $[27,26]$.

The hyperfine transitions $F=1, m_{F}= \pm 1 \rightarrow F^{\prime}=1, m_{F^{\prime}}= \pm 1$ experience a Zeeman shift of $\pm 360 \mathrm{~Hz} / \mathrm{mT}$ which consequently averages to zero for equal populations in both hyperfine Zeeman components. Any asymmetry in these populations would directly lead to a frequency shift of the measured transition. To test this, we have performed a dedicated experiment in which we increase the applied magnetic field by a factor of 3.125 by increasing the number of windings in the coil used to generate the bias magnetic field. Then, in a similar way to the modulation of the excitation power, we reverse the magnetic field for 


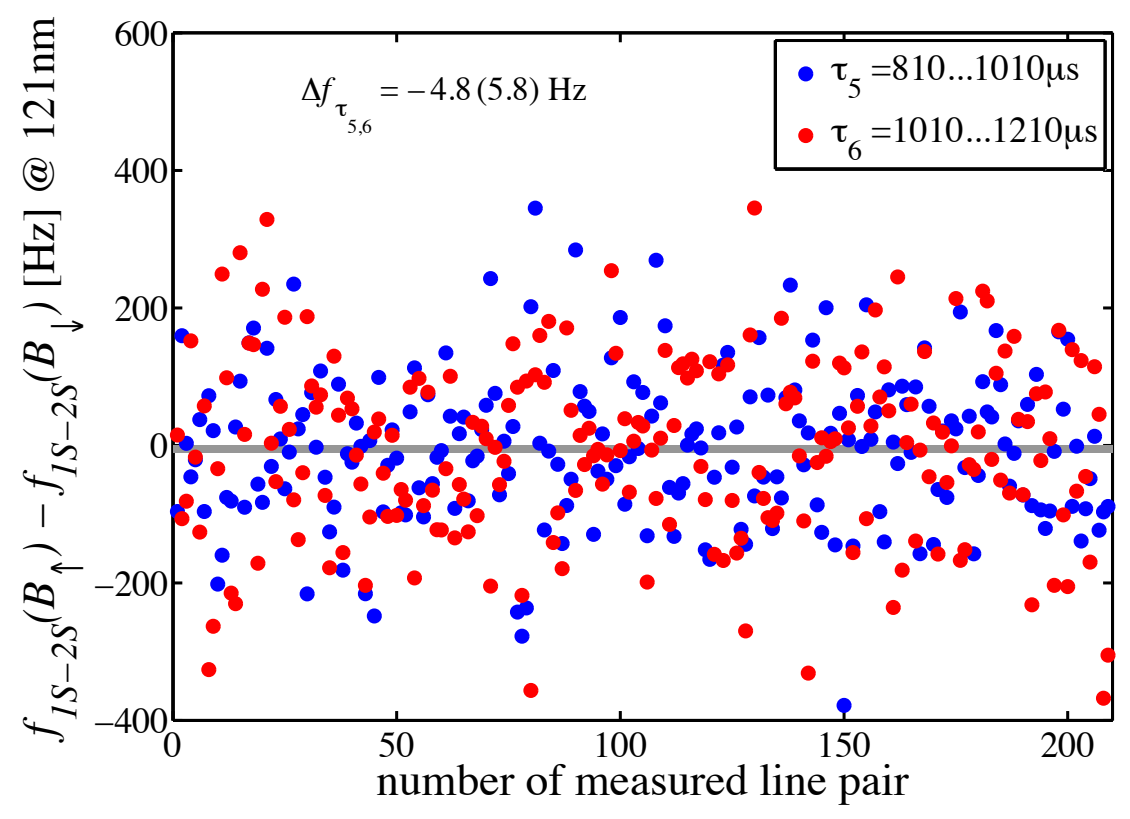

Figure 5.6: Measurement of the asymmetry in the populations of the $1 S m_{F}=+1$ and $m_{F}=-1$ States. Shown is the difference of the $1 S-2 S$ transition frequency for the magnetic field pointing upward in the laboratory $\left(f_{1 \mathrm{~S}-2 \mathrm{~S}}\left(B_{\uparrow}\right)\right)$ and downward $\left(f_{1 \mathrm{~S}-2 \mathrm{~S}}\left(B_{\downarrow}\right)\right)$ for delay $\tau_{5}=810 \ldots 1010 \mu \mathrm{s}$ and $\tau_{6}=1010 \ldots 1210 \mu \mathrm{s}$. The shaded band represents the mean over both delays with the $1 \sigma$ statistical uncertainty.

every second point while sampling the $2 S$ resonance profile. This differential measurement proves to be highly stable. Within 210 profiles we can restrict the frequency difference to $\Delta f_{\tau_{5,6}}=-4.8(5.8) \mathrm{Hz}$ (see Figure 5.6). Dividing by 6.25 for the increased magnetic field we find the Zeeman shift to be compatible with zero within its uncertainty of

$$
\Delta f_{\text {Zeeman }} / f_{1 S-2 S}=0.38 \times 10^{-15}
$$

corresponding to $\Delta f_{\text {Zeeman }}=0.93 \mathrm{~Hz}$ for the $0.5 \mathrm{mT}$ field applied during a regular measurement.

\subsubsection{Blackbody Shift}

From Farley and Wing (Ref. [77], Table I) we can directly read the frequency shift of the $1 S-2 S$ transition in atomic hyrogen due to blackbody radiation at $300 \mathrm{~K}$ to be $\Delta f_{\mathrm{BBR}}=$ $-1.03 \mathrm{~Hz}$. Assuming a temperature uncertainty of $\pm 30 \mathrm{~K}$ we find $\Delta f_{\mathrm{BBR}}=-1.0(0.3) \mathrm{Hz}$ which translates to

$$
\Delta f_{\mathrm{BBR}} / f_{1 S-2 S}=-4.1(1.2) \times 10^{-16} .
$$

We correct for the blackbody shift. 


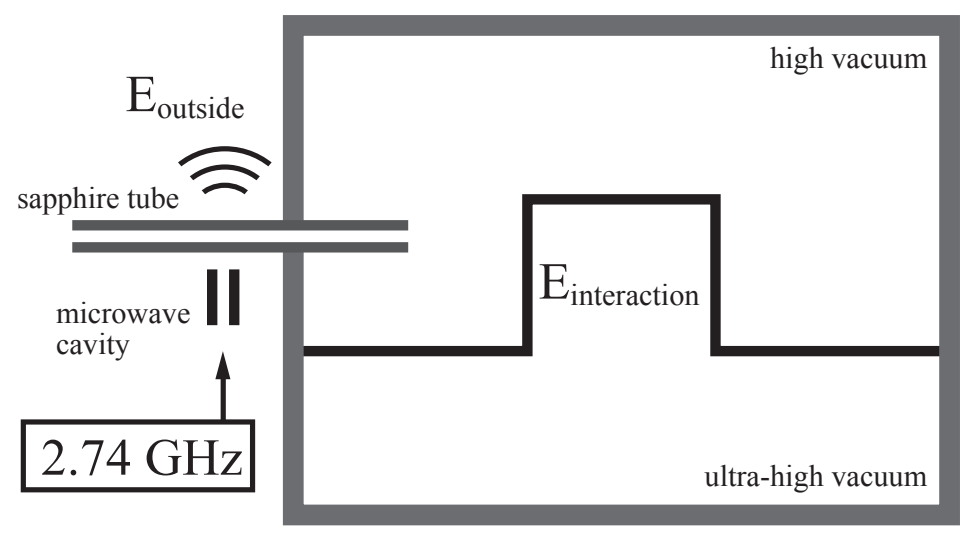

Figure 5.7: The microwave discharge is operated outside of the vacuum chamber. The vacuum chamber wall as well as the excitation region enclosure attenuate the microwave radiation by four and three orders of magnitude, respectively.

From Vanier's book [78] we find the blackbody shift on the hyperfine splitting of the $1 S$ state to be $\Delta f_{\mathrm{BBR}}^{\mathrm{HFS}, 1 S} \approx 10^{-7} \mathrm{~Hz}\left(\Delta f_{\mathrm{BBR}}^{\mathrm{HFS}, 1 S} / f_{1 S-2 S} \approx 10^{-22}\right)$ and that of the $2 S$ state $\Delta f_{\mathrm{BBR}}^{\mathrm{HFS}, 2 S} \approx 10^{-8} \mathrm{~Hz}\left(\Delta f_{\mathrm{BBR}}^{\mathrm{HFS}, 2 S} / f_{1 S-2 S} \approx 10^{-23}\right)$. Both contributions are negligible.

\subsubsection{0 ac Stark Shift due to the Radio Frequency Discharge}

For hydrogen dissociation we use a microwave source delivering $30 \mathrm{~W}$ microwave radiation at $2.4 \mathrm{GHz}$. Here, it shall be shown that the influence on the $1 S-2 S$ transition is negligible following an approach similar to the estimations of the blackbody shift in Ref. [77].

We estimate very conservatively, that the radio frequency cavity used to drive the discharge scatters about $10 \mathrm{~W}$ into an area of $10 \times 10 \mathrm{~cm}^{2}$ resulting in an intensity of $I=1000 \mathrm{~W} / \mathrm{m}^{2}$. With $E^{2}=2 \eta I$ and $\eta=376 \Omega$ we calculate the electric field to be

$$
E_{\text {outside }}^{2}=\left(850 \frac{\mathrm{V}}{\mathrm{m}}\right)^{2} \text {. }
$$

With an antenna connected to a spectrum analyzer we measure the attenuation by the vacuum chamber to be $10^{-4}$ (see Figure 5.7). Using this attenuation and assuming a similar damping of $10^{-3}$ due to the enclosure of the differential pumping region we estimate the electric field strength within the excitation region to

$$
E_{\text {interaction }}^{2}=\left(3 \frac{\mathrm{mV}}{\mathrm{cm}}\right)^{2} \text {. }
$$

It is hard to think of any mechanism that could shift the $1 S$ level due to the electric field calculated in Eq. (5.11). Comparison with the blackbody radiation with its several orders of magnitude higher electric fields suggests that there is no significant shift. 


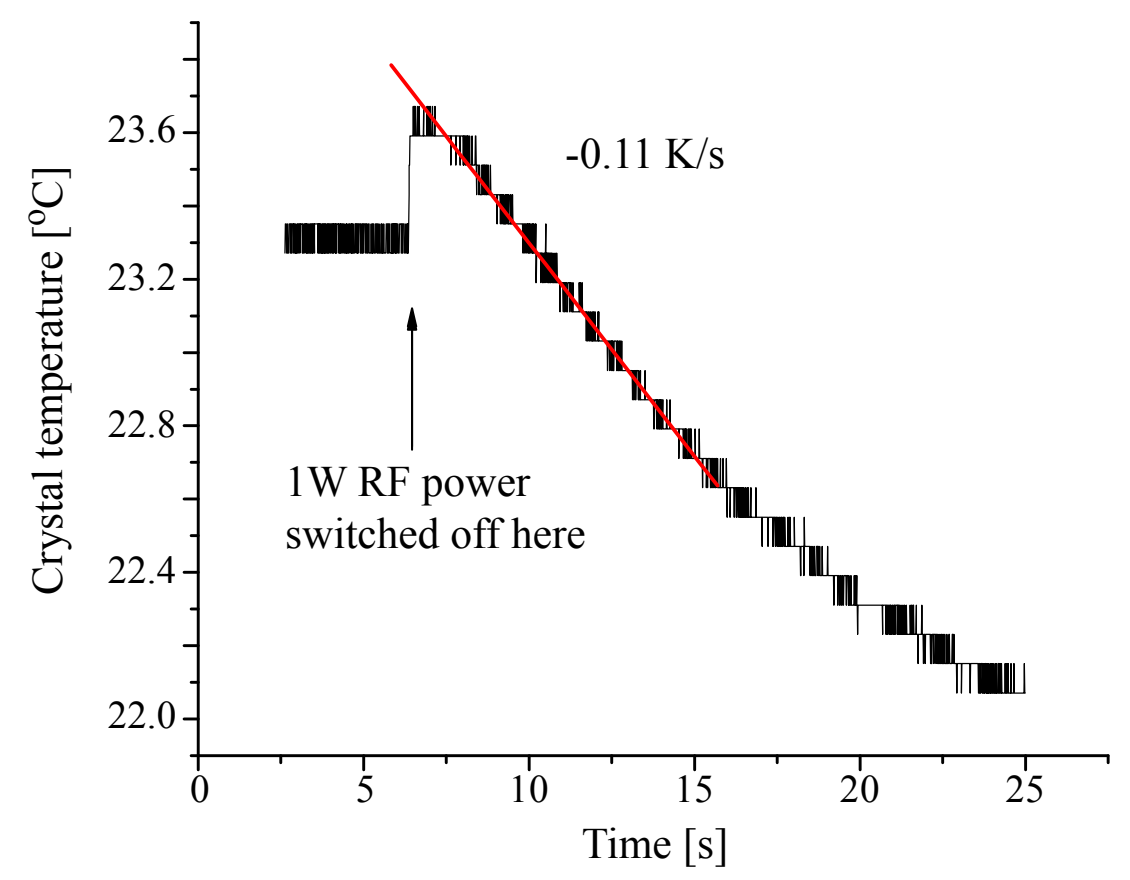

Figure 5.8: Temperature dependence of the power-switching AOM. After switching off $1 \mathrm{~W}$ of radio-frequency power fed to the AOM, the $8 \mathrm{~mm}$ long crystal cools with $-0.11 \mathrm{~K} / \mathrm{s}$. The associated frequency shift is $\Delta f_{\mathrm{AOM}} / f_{1 S-2 S}=1.1 \times 10^{-16}$.

The influence on the $2 S$ can be estimated using the dc Stark shift coefficient ${ }^{\dagger}$. We thus calculate a shift of $\Delta f_{\mathrm{rf}}=(3 \mathrm{mV} / \mathrm{cm})^{2} \cdot 3600 \mathrm{~Hz} /(\mathrm{V} / \mathrm{cm})^{2}=0.03 \mathrm{~Hz}$ which is

$$
\Delta f_{\mathrm{rf}} / f_{1 S-2 S}=1.2 \times 10^{-17}
$$

and therefore negligible at the current level of accuracy.

\subsubsection{Frequency Shift due to the Power-Switching Acousto-optic Modulator}

The frequency shift caused by the AOM operated in the $243 \mathrm{~nm}$ beam path to quickly alter the intensity level (see Figure 3.4, AOM 2) shall be investigated here. Since the power level change is accomplished by switching the driving radio frequency (rf) on and off, temperature fluctuations in the AOM can cause a frequency shift. To characterize the temperature change due to the applied rf we directly attach an AD590 temperature sensor to the AOM crystal. Then, we apply $1 \mathrm{~W}$ of $\mathrm{rf}$ power and wait until the crystal reaches its thermal equilibrium. After switching off the rf source, we monitor the temperature change

\footnotetext{
${ }^{\dagger}$ The $2 S_{1 / 2}$ and $2 P_{1 / 2}$ level are split by $\sim 1 \mathrm{GHz}$. Thus, the $2.4 \mathrm{GHz}$ microwave field is similarly detuned from this transition as a dc field at $0 \mathrm{~Hz}$.
} 
in the crystal (see Figure 5.8). For times of about $10 \mathrm{~s}$ we can approximate the exponential decay with a straight line and find a temperature change of $0.1 \mathrm{~K} / \mathrm{s}$. Two possible shift mechanisms come to mind: a Doppler shift due to a length change and a frequency chirp due to the change of refractive index.

With a thermal expansion coefficient of $0.5 \times 10^{-6} / \mathrm{K}$ and a crystal length of $l=8 \mathrm{~mm}$, the Doppler effect due to a length change for a temperature change rate of $0.1 \mathrm{~K} / \mathrm{s}$ is only $1 \times 10^{-18}$ and thus negligible at the current level of accuracy.

The refractive index's thermal dependence for fused silica at $243 \mathrm{~nm}$ is $\Delta n / \Delta T=$ $2 \times 10^{-5} \mathrm{~K}^{-1}$. The frequency shift at $121 \mathrm{~nm}$ holds

$$
\Delta f=\frac{\Delta n}{\Delta T} \cdot \frac{\Delta T_{\mathrm{AOM}}}{\tau} \cdot \frac{4 l}{\lambda}
$$

with $l=8 \mathrm{~mm}$ and $\lambda=243 \mathrm{~nm}$ we get $0.26 \mathrm{~Hz}$ which is - expressed in fractional frequency units -

$$
\Delta f_{\mathrm{AOM}} / f_{1 S-2 S}=1.1 \times 10^{-16}
$$

and thus negligible at the current level of accuracy.

\subsubsection{Line Pulling by the $\mathrm{m}_{\mathrm{F}}=0$ Hyperfine Component}

Considering the $1 S-2 S$ transition in atomic hydrogen, let $f(1, \pm 1)$ be the hyperfine transitions $F=1, m_{F}= \pm 1 \rightarrow F=1, m_{F}= \pm 1$ and $f(1,0) F=1, m_{F}=0 \rightarrow F=1, m_{F}=0$ (Section 2.3). Then,

$$
f(1,0)-f(1, \pm 1)=9600 \mathrm{~Hz} / \mathrm{G}^{2} .
$$

In the experiment we apply a magnetic bias field of $5 \mathrm{G}$ to separate the transitions. Here, it shall be estimated by how much $f(1, \pm 1)$ can be shifted by the far wing of the $f(1,0)$ transition.

Let $g(\nu)$ be the line shape of $f(1, \pm 1)$ with $\nu$ being the frequency in units of the transition line width $\gamma \approx 2 \mathrm{kHz}$. Separating $g(\nu)$ into an unperturbed contribution and a contribution due to $f(1,0)$ we can write

$$
g(\nu)=\frac{1}{\gamma+4 \nu^{2}}+\frac{a}{\gamma+4\left(\nu-\nu_{0}\right)^{2}}
$$

with the relative amplitude $a$ and the frequency separation of the two transitions $\nu_{0}$. The center frequency of $g(\nu)$ is its maximum, consequently we are looking for $\partial g / \partial \nu=0$. Thus,

$$
\frac{\partial g}{\partial \nu}=\frac{-8 \nu}{\left(\gamma+4 \nu^{2}\right)^{2}}-\frac{-8 a\left(\nu-\nu_{0}\right)}{\left(\gamma+4\left(\nu-\nu_{0}\right)^{2}\right)^{2}}=0
$$

and with $\nu \ll \gamma$ and $\nu_{0} \gg \gamma$ we have

$$
\nu=-\frac{a}{16 \nu_{0}^{3}} \cdot \gamma^{2} .
$$


With a homogeneous magnetic field of $5 \mathrm{G}, \nu_{0}=9600 \cdot 25 \mathrm{~Hz} / \gamma$ and $a=1 / 2^{\ddagger}$ we find $\nu=6 \times 10^{-8} \gamma^{2}$, meaning $\Delta f_{\mathrm{lp}}=-3.6 \mathrm{mHz}$ and thus

$$
\Delta f_{\mathrm{lp}} / f_{1 S-2 S}=-1.5 \times 10^{-18},
$$

which is negligible at our present level of accuracy.

\subsubsection{Higher Order Modes within the Enhancement Cavity}

Due to insufficient mode matching not all light is coupled to the enhancement cavity's $\mathrm{TEM}_{00}$ mode. Instead, a fraction of the light, $I_{2}=I_{0}-I_{\mathrm{TEM}_{00}}$, is not coupled to the cavity but transmitted through its input coupler. We measured the coupling efficiency $I_{\mathrm{TEM}_{00}} / I_{0}>75 \%$ and thus $I_{\mathrm{TEM}_{00}} /\left(I_{0}-I_{\mathrm{TEM}_{00}}\right) \approx 3$.

$I_{\mathrm{TEM}_{00}}$ is amplified in the cavity (Finess $\mathcal{F}=120$ ) to $I_{\mathrm{TEM}_{00}}^{\prime}=I_{\mathrm{TEM}_{00}} \mathcal{F} / \pi$, while $I_{2}$ will be suppressed by the cavity's incoupling mirror transmission $T=0.015$ to $I_{2}^{\prime}=T \cdot I_{2}$. We can already see that $I_{\mathrm{TEM}_{00}}^{\prime} \gg I_{2}^{\prime}$. Consequently, we assume that the light field of the higher order mode contributes only one photon in the two-photon excitation and we get the amplitude ratio

$$
a:=\frac{I_{2}^{\prime} \cdot I_{\mathrm{TEM}_{00}}^{\prime}}{\left(I_{\mathrm{TEM}_{00}}^{\prime}\right)^{2}}=\frac{I_{2}^{\prime}}{I_{\mathrm{TEM}_{00}}^{\prime}}=\frac{T \cdot I_{2}}{I_{\mathrm{TEM}_{00}} \mathcal{F} / \pi}=\frac{\pi T}{3 \mathcal{F}}=1.2 \times 10^{-4} .
$$

We estimate a shift of the unperturbed line (absorption of two photons with $\left(\vec{k}_{1},-\vec{k}_{1}\right)$ ) by line pulling by the Doppler shifted component $\left.\left(\vec{k}_{1},-\vec{k}_{2}\right)\right)$, with $\vec{k}_{1}$ being the $k$-vector belonging to $I_{\mathrm{TEM}_{00}}^{\prime}$ and and $\vec{k}_{2}$ that of $I_{2}^{\prime}$, respectively. The line pulling effect can be estimated by generalizing Eq. (5.17) for two spectral lines with different line widths $\gamma_{1}$ and $\gamma_{2}$

$$
\Delta \nu=-\left(\frac{\gamma_{1}}{\gamma_{2}}\right)^{2} \cdot a \cdot \frac{\nu_{0}}{\left(1+4 \nu_{0}^{2} / \gamma_{2}^{2}\right)^{2}} .
$$

The frequency shift due to the longitudinal and transversal first order Doppler effect is

$$
\Delta \nu_{\mathrm{dp}, \mathrm{L}}=\frac{v_{\mathrm{L}}}{\lambda} \frac{\alpha^{2}}{2} \quad \text { and } \quad \Delta \nu_{\mathrm{dp}, \mathrm{T}}=\frac{v_{\mathrm{T}}}{\lambda} \frac{\alpha^{2}}{2}
$$

with a laser beam divergence of $\alpha=10^{-3} \mathrm{rad}$ and velocities $v_{\mathrm{L}}=150 \mathrm{~m} / \mathrm{s}$ and $v_{\mathrm{T}}=1.5 \mathrm{~m} / \mathrm{s}$ for delay $610 \mu$ s we calculate

$$
\Delta \nu_{\mathrm{dp}, \mathrm{L}}=620 \mathrm{~Hz} \quad \text { and } \quad \Delta \nu_{\mathrm{dp}, \mathrm{T}}=12 \mathrm{kHz} .
$$

Assuming a velocity spread equal to the central velocity, we use Eq. (5.21) with $\Delta \nu_{\mathrm{dp}, \mathrm{L} / \mathrm{T}}=$ $\nu_{0}=\gamma_{2}$ and $\gamma_{1}=2 \mathrm{kHz}$ to calculate the line pulling effect by the second, Doppler shifted resonance as

$$
\Delta f_{\mathrm{L}}=0.032 \mathrm{~Hz} \quad \text { and } \quad \Delta f_{\mathrm{T}}=0.0015 \mathrm{~Hz} .
$$

${ }^{\ddagger}$ This represents a rather conservative estimate given the laser being in resonance with $f(1, \pm 1)$ and thus far detuned from $f(1,0)$ 


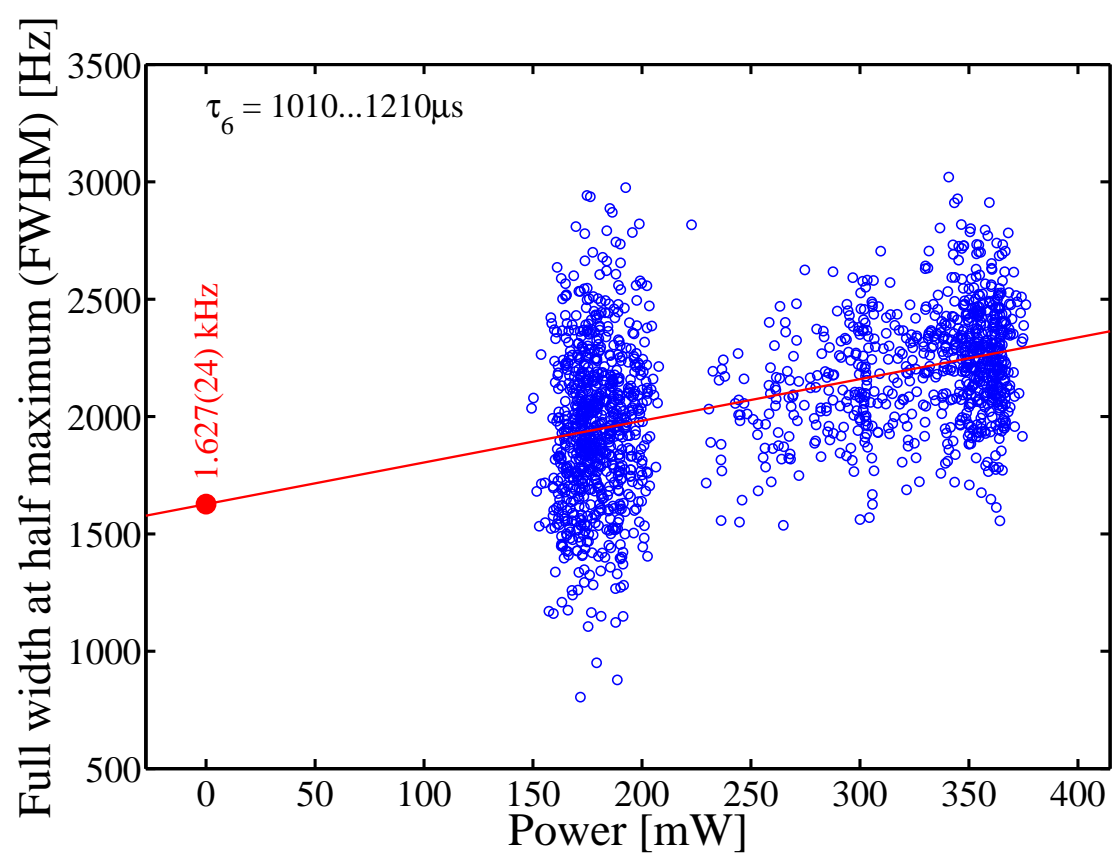

Figure 5.9: Full width at half maximum of the fitted Lorentzian versus intracavity power for delay $\tau_{6}=1010 \ldots 1210 \mu$ s and extrapolation to zero power. The experimentally observed line width does not agree with simulation as discussed in Appendix C.2.1.

Expressing these effects in fractional frequency units

$$
\Delta f_{\mathrm{L}} / f_{1 S-2 S}=1.3 \times 10^{-17} \quad \text { and } \quad \Delta f_{\mathrm{T}} / f_{1 S-2 S}=6.1 \times 10^{-19},
$$

we see that the frequency shifts associated with insufficient mode matching to the enhancement cavity are negligible at the current level of accuracy.

\subsubsection{Recoil Shift}

In his thesis [79], A. Huber discusses a possible shift of the $1 S-2 S$ transition due to the finite localization of the standing wave within the enhancement cavity which he restricts at the $4 \times 10^{-15}$ level. Originally raised by Christian Bordé, the effect was later discussed in the literature [80]. Here, we want to show that the effect is negligible at the current level of accuracy.

Due to the localization of the photons in the enhancement cavity, their $k$-vectors (momenta) need to have a spread due to Heisenberg's uncertainty principle. With this momentum spread it is not guaranteed that always two anti-collinear photons are absorbed resulting in a first order Doppler as well as a recoil shift. While the first order Doppler shift should average to zero due to the cavity symmetry and thus only cause a broadening of the 
spectral line, the recoil shift will always have a positive sign. We can use the line width observed in the $1 S-2 S$ experiment, extrapolated zero power, to restrict the first order Doppler effect and thus the angle $\alpha$ between two non-anti-collitiar photon momenta. Considering only small angles $\alpha \ll 1$, it becomes evident that a possible shift would mainly be caused by a transverse velocity $v_{\mathrm{T}} \propto \alpha$ while the effect on the longitudinal velocity $v_{\mathrm{L}} \propto \alpha^{2} / 2$ is even smaller. ${ }^{\S}$ In Figure 5.9 we see that

$$
\left.\gamma\right|_{P=0}=1627(24) \mathrm{Hz} \text {. }
$$

The first order Doppler effect holds $\Delta \nu / \nu_{1 S-2 S}=v_{\mathrm{T}} / c$ with the transverse velocity $v_{\mathrm{T}}=$ $\hbar k \alpha / m_{\mathrm{H}}=h \alpha /\left(\lambda m_{\mathrm{H}}\right)$. Thus,

$$
\alpha=\frac{\Delta \nu \lambda m_{\mathrm{H}} c}{\nu_{1 S-2 S} h}=1.2 \times 10^{-4} \mathrm{rad} .
$$

\footnotetext{
${ }^{\S} \cos (\alpha)=1+\alpha^{2} / 2+\ldots$ The first term in the Taylor expansion represents strictly anti-collinear photon-momenta resulting in no shift.
}

Table 5.1: Uncertainty budget for $f_{1 S-2 S} \approx 2.466 \times 10^{15} \mathrm{~Hz}$. For delay dependent corrections (e.g. second order Doppler effect) the stated correction is the correction to the final value, i.e. the weighted average of the corrections for delays $\tau_{5,6}$.

\begin{tabular}{l|r|r|r} 
& correction & uncertainty & rel. uncertainty \\
& {$[\mathrm{Hz}]$} & {$[\mathrm{Hz}]$} & {$\left[10^{-15}\right]$} \\
\hline statistics & - & 6.3 & 2.6 \\
2nd order Doppler effect & +52.6 & 5.1 & 2.0 \\
line shape model & - & 5.0 & 2.0 \\
quadratic ac Stark shift $(243 \mathrm{~nm})$ & -15.9 & 2.0 & 0.8 \\
ac Stark shift, $486 \mathrm{~nm}$ quench light & - & 2.0 & 0.8 \\
hyperfine correction & +310 712 & 229.4 & 1.7 \\
dc Stark effect & - & 1.0 & 0.69 \\
ac Stark shift, 486 nm scattered light & - & 1.0 & 0.4 \\
Zeeman shift & - & 0.93 & 0.4 \\
pressure shift & - & 0.5 & 0.38 \\
blackbody radiation shift & +1.0 & 0.3 & 0.2 \\
power modulation AOM chirp & - & 0.3 & 0.12 \\
rf discharge ac Stark shift & - & 0.03 & 0.11 \\
higher order modes & - & 0.03 & 0.012 \\
line pulling by $m_{F}=0$ component & - & 0.004 & 0.012 \\
recoil shift & - & 0.009 & 0.0016 \\
FOM & -25.9 & 2.0 & 0.0036 \\
gravitational red shift & - & 0.04 & 0.81 \\
\hline total & & 10.4 & 0.077 \\
\hline
\end{tabular}


With this we find

$$
\Delta \nu_{\text {recoil }}=\frac{\left(\Delta p_{\mathrm{T}}\right)^{2}}{2 m_{\mathrm{H}} h}=\frac{\alpha^{2} h}{\lambda^{2} 2 m_{\mathrm{H}}}=49 \mathrm{mHz} \quad(\text { at } 243 \mathrm{~nm})
$$

This results in a possible recoil shift at $121 \mathrm{~nm}$ of $98 \mathrm{mH}$ or

$$
\Delta f_{\text {recoil }} / f_{1 S-2 S}=4.0 \times 10^{-17}
$$

\subsection{Result}

Summarizing all corrections and uncertainties (Table 5.1) we find the $1 S-2 S$ hyperfine centroid frequency to be

$$
f_{1 S-2 S}=2466061413187035(10) \mathrm{Hz}
$$

which confirms the previous best measurement [3] but is 3.3 times more accurate. A comparison of the previous two measurements and the current result is presented in Figure 5.10. It is evident that the excessive day to day scatter present in the 1999 and 2003 measurement has been removed in the current measurement. We have identified the origin

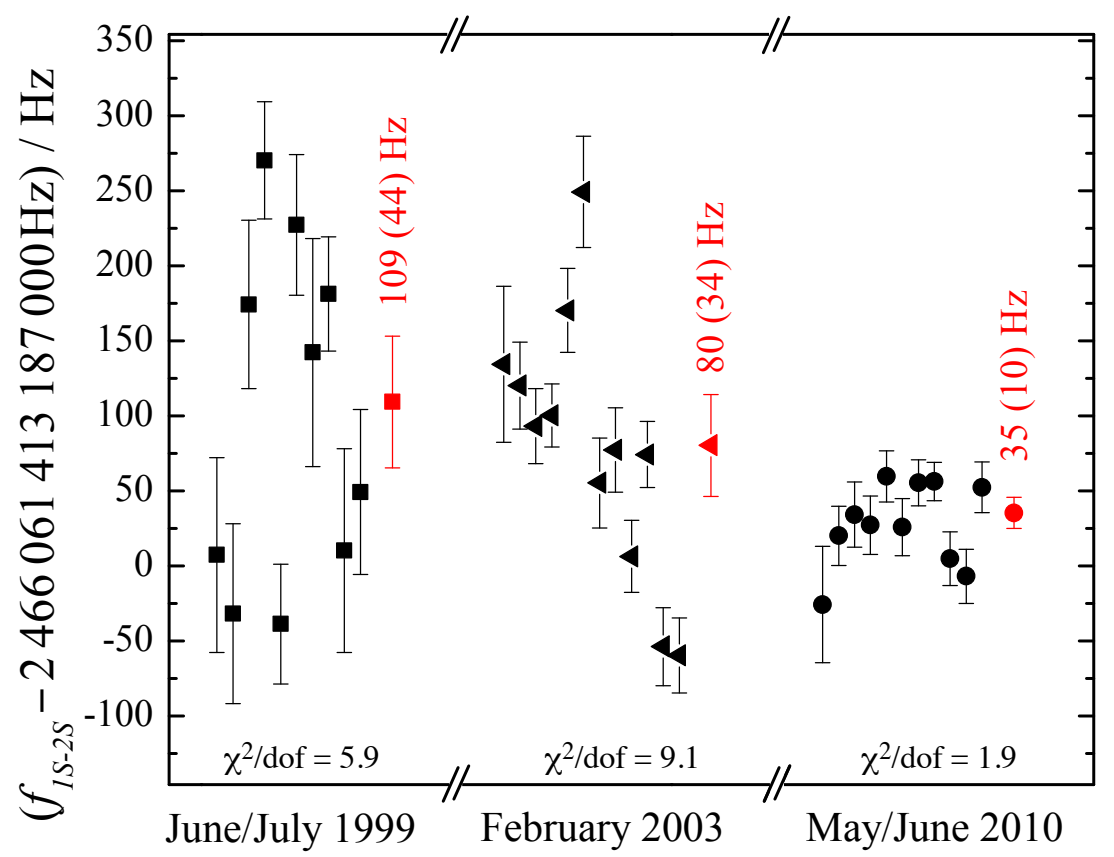

Figure 5.10: The $1 S-2 S$ centroid frequency of the current measurement is compared with the previous two measurements $[13,3]$. The black points represent an average per day, the red points (with label) are the final values with the error bar representing the $1 \sigma$ uncertainty. 
of the excess scatter as insufficient low frequency gain of the feedback loop of the dye laser. This effect has become apparent only after comparing with a second, independent cavity. A more detailed discussion of the previous problem is given in Appendix D. 


\section{Chapter 6}

\section{Second Absolute Frequency Measurement}

\subsection{Introduction}

Following a proposal by Brett Altschul [72] we decided to measure the $1 S-2 S$ transition for a second time. With the measurement taking place six months after the measurement in May 2010, we were able to maximize the sensitivity of a possible Lorentz boost symmetry violation which can be characterized within the standard model extensions (SME) introduced by Kostelecký and coworkers [15, 81].

Also, the measurement presents the first application of the long haul optical fiber link between Physikalisch-Technische Bundesanstalt (PTB) in Braunschweig and MPQ (Section 3.3.3).

\subsection{Experimental Summary}

\subsubsection{Laser System and Frequency Calibration via the $900 \mathrm{~km}$ Fiber Link}

Again, the $1 S-2 S$ transition is excited with the forth harmonic of ECDL1. The frequency comb setup referenced to the hydrogen maser is identical to the measurement in May 2010 (Section 5). In contrast to any previous measurement, there was no primary frequency reference present at MPQ. Instead, the maser was calibrated using the remote cesium fountain atomic clock at PTB made available at MPQ via an optical fiber link (compare Section 3.3.3). 

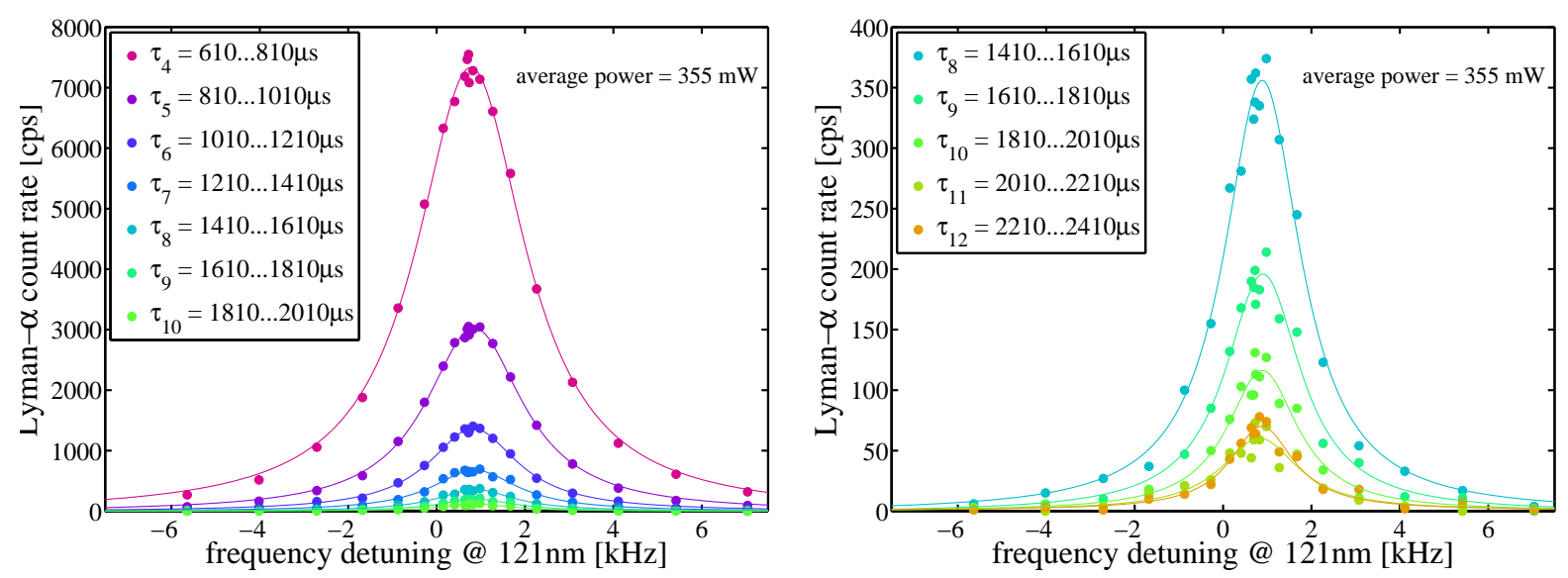

Figure 6.1: Central part of the $1 S-2 S$ resonance profiles recorded with the high efficiency detector, along with a Lorentzian fit. Although delays up to $\tau_{12}=2210 \ldots 2410 \mu \mathrm{s}$ are detectable they are not suited for analysis because the very slow atoms have mostly seen light during the enhancement-cavity settling time (Section 3.1.3). Note the different scales of the vertical axes.

\subsubsection{Statistical Improvement with the High-Efficiency Lyman- $\alpha$ De- tector}

The high-efficiency detector (Section 3.1.2) improves the typical count rate by a factor of 20. Typical line profiles are shown in Figure 6.1. While with the old photo multiplier tube delay $\tau_{8}=1410 \ldots 1610 \mu \mathrm{s}$ was the highest resolvable delay, with the new detector delays all the way up to $\tau_{12}=2210 \ldots 2410 \mu$ s are visible. However, for the analysis we will only consider delays $\tau_{5,6}$. Higher delays are prone to the settling in the enhancement cavity lock as described in Section 3.1.3.

A comparison of data obtained with both old and new detector is shown in Figure 6.2. The improved count rate resulting from the increased detection efficiency manifests itself in improved statistics. This means that the statistical part of the experiment is not limited by hidden systematic effects and one can hope to further improve the accuracy.

\subsubsection{Measurement Process}

For the second absolute frequency measurement, the measurement procedure was kept very similar to that in May (Section 5.2.3). However, for reasons not entirely clear but almost certainly connected with the hydrogen beam, the count rate with the Lyman- $\alpha$ PMT detector was poor. After a week of measurement with this poor statistics, we decided to install the high-efficiency Lyman- $\alpha$ detector (Figure 6.2, run 26). Although not thoroughly tested, it promised very good statistics. For the final analysis, we only use the data obtained 


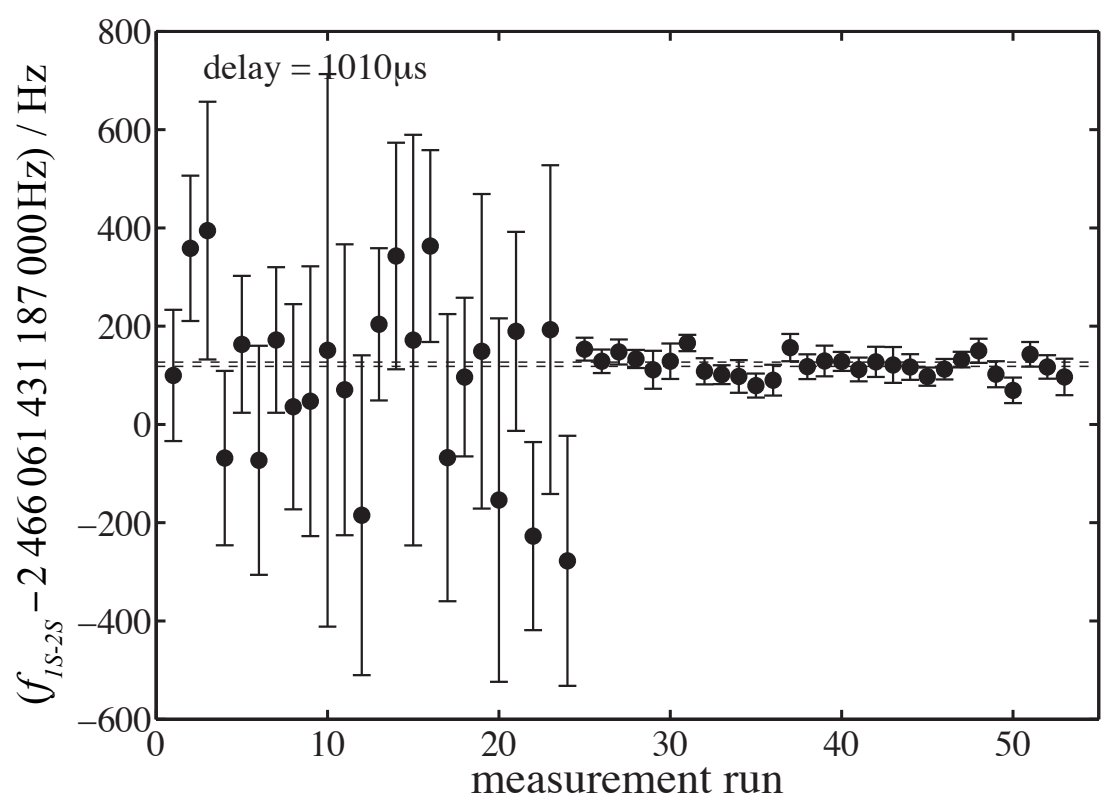

Figure 6.2: Shown is the $1 S-2 S$ frequency plotted versus freezing cycle. The new detector was installed during cycles 24 and 25. The increased count rate directly improves the statistics. The MPQ maser is used as reference for this data.

from the new, high-efficiency Lyman- $\alpha$ detector for the following reason. During the first week, the data format at PTB which is needed for the frequency calibration of the MPQ maser was changed several times. So far, nobody has been able to work out the analysis manually. However, from Figure 6.2 with all data referenced to the maser, one concludes that the results from both detectors are compatible.

Again (Section 5.2.3), the mirrors of the enhancement cavity degraded during the course of a measurement day, mostly after melting the hydrogen ice from the nozzle. Thus, we opened the chamber in the mornings, cleaned the mirrors with methanol and had the cryopump running by 10 a.m. On average, the available uv power for spectroscopy was a factor of 1.5 smaller than in the first measurement in May 2010. The new detector over compensated the associated loss in count rate. Working with lower power gave an independent check on the efficient compensation of the quadratic ac Stark effect (Section 5.3.1).

622 spectral lines have been recorded during five days of measurement from November 22 to $26,2011$.

\subsection{Summary of Uncertainties}

The data evaluation follows that of Section 5 without modifications. Using the same procedure on two different data sets allows to test the reliability of the procedure (Section 6.4.1).

A summary of the contributing uncertainties can be found in Table 6.1. It should 
6.4. RESULT

be noted that the statistical uncertainty is smaller than during the absolute frequency measurement in May (Section 5) due to the higher detection efficiency in this measurement.

\subsubsection{Additional Uncertainty of the Second Order Doppler Effect with the New Lyman- $\alpha$ Detector}

On the other hand, we have to increase the uncertainty due to the second order Doppler effect. After the measurement campaign it was discovered that the pressure in the detector region is increased with the new detector. The newly introduced Faraday cage (Section 3.1.2) reduces the pumping speed. The increased pressure leads to collisional quenching which blurs the otherwise well localized quench region. This leads to an additional uncertainty in the velocity distribution for each delay $\tau$ which can be translated to a higher uncertainty in the second order Doppler effect using Monte Carlo simulation. The increased pressure does not create an additional pressure shift: Atoms contributing to high delays are mostly excited at the beginning of the excitation region, consequently, they don't experience the high pressure during excitation.

\subsection{Result}

During the November measurement we find the absolute frequency of the $1 S-2 S$ transition in atomic hydrogen to be

$$
f_{1 S-2 S}=2466061413187017(11) \mathrm{Hz} .
$$

This corresponds to a fractional frequency uncertainty of $4.4 \times 10^{-15}$ and is in excellent agreement with the May measurement as can be seen in Figure 6.3.

\subsubsection{Agreement of both Absolute Frequency Measurements}

Both absolute frequency measurements agree:

$$
\Delta f_{1 S-2 S}=f_{1 S-2 S}^{\text {may }}-f_{1 S-2 S}^{\text {nov }}=18(15) \mathrm{Hz}
$$

The agreement confirms the confidence in the determined absolute frequency for a number of reasons. First, although the evaluation procedure is the same for both measurements, the experimental conditions where different for the two experiments. While in May, the excitation power was chosen between $150 \mathrm{~mW}$ and $350 \mathrm{~mW}$, in November it was more between $100 \mathrm{~mW}$ and $250 \mathrm{~mW}$. The unperturbed frequency is independent on excitation power levels present in the experiment suggesting that the correction of both the linear and quadratic ac Stark shift are well under control (compare Figure 6.4).

Second, the velocity distribution measurement was performed approximately half way between the two measurement campaigns. Consequently, the evaluation heavily relies 
Table 6.1: Uncertainty budget for $f_{1 S-2 S} \approx 2.466 \times 10^{15} \mathrm{~Hz}$ during the second measurement in November 2010. For delay dependent corrections (e.g. second order Doppler effect) the stated correction is the correction to the final value, i.e. the weighted average of the corrections for delay $\tau_{5,6}$.

\begin{tabular}{l|r|r|r} 
& correction & uncertainty & rel. uncertainty \\
& {$[\mathrm{Hz}]$} & {$[\mathrm{Hz}]$} & {$\left[10^{-15}\right]$} \\
\hline statistics & - & 3.3 & 1.3 \\
2nd order Doppler effect & +34.2 & 8.0 & 3.2 \\
line shape model & - & 5.0 & 2.0 \\
quadratic ac Stark shift $(243 \mathrm{~nm})$ & -10.7 & 2.0 & 0.8 \\
ac Stark shift, $486 \mathrm{~nm}$ quench light & - & 2.0 & 0.8 \\
hyperfine correction & 71229.4 & 1.7 & 0.69 \\
dc Stark effect & - & 1.0 & 0.4 \\
ac Stark shift, 486 nm scattered light & - & 1.0 & 0.4 \\
Zeeman shift & - & 0.93 & 0.38 \\
pressure shift & - & 0.5 & 0.2 \\
blackbody radiation shift & +1.0 & 0.3 & 0.12 \\
power modulation AOM chirp & - & 0.3 & 0.11 \\
rf discharge ac Stark shift & - & 0.03 & 0.012 \\
higher order modes & - & 0.03 & 0.012 \\
line pulling by $m_{F}=0$ component & - & 0.004 & 0.0016 \\
recoil shift & - & 0.009 & 0.0036 \\
CSF1 & +91.2 & 1.9 & 0.76 \\
gravitational red shift & -130.0 & 0.04 & 0.077 \\
\hline total & & 10.8 & 4.4
\end{tabular}

on the stability of this distribution over the course of a year. Using the same velocity distributions to correct both measurements and still obtaining the same value suggests that the assumption of a stable velocity distribution is correct.

Third, the frequency reference was completely different with a much more complicated setup in the November measurement. For this measurement, a lot of additional laser frequency comb beat notes needed to be counted. Independent of the many individual tests of our cycle slip criteria, the agreement of the measurements proves the correctness of the frequency measurement.

In the following two sections, the two measurements shall be used to test fundamental theories. 


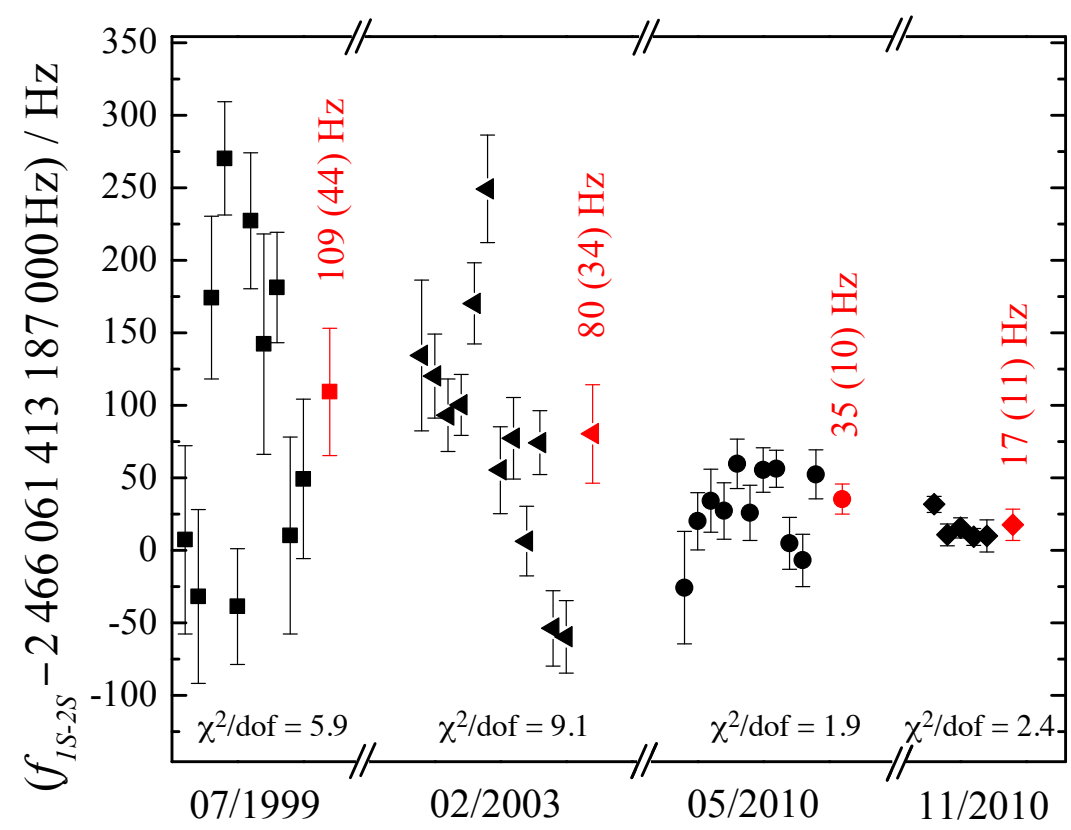

Figure 6.3: Comparison of May and November 2010 measurement along with the previous two best measurements $[13,3]$. Each data point represents the average of one day of data while the red data points (with label) are the weighted mean for each measurement campaign.

\subsection{Analysis of the Hydrogen Experiment within the Stan- dard Model Extension (SME) Framework}

\subsubsection{Introduction to SME}

As part of the quest for a fundamental physical theory unifying quantum mechanics and the theory of gravitation, the search for physics beyond the standard model of particle physics is extremely important. Many candidate theories for quantum gravity, such as string theory [82] or loop quantum gravity [83, 84], may allow violations of Lorentz invariance and charge conjugation / parity / time reversal (CPT) invariance.

The minimal standard model extension (SME) introduced by Kostelecký and coworkers $[81,15]$ offers a systematic way of parameterizing such violations. Testing Lorentz and CPT violations within the SME framework means constraining these parameters from astrophysical, accelerator, or non-relativistic laboratory measurements. 

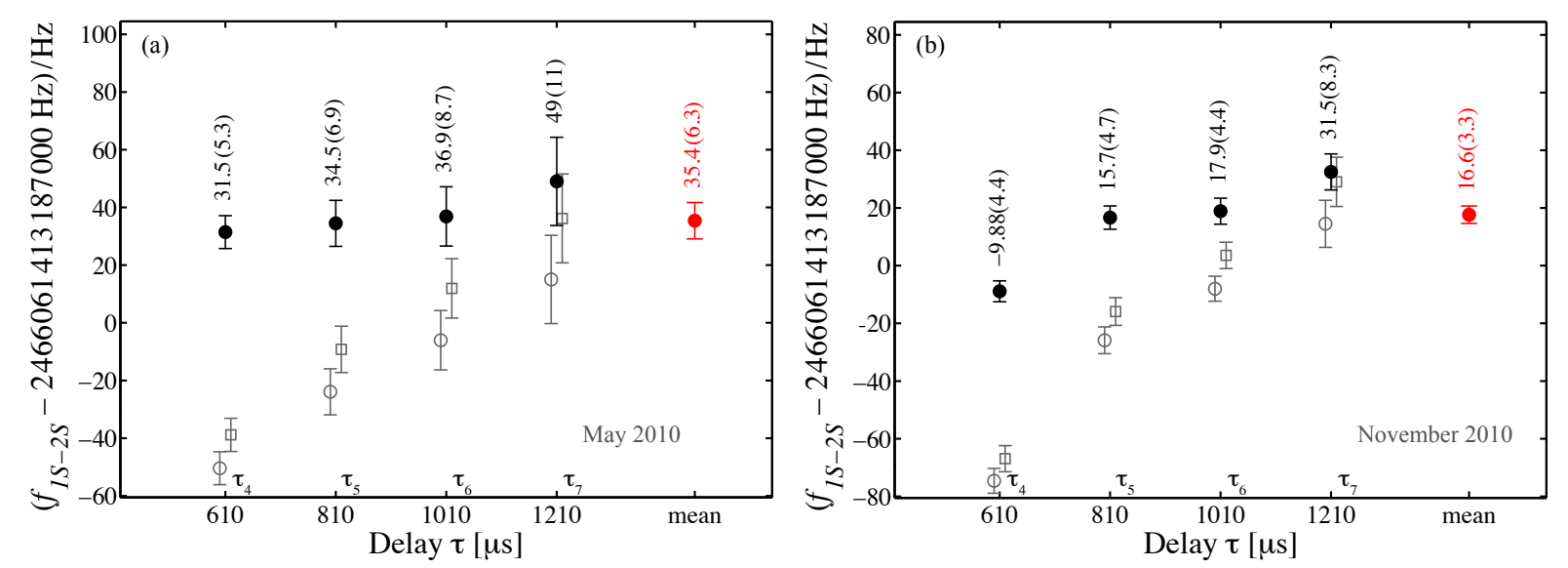

Figure 6.4: The plot shows $f_{1 S-2 S}$ with all corrections applied (compare Figure 5.5). The error bars represent the $1 \sigma$ statistical uncertainty. The open circles show the same data with the second order Doppler effect not corrected, the open squares do neither include the second oder Doppler effect nor the quadratic ac Stark shift corrections. The mean is calculated from delays $\tau_{5,6}$. (a) The same correction work for the May 2010 data and the (b) November 2010 data.

\subsubsection{The SME Coordinate System}

The Standard Model Extensions use a sun-centered coordinate system (Figure 6.5) in which the equatorial plane of the earth's orbit is tilted against the $Y$-axis by $\eta \simeq 23.5^{\circ}$ with the $X$-axis being the rotation axis [85].

The earth is orbiting the sun with a velocity $v_{\oplus} \approx 10^{-4} c$ with $c$ being the speed of light. $\quad \vec{v}_{\oplus}$ can be expressed in terms of the orbital angular frequency $\Omega_{\oplus}$ and the time coordinate $T$ which is measured relative to the vernal equinox in the year $2000 \vec{v}_{\oplus}=$ $v_{\oplus}\left(\sin \Omega_{\oplus} T,-\cos \eta \cos \Omega_{\oplus} T,-\sin \eta \cos \Omega_{\oplus} T\right)$.

\subsubsection{Using the Hydrogen Experiment to Test Lorentz Boost Invari- ance}

The Lagrange density of the SME includes operators that can be constructed from standard model fields, contracted with Lorentz violating background tensors*. The background tensor that can be constrained with results obtained from $1 S-2 S$ spectroscopy of hydrogen is the two-index tensor $c^{\nu \mu}$ which breaks Lorentz invariance but not CPT [72]. The hydrogen electronic structure, and thus the $1 S-2 S$ frequency, dependance on the $c^{\nu \mu}$ coefficients. Because the dependence changes with different points of the Earth's orbit around the sun, two or more measurements of the $1 S-2 S$ frequency at different times are sensitive to the boost invariance violation parameters $c_{(T J)}$.

\footnotetext{
*A CPT violating tensor necessarily violates Lorentz symmetry.
} 
The nonrelativistic Hamiltonian for the electron in the hydrogen atom is

$$
H=\frac{p_{j} p_{k}}{2 m}\left[\delta_{j k}-c_{00} \delta_{j k}-c_{(j k)}\right]-c_{(0 j)} p_{j}-\frac{\alpha \hbar c}{r}
$$

to first order in $c^{\nu \mu}$. Because we are looking for a small effect, it is sufficient to only consider the leading order energy shifts, calculated from the nonrelativistic Schrödinger equation. We find

$$
\frac{\Delta E_{n}}{h}=-\frac{m c^{2} \alpha^{2}}{2 n^{2}}\left(c_{00}+\frac{2}{3} c_{j j}\right)
$$

with the principal quantum number $n$ and the $c_{00}$ and $c_{j j}$ coefficients being defined in the laboratory frame.

With the laboratory moving with the earth which orbits the sun with a velocity $\vec{v}_{\oplus}$, the laboratory frame coefficients relate to the coefficients in the sun-centered frame (compare Section 6.5.2) by $c_{j j} \approx \frac{v_{J}}{c} c_{(T J)}$ and $c_{00} \approx \frac{v_{J}}{c} c_{(T J)}$ where we have neglected terms which cannot violate Lorentz boost invariance.

The $1 S-2 S$ frequency difference measured at two distinct points around the Earth's orbit is

$$
\delta f_{1 S-2 S}=\frac{5}{3} \frac{\delta v_{J}}{c} c_{(T J)} f_{1 S-2 S}, \quad J=x, y, z
$$

where $\delta \vec{v}=\vec{v}_{2}-\vec{v}_{1}$ is the difference in orbital velocities at the two points. When averaging the velocities for the May and November measurements, respectively, we find the result

$$
0.95 c_{(T X)}-0.29 c_{(T Y)}-0.08 c_{(T Z)}=(2.2 \pm 1.8) \times 10^{-11} .
$$

This is an approximately four times stronger bound than placed on a different linear combination of the same coefficient from two earlier hydrogen experiments [72]. This

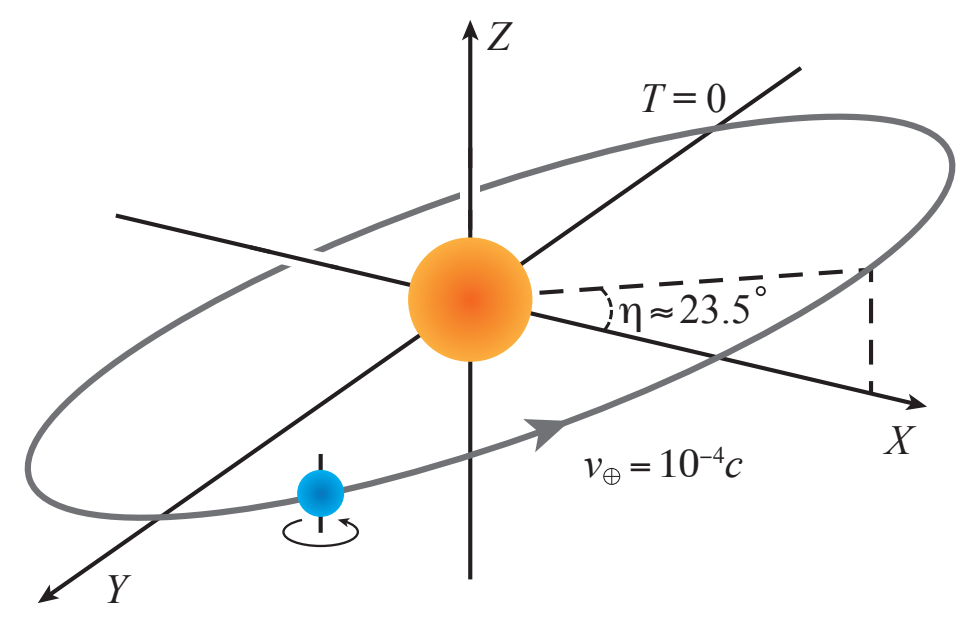

Figure 6.5: The SME uses a sun-centered coordinate system in which the equatorial plane of the earth's orbit is tilted by $\eta \simeq 23.5^{\circ}$ against the $Y$-axis with the $X$-axis being the rotation axis [85]. 
improvement is due to the higher accuracy of the two measurements used for the presented analysis.

\subsubsection{Discussion}

The hydrogen $1 S-2 S$ experiment is a rather peculiar case for determining the $c^{\nu \mu}$ coefficients. First, due to the spherical symmetry of both the $1 S$ and the $2 S$ state, the transition frequency is insensitive to any other SME coefficients except the $c^{\nu \mu}$. Second, as a laser spectroscopy experiment, the test takes place at low energies in contrast to astronomy or accelerator based observations. This is advantageous since at energies on the order of $m^{2} c^{4} / E^{2}$ - close to the energy scale at which those experiments can place limits - new forms of Lorentz violation are expected to become important [86].

Last, the hydrogen $1 S-2 S$ frequency is measured with respect to the Cs ground state hyperfine transition. This implies that the measured transition frequency is not only affected by the coefficients related to the electron but also by those of the proton sector, manifesting themselves in the hyperfine splitting. However, those proton coefficients have been extremely tightly constrained [87], making the $1 S-2 S$ measurement a test of the electron coefficients alone.

A more detailed discussion can be found in [88].

\subsection{Constraint on the Drift of Fundamental Constants}

The current best limit on a possible drift of the fine structure constant $\alpha$ is set by a comparison of transition frequencies in $\mathrm{Hg}^{+}$and $\mathrm{Al}^{+}$[14]. While the hydrogen experiment cannot compete with the uncertainty provided in these experiments which are on the low $10^{-17}$ level it can still provide an independent value for a different time interval. For the analysis, we follow [3].

From the data summarized in Figure 6.3 we deduce a fractional time variation of the frequency ratio $\nu_{\mathrm{Cs}} / \nu_{\mathrm{H}}$ of

$$
\frac{\partial}{\partial t} \ln \left(\nu_{\mathrm{Cs}} / \nu_{\mathrm{H}}\right)=-(3.0 \pm 1.2) \times 10^{-15} \mathrm{yr}^{-1}
$$

during 1999 and 2010. Its sensitivity to a possible variation of the fine structure constant $\alpha$ and the ratio of the cesium magnetic moment to the Bohr magneton is given by [3]

$$
\left.\frac{\partial}{\partial t} \ln \frac{\nu_{\mathrm{Cs}}}{\nu_{\mathrm{H}}}=\frac{\partial}{\partial t}\left(\ln \frac{\mu_{\mathrm{Cs}}}{\mu_{B}}+(2.0+0.8) \ln \alpha\right)\right) .
$$

while a contribution from the quark mass ratio is two orders of magnitude smaller and can be neglected for these considerations.

The time variation of $\alpha$ at our current epoch has been limited to [14]

$$
\frac{\partial}{\partial t} \ln \alpha=(-1.6 \pm 2.3) \times 10^{-17} \mathrm{yr}^{-1}
$$


by optical comparison of the clock transition frequencies of a single $\mathrm{Hg}^{+}$and $\mathrm{Al}^{+}$ion over the course of one year. Combining Equations (6.10) and (6.9) we can put a constraint on the variation of the ratio of the magnetic moments

$$
\frac{\partial}{\partial t} \ln \frac{\mu_{\mathrm{Cs}}}{\mu_{B}}=-(3.0 \pm 1.2) \times 10^{-15} \mathrm{yr}^{-1}
$$

The same constraint can be set using the time variation of the fractional frequency of a single mercury ion $\frac{\partial}{\partial t} \ln \left(\nu_{\mathrm{Cs}} / \nu_{\mathrm{Hg}^{+}}\right)=(3.7 \pm 3.9) \times 10^{-16} \mathrm{yr}^{-1}$ [4]. With this we deduce $\frac{\partial}{\partial t} \ln \frac{\mu_{\mathrm{Cs}}}{\mu_{B}}=(4.7 \pm 4.1) \times 10^{-16} \mathrm{yr}^{-1}$ which is in good agreement with our finding that there is no significant drift of the ratio of magnetic moments. 


\section{Chapter 7}

\section{Conclusion and Outlook}

In this Thesis we have reported on measurements of the $1 S-2 S$ transition in atomic hydrogen and deuterium conducted on a $5.8 \mathrm{~K}$ atomic beam. The transition is excited Dopplerfree via two counter-propagating photons near $243 \mathrm{~nm}$. The excitation laser is stabilized to a Fabry-Perot cavity and its frequency is phase coherently linked to an active hydrogen maser.

\subsection{Hydrogen / Deuterium Isotope Shift}

The hydrogen/deuterium isotope shift, reported on in Section 4, has been determined to

$$
\Delta f_{\exp }=670994334606(15) \mathrm{Hz}
$$

which is a ten-fold improvement over the previous best measurement by Huber et al. [1]. Comparison with the theoretical value for the isotope shift excluding the leading nuclear size effect $\Delta f_{\text {th }}=670999566.90(66)(60) \mathrm{kHz}$, where the first uncertainty is due to experimental constants and the second uncertainty is due to theory, we can extract an rms charge radius difference of deuteron and proton as

$$
\left\langle r^{2}\right\rangle_{d}-\left\langle r^{2}\right\rangle_{p}=3.82007(65) \mathrm{fm}^{2}
$$

a more than twofold improvement compared to the former value.

The inaccuracy of the isotope shift measurement was dominated by a pressure shift, mostly occurring in the high pressure nozzle region. The quench-laser near $486 \mathrm{~nm}$ introduced right in front of the excitation zone (compare Section 3.1.1) reduces this effect by a factor of ten. It also reduces the dc Stark effect, which is among the bigger systematic uncertainties. Thus, with this subtle experimental improvement, a new measurement of the isotope shift should immediately give a better result with a predicted uncertainty of a few hertz.

Unfortunately, an improved isotope shift measurement will not immediately improve the derived rms charge radius difference of deuteron and proton, as theory is limited by 
calculations of the deuteron polarizability. Also, the measurements of the mass ratios of electron to proton and electron to deuteron need to be improved (compare 4.5).

\subsection{S-2S Absolute Frequency}

In Chapter 5, the measurement of the frequency ratio of the $1 S-2 S$ transition in atomic hydrogen to the cesium ground state hyperfine transition provided by the mobile cesium fountain clock FOM is determined to be

$$
f_{1 S-2 S}=2466061413187035(10) \mathrm{Hz}
$$

which presents a fractional frequency uncertainty of $4.2 \times 10^{-15}$ and is a more than three times improvement compared to the previous best measurement by Fischer et al. [3] confirming the previous value.

In Chapter 6, the second absolute frequency measurement of the $1 S-2 S$ transition in atomic hydrogen presents the first application of a $900 \mathrm{~km}$ fiber link between MPQ and Physikalisch-Techniche Bundesanstand (PTB) in Braunschweig which we have used to calibrate the hydrogen maser with the stationary cesium fountain clock CSF1. With the result of

$$
f_{1 S-2 S}=2466061413187017(11) \mathrm{Hz}
$$

we can put a constraint on the electron Lorentz boost violating coefficients

$$
0.95 c_{(T X)}-0.29 c_{(T Y)}-0.08 c_{(T Z)}=(2.2 \pm 1.8) \times 10^{-11}
$$

within the framework of minimal standard model extensions. Also, the drift of the nuclear magnetic moment ratio can be restricted to

$$
\frac{\partial}{\partial t} \ln \frac{\mu_{\mathrm{Cs}}}{\mu_{B}}=-(3.0 \pm 1.2) \times 10^{-15} \mathrm{yr}^{-1}
$$

effectively restricting a drift of the strong coupling constant.

With the establishment of the optical fiber link, it is now much easier to measure the hydrogen frequency at MPQ against a primary cesium standard. Together with new experimental advances this should allow to further improve the absolute frequency of the $1 S-2 S$ transition. First, the newly developed, high-efficiency detector (Section 3.1.2) dramatically improves statistics. From Figure 6.2 one can conclude that the stability of the apparatus has been truly statistics limited, a prerequisite for further improvements. Also, it will allow to work with lower excitation powers to suppress the quadratic ac Stark effect.

To tackle the biggest uncertainty, the second order Doppler effect, one can implement the $486 \mathrm{~nm}$ Doppler laser directly into the detection scheme. Instead of quenching $2 S$ atoms independently of their velocity by a small electric field and then, in a second step, measure the atoms' velocity distribution, one might be able to use the Doppler laser directly for velocity selective detection. The width of the $2 S-4 P$ one-photon transition should allow to 
only address a velocity distribution within a $20 \mathrm{~m} / \mathrm{s}$ width. Working with this more narrow and better defined velocity distribution should improve the control over the second order Doppler effect drastically. Also, it will reduce the observed line width.

At the beginning of this work, the use of an atomic coilgun [89, 90, 91, 92] was considered as a possible source of slow hydrogen atoms. While these devices provide almost arbitrarily slow beams with a narrow velocity distribution, there is a significant drawback to such a system: By design, these devices produce a spin-polarized sample eliminating the cancellation of the Zeeman effect when exciting the $1 S-2 S$ transition (see Section 5.3.8).

Before attempting a new measurement, the chopper frequency needs to be decreased to make use of even higher delays. Also, it should be kept in mind, that the frequency comb system has been proven to be stable on the $5 \times 10^{-16}$ level and accurate to $7 \times 10^{-17}$. As an ultimate test of the fiber link, as needed for an absolute frequency measurement of the $1 S-2 S$ transition in hydrogen, a comparison of CSF1 at PTB and FOM at MPQ would be desirable.

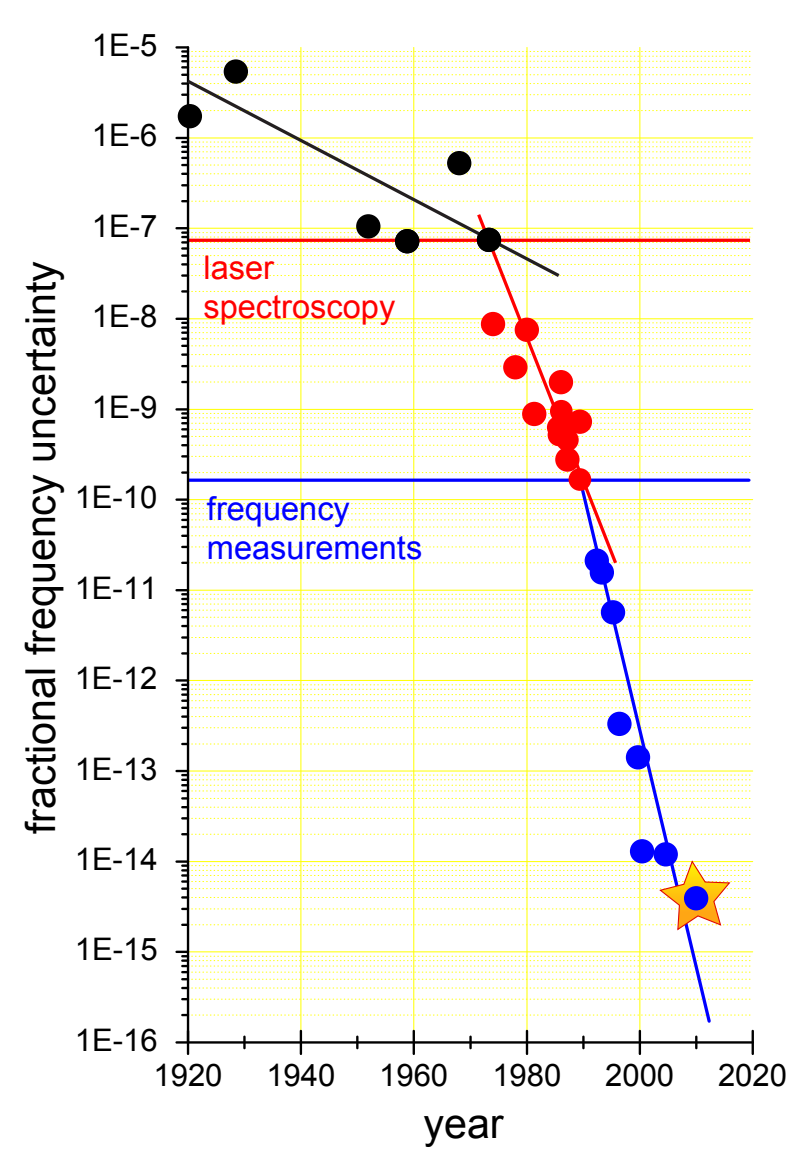

Figure 7.1: History of hydrogen spectroscopy. 


\section{Appendix A}

\section{Testing the Frequency Comb and Radio Frequency Equipment}

At the beginning of the work presented in this Thesis, the Erbium-doped fiber frequency comb and its radio frequency (rf) supply chain was only rudimentarily tested. The comb system is only specified to a fractional accuracy of $1 \times 10^{-14}$. Thus, the accuracy goal of $10^{-15}$ made a thorough testing necessary.

\section{A.1 Setup}

For the testing of our frequency comb system, Menlo Systems GmbH temporarily loaned us a second $250 \mathrm{MHz}$ repetition rate Erbium-doped fiber comb system. For the test, we referenced the two combs to the same rf reference oscillator and set up a beat with an ultra-stable laser near $1542 \mathrm{~nm}$. A sum of the beat notes with the proper sign must then be analyzed to hold the required precision (the mean has to yield the sum of the frequency combs' carrier envelope phases) and stability as most commonly characterized by an Allan deviation plot.

The setup is schematically depicted in Figure A.1. From the maser, the $10 \mathrm{MHz}$ signal is split by a distribution amplifier with $120 \mathrm{~dB}$ channel-to-channel isolation. Then, for each comb, the $10 \mathrm{MHz}$ signal is multiplied to $1 \mathrm{GHz}$ by three radio frequency multipliers. This signal is mixed with the fourth harmonic of the comb's repetition rate on a doublebalanced mixer used as phase detector for error signal creation. The repetition rate signal is amplified and filtered by a broad band pass filter (MiniCircuit VBFZ-925-S+).

\section{A.2 Cycle Slip Detection}

In previous measurements [13,3], beat note frequencies have been counted with HP/Agilent counters. Due to their random dead times, these counters need to be reset and externally triggered for each frequency point recorded with overlapping gates as required for cycle slip detection [56]. The reset leads to a significant delay so that for a $1 \mathrm{~s}$ gate the overall 


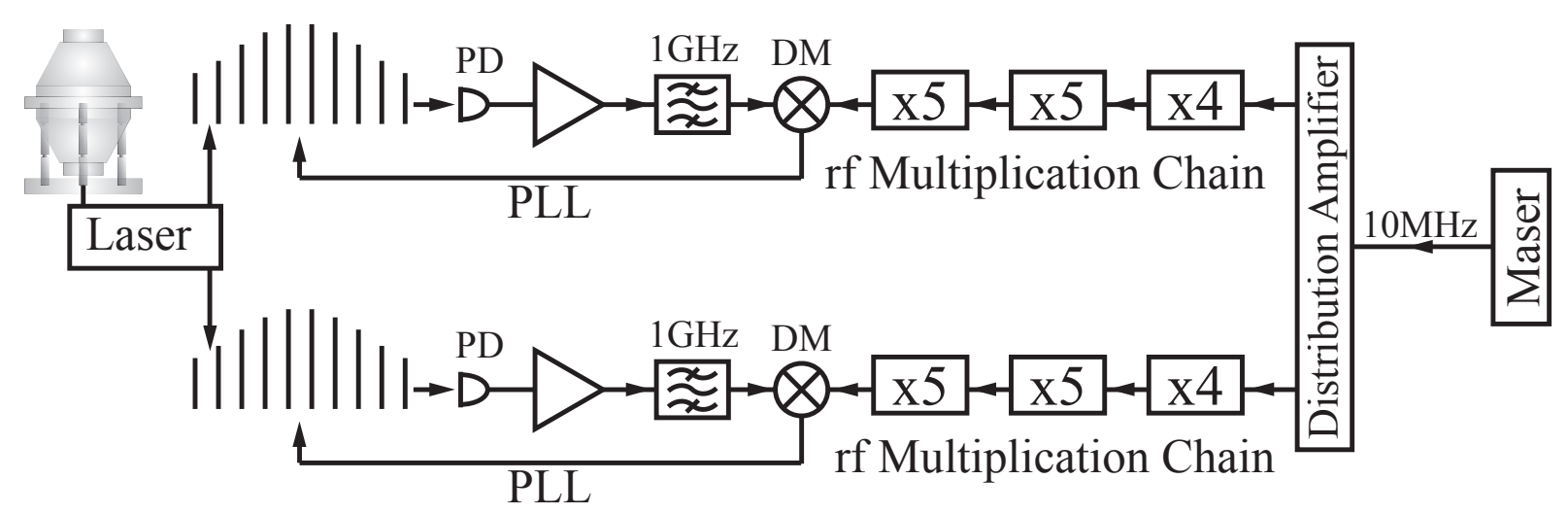

Figure A.1: Setup for the comparison of two frequency combs. PD - photo diode, PLL - phase lock loop, $1 \mathrm{GHz}-1 \mathrm{GHz}$ bandpass filter, DM - double-balanced mixer.

measurement time accumulates to roughly $3 \mathrm{~s}$. To speed up the frequency data acquisition time and significantly increase the amount of frequency data we introduce Klische \& Kramer counters, Model FX80, which allow a continuous, i.e. dead time free operation. For these new counters cycle slip criteria need to be defined.

The comparison of one laser frequency measured with two frequency combs allows to determine cycle slip criteria for exclusion of cycle slips which lead to a false frequency measurement. Here, a cycle slip denotes any error in frequency counting, which could be a lost cycle by the phase comparator of a frequency counter or of a phase lock loop [56].

Adding the laser beat notes with the two different combs with the right sign results in a constant function of time which is independent of the hydrogen maser noise as it is common mode to both signals. Cycle slips are immediately visible as outliers on this graph. The goal is to find objective criteria to identify these data points without having to operate two combs during all measurements. For this, two strategies are implemented. If the frequency is set by a phase lock loop to certain value, we count the actual frequency with a counter. Second, if the frequency is free running (like the laser-comb beat note), we count the signal with two counters after two filters with different bandwidths. Here, it is important to split the signal before digitizing it. In the setup described above, these criteria are:

- repetition rate: $\left|f_{\text {rep }}-f_{\text {rep }}^{\text {set }}\right| \leq 0.01 \mathrm{~Hz}$

- offset beat: $\left|f_{\mathrm{CEO}}-f_{\mathrm{CEO}}^{\text {set }}\right| \leq 0.5 \mathrm{~Hz}$

- laser-comb beat: $\left|f_{\text {beat }}^{1}-f_{\text {beat }}^{2}\right| \leq 0.1 \mathrm{~Hz}$

- fiber noise cancellation: $\left|f_{\text {fiber offset }}-f_{\text {fiber offset }}^{\text {set }}\right| \leq 0.01 \mathrm{~Hz}$ 


\section{A.3 Result}

During the investigation we find that the rf components are very sensitive to temperature fluctuations. For the rf multiplication chain components, we find that a temperature change $\Delta T / \Delta t=1 \mathrm{Ks}^{-1}$ cases a relative frequency drift of $\Delta f / f=5 \times 10^{-12}$. Keep in mind, that the components' frequency fluctuations are directly transferred to the optical by the frequency comb. To reach an accuracy level of $5 \times 10^{-16}$, the components need to be temperature stable within $1 \mathrm{mK}$ for $10 \mathrm{~s}$. This is now achieved by individually temperature stabilizing each component of the rf multiplication chain with Peltier elements. To avoid crosstalk between the temperature control loops we use two-point regulators providing either a high or low current to the Peltier elements. Although the temperature coefficients of the $1 \mathrm{GHz}$ bandpass filter and the double-balanced mixer are less severe, we still stabilize them individually with Peltier elements.

Also, it turns out that it is advisable to place the rf multiplication chain close to the maser and send the $1 \mathrm{GHz}$ signal through a temperature insensitive FSJ1 cable (Andrew Corp.) from the clock room to the hydrogen laboratory.

When time-averaging the beat note difference $\Delta f_{\text {combs }}$ for about a day, we find a mean value $\left\langle\Delta f_{\text {combs }}-\Delta f_{\mathrm{CEO}}\right\rangle_{1 \mathrm{~d}}=4.9(14) \mathrm{mHz}$ which is well compatible with zero* ${ }^{*}$ With the laser frequency of $f_{\text {Laser }}=1.94 \times 10^{14} \mathrm{~Hz}$ this translates to

$$
\frac{\left\langle\Delta f_{\text {combs }}-\Delta f_{\mathrm{CEO}}\right\rangle_{1 \mathrm{~d}}}{f_{\text {Laser }}}=2.5(7.2) \times 10^{-17}
$$

The Allan deviation flickers out at $3 \times 10^{-16}$ for averaging times of $700 \mathrm{~s}$ and above (compare Figure 3.6, pink trace).

${ }^{*}$ For comparison, before the individual stabilization (the whole $\mathrm{rf}$ multiplication chain was attached to an aluminum bread board temperature regulated by a single heating element), the mean was $\left\langle\Delta f_{\text {comb }}^{\text {poor }}{ }^{\mathrm{T}}\right.$ $\left.\Delta f_{\mathrm{CEO}}\right\rangle_{12 \mathrm{~h}} / f_{\text {Laser }}=-11.3(0.5) \times 10^{-16}$. This was a more than $20 \sigma$ deviation from zero. 
A.3. RESULT 


\section{Appendix B}

\section{Absolute Frequency of the Isotope Shift Hydrogen Data}

During the $2 S$ hyperfine splitting measurement [26] there was strong evidence that the excessive day-to-day scatter of the $1 S-2 S$ absolute frequency data taken in 1999 [13] and 2003 [3] was associated with the dye laser (also see Appendix D). Thus, having replaced the former spectroscopy laser with the all new diode laser based system (Section 3.2.2) there was good hope to have overcome the hidden systematic.

One of the motivations for the isotope shift measurement was to obtain enough hydrogen $1 S-2 S$ spectra to check whether the new system gave consistant statistics. Thus, we used the GPS-corrected hydrogen maser to generate an absolute frequency axis (with an uncertainty of a few parts in $10^{-14}$ ) for the hydrogen data taken during the isotope shift measurement (Chapter 4).

Figure B.1 shows the absolute frequency data for the $1 S-2 S$ transition in atomic hydrogen recorded during the isotope shift measurement. Each data point represents the average over one day of measurement, and has been linearly extrapolated to zero excitation power. At that time, there was no reasonable method to model the second order Doppler effect so it is not treated in the data presented. Thus, the frequency strongly depends on the delay. While the data presented in Figure B.1 does not provide good accuracy, it can be seen as a good measure of stability.

In contrast to the day-to-day scatter of the data taken in 1999 [13] and 2003 [3] which was on the order of $300 \mathrm{~Hz}$ and clearly larger than the individual error bars allow (see Figure 5.10), the data presented in Figure B.1 scatters more statistical on the order of $100 \mathrm{~Hz}$. With this encouraging result we decided to invite the French team with their mobile fountain clock to re-measure the $1 S-2 S$ absolute frequency as described in Chapter 5 . 


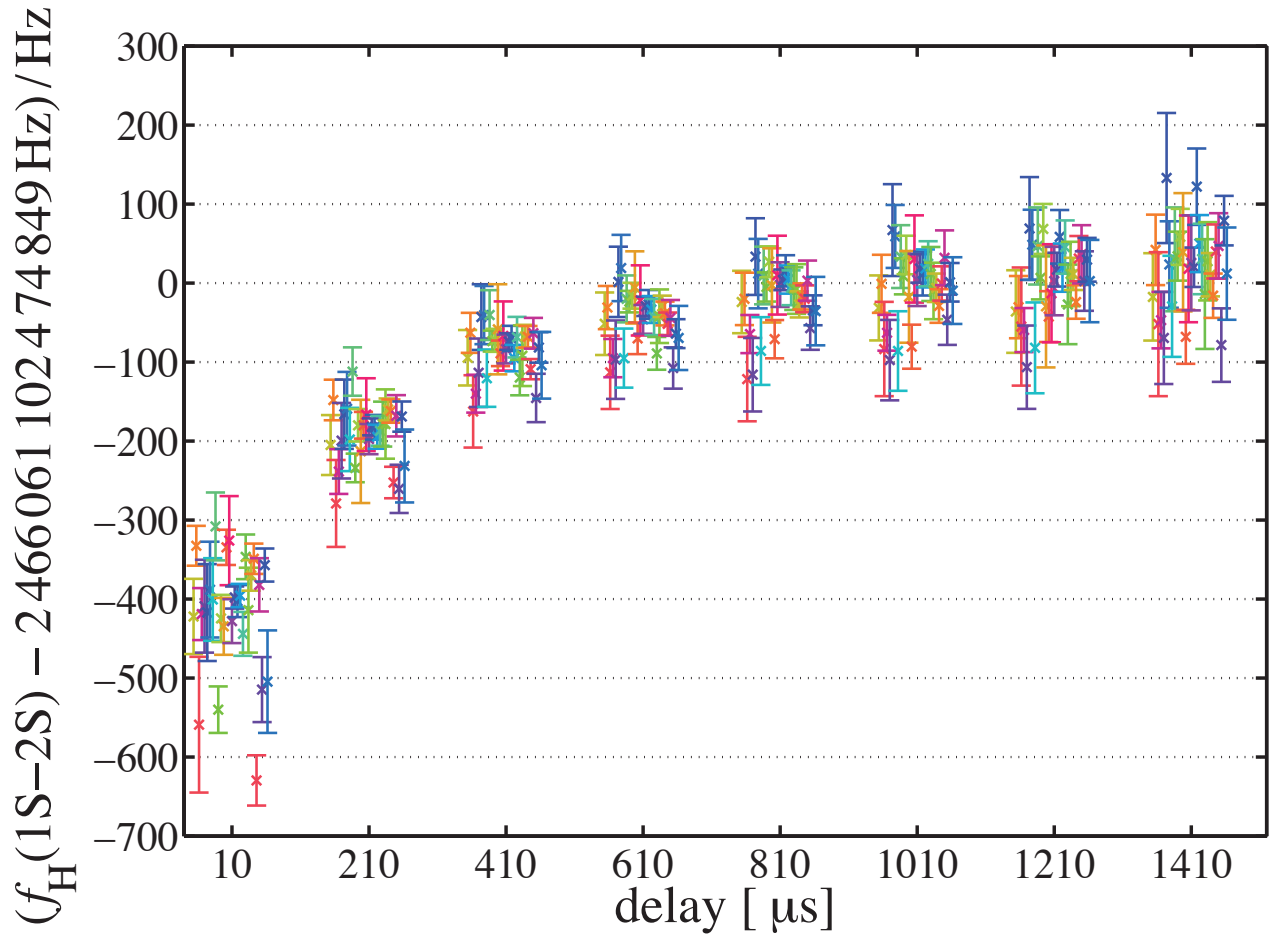

Figure B.1: Hydrogen absolute frequency data from the isotope shift measurement. Each data point represents the average over one day of measurement. It has been linearly extrapolated to zero intra-cavity power and the frequency is determined with the GPS-corrected hydrogen maser. One can clearly see the delay dependence of the $1 S-2 S$ frequency due to the second order Doppler effect which is not corrected for this graph, error bars are statistical only. 


\section{Appendix C}

\section{Analysis of Artificial Absolute Frequency Data}

\section{C.1 Test of the Line Shape Model}

The evaluation procedure used to analyze the data in Sections 5 and 6 shall be put under test in this Appendix. The geometry used in the simulation is shown in Figure C.4 at the end of this Appendix.

Currently, there is no simulation which can reproduce the line shape correctly at the desired level of accuracy, making a direct fit of the experimental line shape unfavorable. Since the simulation integrates the optical Bloch equations for a two-photon excitation using our experimental geometry and takes into account all additional effects we can think of - the second order Doppler effect, the ac Stark shift, ionization by a third photon the most likely explanation lies in a wrong assumption about the $1 S$ velocity distribution. However, a Maxwellian distribution poses a good approximation and can be modified to better represent the experimental conditions [79].

While a measurement of the $2 S$ velocity distribution is desirable to directly infer a second order Doppler correction, the $1 S$ distribution is needed as input parameter for the simulation. Since we have no means to detect ground state atomic hydrogen efficiently with good time resolution we attempted to reconstruct the $1 S$ distribution from the measured $2 S$ profiles using the MC simulation in a recursive way. However, this method becomes numerically unstable for small excitation probabilities for slow atoms. The method has not been proven to work properly so far.

An elegant way of circumventing this problem is to use the simulation only for verification of the overall algorithm applied to the experiment and - at most - use it to infer small corrections like the quadratic ac Stark shift.

Fig. C.1 shows a compilation of obtained $1 S-2 S$ frequencies for the applied analysis algorithm for varying simulation parameters. Here, $v^{2}$ and $v^{3}$ represent the velocity 


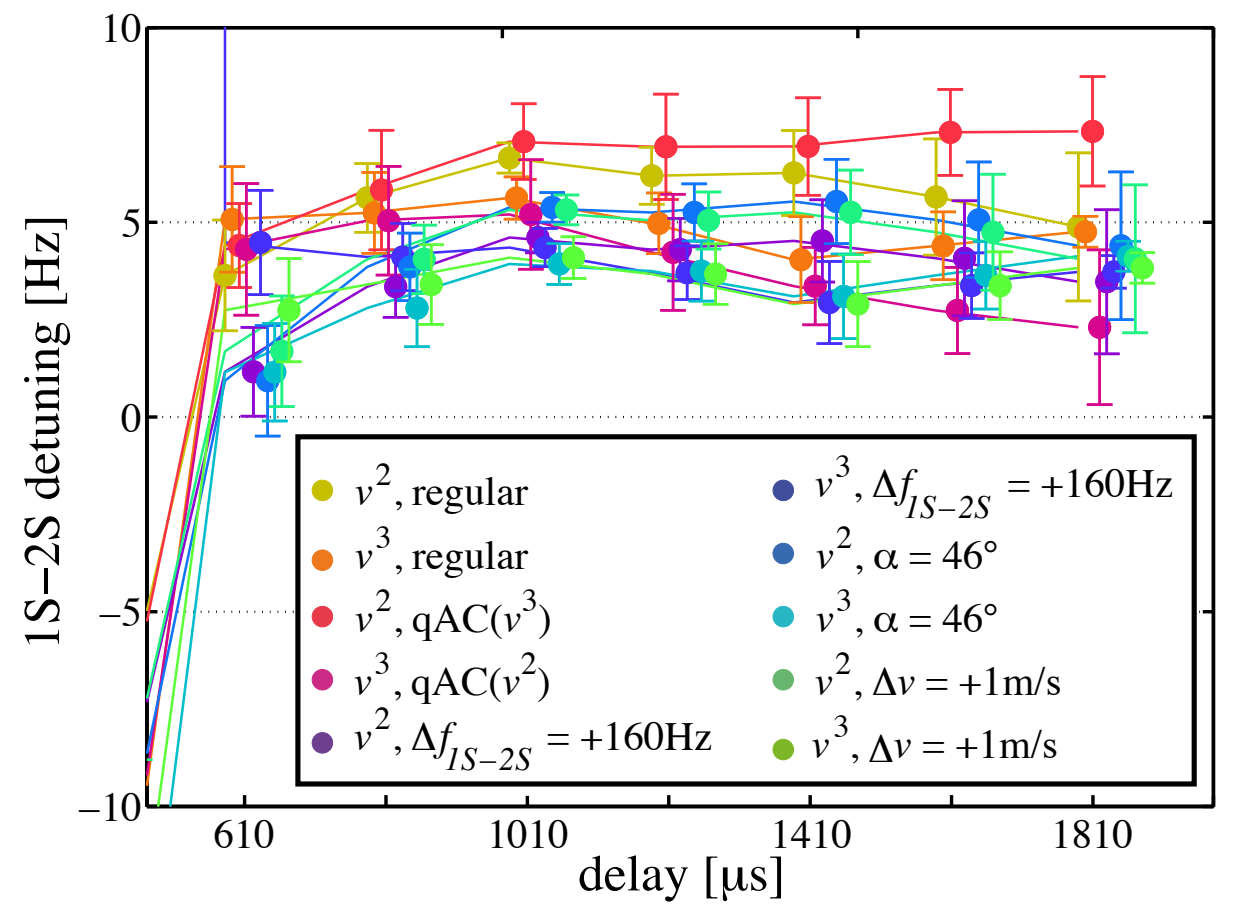

Figure C.1: Analysis of artificial data for various simulation parameters. See text for legend details.

dependence in the Maxwellian velocity distribution

$$
f(v)=N v^{n} \exp \left(\frac{-m v^{2}}{2 k T}\right)
$$

assumed for the $1 S$ atoms at the beginning of the excitation region. First, it should be mentioned that the evaluation algorithm leads to an agreement of the $1 S-2 S$ frequency for delays higher than $610 \mu$ s which obviously is a requirement for a working model. Also, for optimal conditions $\left(\mathrm{v}^{2}, \mathrm{v}^{3}\right.$ regular $)$ the algorithm produces a $5 \mathrm{~Hz}\left(2 \times 10^{-15}\right)$ offset which we regard as an overall uncertainty of the line shape model presented.

The various curves represent scenarios whose presence in the experiment cannot be ruled out by our uncertainty estimates. These are:

$\mathrm{qAC}\left(\mathrm{v}^{3}\right)$, $\mathrm{qAC}\left(\mathrm{v}^{3}\right)$ : Here, we use the quadratic ac Stark shift as determined for a Maxwellian distribution with $v^{3}$ to correct the numerical data calculated for $v^{2}$ and vice versa. It is an estimate for the deviation of the artificial $1 S$ velocity distribution from the experimental distribution.

$\Delta f_{1 S-2 S}= \pm 160 \mathrm{~Hz}$ : While measuring the velocity distribution with the Doppler laser via the $2 S-4 P$ transition the $243 \mathrm{~nm}$ spectroscopy laser was only kept on resonance within 
$\pm 160 \mathrm{~Hz}$.

$\alpha=46^{\circ}$ : The angle between the atomic hydrogen beam and the Doppler laser for the $2 S-4 P$ transition is $45(1)^{\circ}$.

$\Delta v= \pm 1 \mathrm{~m} / \mathrm{s}$ : Here, an offset of $1 \mathrm{~m} / \mathrm{s}$ is assumed in the $2 S$ velocity distribution. A possible origin would be a wrongly measured absolute frequency of the Doppler laser.

It is clear from Fig. C.1 that non of the described effects causes the model to break down.

\section{C.2 Comparison of Simulation and Experiment}

\section{C.2.1 Line width}

Figure C.2 shows the extrapolated line width at zero excitation power for two different Maxwell exponents along with the experimental data. Comparison with the value obtained

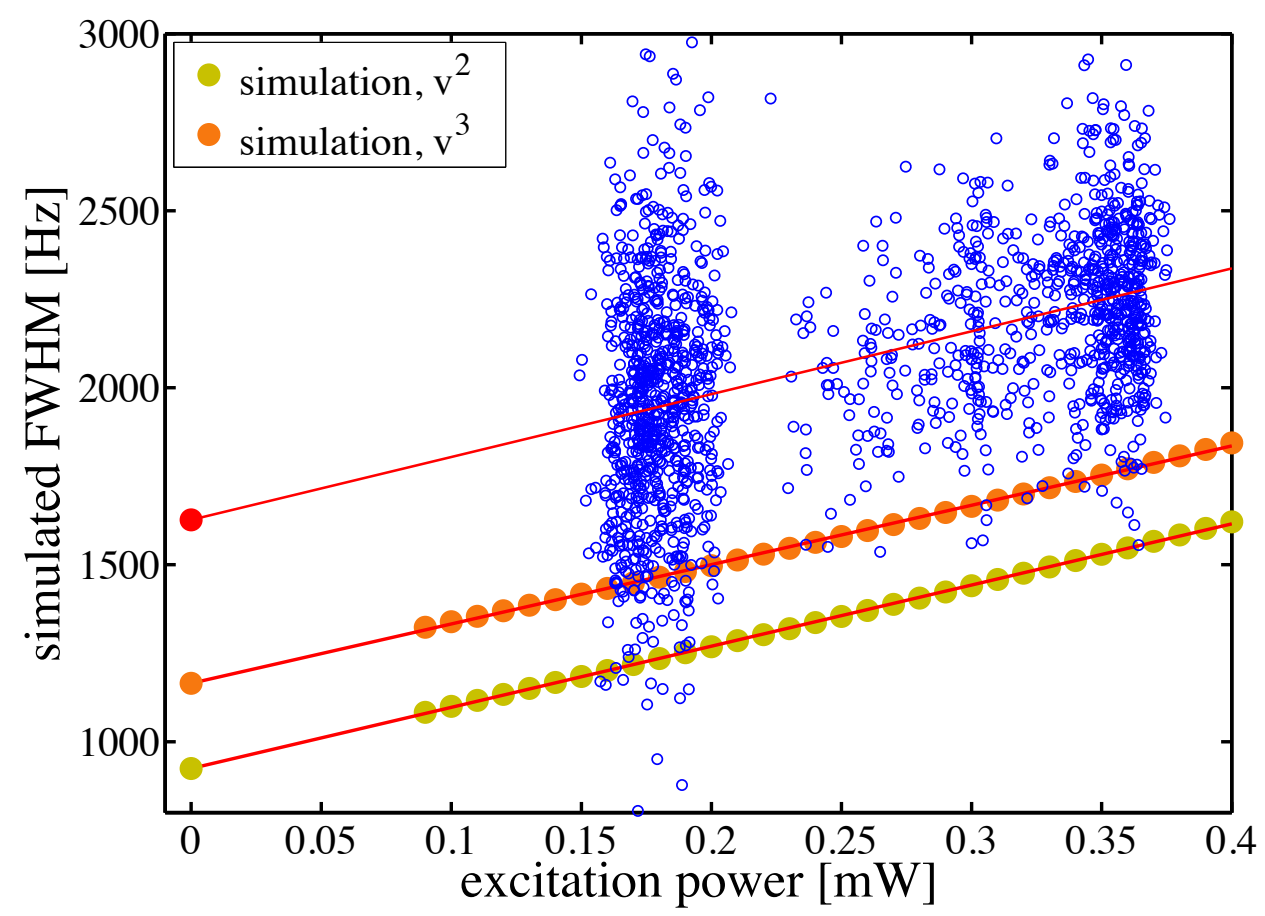

Figure C.2: Line width analysis of artificial data for two different Maxwell exponents simulated for delay $\tau_{6}=1010 \ldots 1210 \mu \mathrm{s}$. The simulated line width (yellow and orange points) systematically deviates from the experimentally observed line width (blue circles) of roughly $1.6 \mathrm{kHz}$ (also compare Figure 5.9). The red lines are obtained by linear regression of the respective data. 


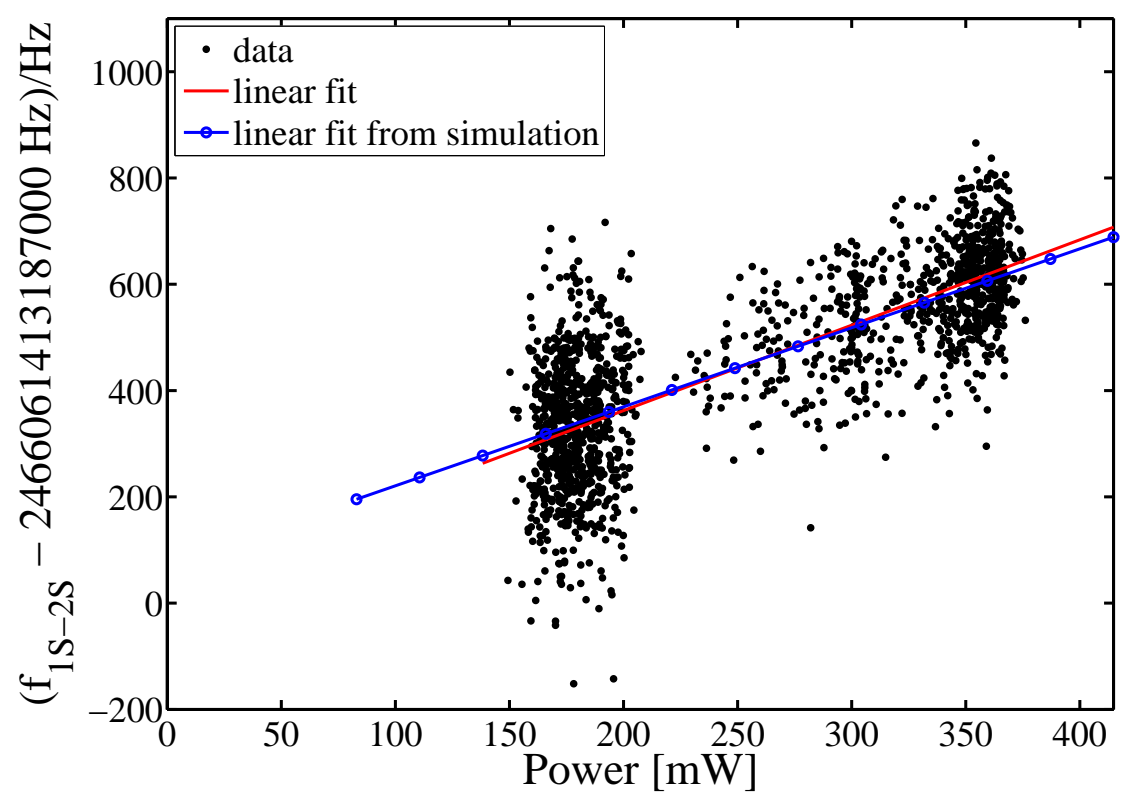

Figure C.3: Comparison of the linear ac Stark shift from experiment and simulation.

during the May 2010 measurement of roughly $1.6 \mathrm{kHz}$ (also see Figure 5.9) reveals a rather strong underestimation of the line width by the simulation. This deviation is not yet understood and might be part of the problem that to date a direct fit of the experimental data with the simulated spectra is not satisfactory at the required uncertainy level of $10^{-15}$. However, the influence of the incorrect line width on the conclusions drawn from the simulation analysis has been estimated to be negligible by folding the simulated line shape with a Lorentzian. Picking the broadening such that the experimental line width is achieved does not change the conclusions drawn from the simulation.

\section{C.2.2 ac Stark shift}

To exclude the most obvious candidate, which is power broadening, as an explanation for the discrepancy of experimentally observed and simulated line width, we compare the linear ac Stark shift as obtained from experiment and simulation. Figure C.3 shows that the ac Stark shifts agree well. 


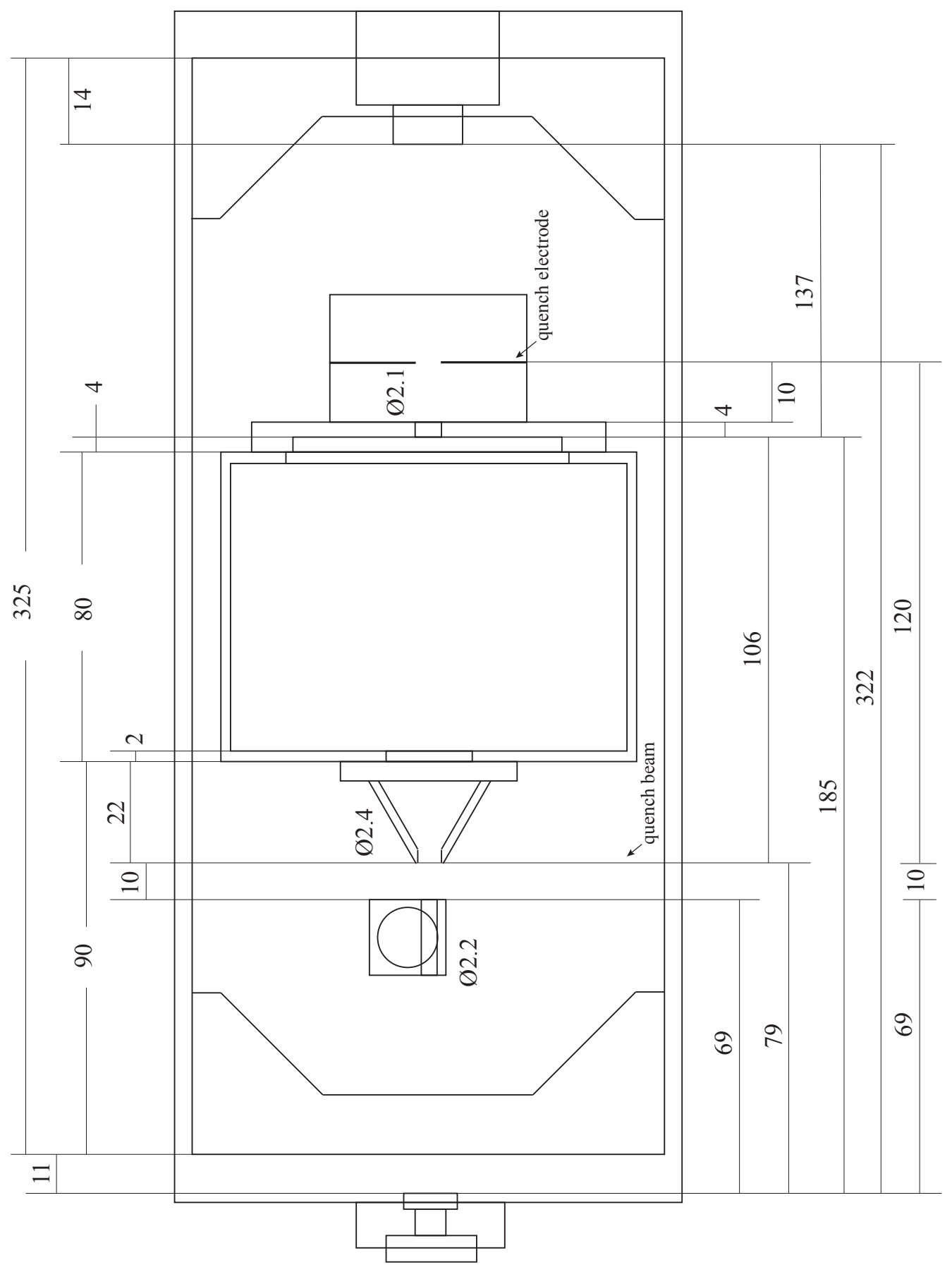

Figure C.4: Beam apparatus geometry used for simulation. All dimensions are in millimeters. 


\section{Appendix D}

\section{Dye laser lock}

During the HFS measurement in 2008 [26], the $1 S-2 S$ transition was excited with the dye laser. The extended cavity diode laser locked to a newly built ULE cavity stabilized at its critical temperate [35] was used as clock laser. The beat note of both lasers revealed that the dye laser's frequency is modulated $(1 \mathrm{kHz}$ peak-to-peak at $486 \mathrm{~nm}$ with a periodicity of $1000 \mathrm{~s}$, see Fig. D.2). Also, re-locking the laser resulted in jumps. This inaccurate lock is blamed for the formerly uncharacterized day-to-day scatter.

The dye laser is locked to two different cavities simultaneously (Fig. D.1):

1. side of fringe lock to Coherent cavity (compare Fig. D.3)

2. PDH lock to ULE cavity (Figure 3.5, FP3) built by Marc Fischer.

The lock is established by the Coherent locking electronics. The error signals of both

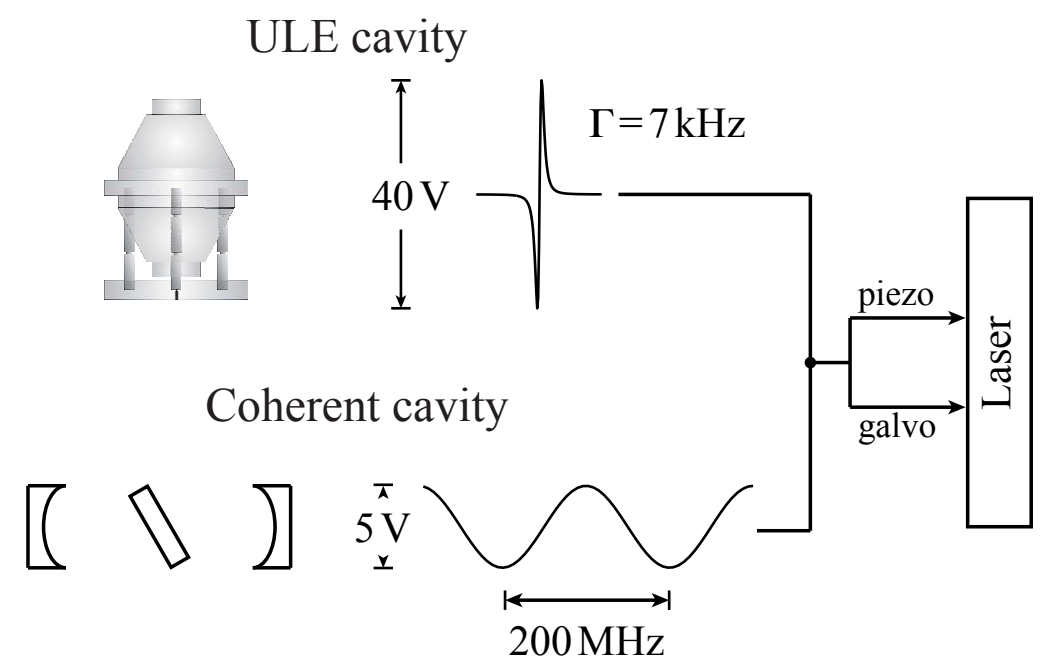

Figure D.1: Locking schematic of the dye laser. The error signals of both cavities are simply combined at a BNC T-junction effectively shortening the outputs of two amplifiers. 
cavities are simply overlaid at a BNC T-junction effectively shortening the outputs of two amplifiers. The peak-to-peak voltage of the Coherent signal is $5 \mathrm{~V}$, for the ULE cavity it was $40 \mathrm{~V}$ during the 2003 measurement. The error signals are sketched in Fig. D.1.

Assuming similar output resistances of the amplifiers the frequency sensitivity of the combined error signal can be described as

$$
\begin{aligned}
S_{\text {err }} & =\frac{40 \mathrm{~V}}{7 \mathrm{kHz}} V_{\mathrm{ULE}}+\frac{5 \mathrm{~V}}{100 \mathrm{MHz}} V_{\text {coherent }} \\
& =5.7 \times 10^{-3} \frac{\mathrm{V}}{\mathrm{Hz}} \cdot V_{\mathrm{ULE}}+5 \times 10^{-8} \frac{\mathrm{V}}{\mathrm{Hz}} \cdot V_{\text {coherent }}
\end{aligned}
$$

with the Coherent cavity fringe spacing of $100 \mathrm{MHz}$ and a ULE cavity line width of $7 \mathrm{kHz}$. We see that the frequency sensitivity to the Coherent cavity is suppressed by $10^{-5}$.

This explains why - although the Coherent cavity's drift is believed to exceed $10 \mathrm{kHz} / \mathrm{s}$ - the drift of the combined system is at the $\mathrm{Hz} / \mathrm{s}$ level. Looking at Fig. D.2 it seems that the laser frequency is modulated with a period of $\sim 1000 \mathrm{~s}$ and a modulation depth of $1000 \mathrm{~Hz}$. The modulation time scale suggests the Coherent cavity's temperature stabilization as the cause of the modulation. If the laser stays in lock during the comb averaging time the modulation might be reproduced correctly.

However, if the laser looses it's lock to the ULE cavity there will be problems: The usual unlocking scenario is this: A bubble* within the dye jet causes a mode jump of the dye laser. Thus, the lock to the ULE cavity is lost. The Coherent cavity is designed with a very wide capture range so that its lock is kept. To match the laser to the ULE cavity one has to turn the intra Coherent cavity galvo (Fig. D.3) therefore changing the frequency of the Coherent cavity. Thus, when the lock to the ULE cavity is re-established the laser frequency can differ by the modulation amplitude $(1 \mathrm{kHz})$ from the frequency prior to unlock.

In conclusion it has to be said that the dye laser lock is not well suited for a measurement on the $10^{-14}$ level. Fortunately, the 2010 measurement confirms that the modulation averages out if enough hydrogen lines are recorded.

\footnotetext{
*For the operation of the dye laser at $486 \mathrm{~nm}$, the dye jet needs to be run at a pressure of $10.5 \mathrm{bar}$. The formation of bubbles is almost inevitable at such a high pressure.
} 


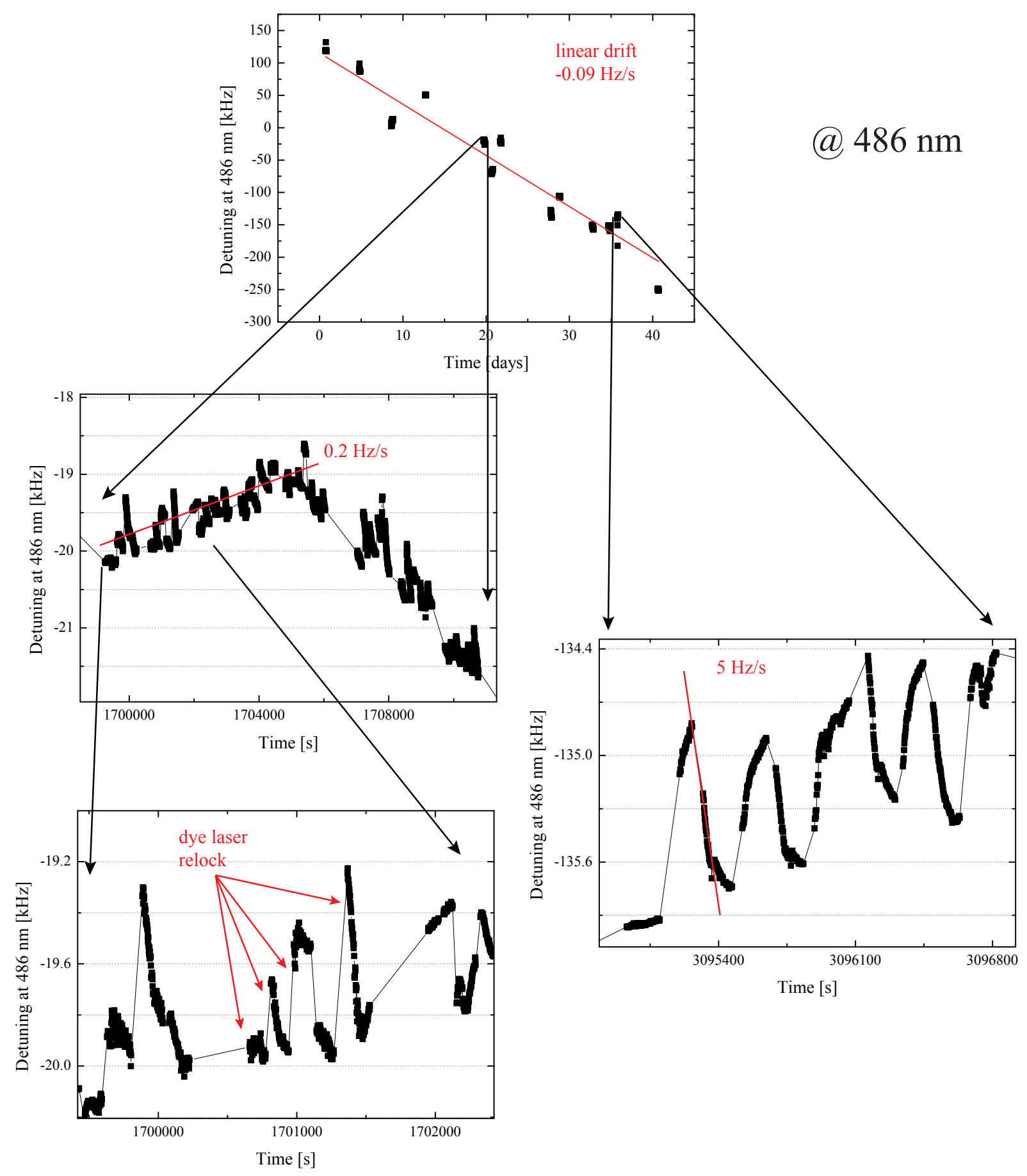

Figure D.2: Relative drift of dye laser and ECDL1 (The drift of ECDL1 $(100 \mathrm{mHz} / \mathrm{s}$ at $486 \mathrm{~nm})$ can be neglected compared to the dye laser drift.) The time scale zooms in from top to bottom. 

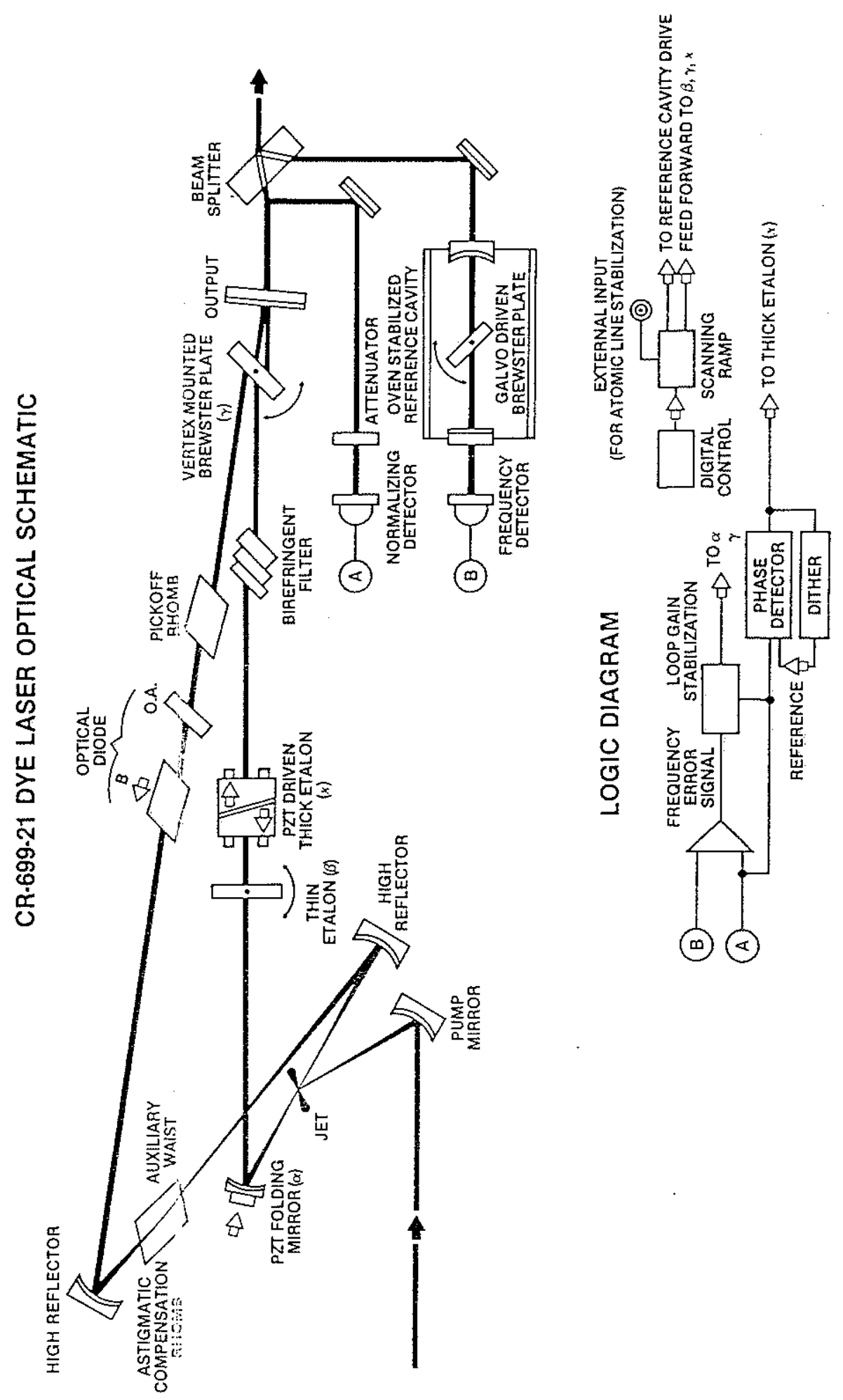

Figure D.3: Dye laser schematic [93]. 


\section{Appendix E}

\section{Collection of Experimental Parameters and Procedures}

This Appendix is intended as a quick-reference for future students at the experiments. For the sake of shortness and ease, the occasional use of lab slang is intended. It should be understood as an alphabetically ordered summary of my lab book.

\section{cryo-pump and freezing cycle}

- The cryo-pump's displacer was changed in October 2010 by a Leybold technician. After a few days it turned very noisy (banging noise) and needed to be exchanged again. There has been a bad charge in Leybold production.

- In the beginning of a measurement, we measure 80 points for one line and pump for the equivalent of 40 . In the end, after 80 points of measurement, pumping on the level 60 to 80 points is necessary.

- The nozzle typically freezes within 45 min, leading to 15 lines per cycle in the beginning and 10 lines per cycle at the end of a measurement day.

\section{data evaluation}

- Individual lines are fitted with independent Lorentzians for each delay.

- Line centers (without assigned uncertainties) are extrapolated to zero excitation power for each freezing cycle. The uncertainty for this extrapolation value is extracted from the linear regression.

- The maser frequency is corrected for each freezing cycle when using a Cs fountain clock and for each day if using GPS. 


\section{discharge}

- $30 \mathrm{~W}$ microwave power

- Sapphire tube: $d_{\text {out }}=9.2 \mathrm{~mm}$, innter diameter $d_{\text {in }}=7.6 \mathrm{~mm}$, some tubes give nice discharge, with others the count rate is extremely low

- cooling: cold nitrogen gas, $T=-25^{\circ} \mathrm{C}$ when leaving microwave cavity

- Teflon capillary

- hydrogen/deuterium pressure: 1 mbar

- A leakage of $1 \times 10^{-3}$ mbar of air into the discharge region improves count rate but accelerates mirror degradation.

- A bright pink discharge color is a good indicator of proper operation.

\section{ECDL1 power levels}

- seed power: $40 \mathrm{~mW}$

- For the absolute frequency measurement in May, the power levels were:

- $972 \mathrm{~nm}: 670 \mathrm{~mW}$

- $486 \mathrm{~nm}: 280 \mathrm{~mW}$

$-243 \mathrm{~nm}: 15 \mathrm{~mW}$

- For the absolute frequency measurement in November, the power levels were:

- $972 \mathrm{~nm}: 650 \mathrm{~mW}$

- $486 \mathrm{~nm}: 250 \mathrm{~mW}$

- $243 \mathrm{~nm}: 12 \mathrm{~mW}$

\section{ECDL1 wave lengths}

When measured with the WS7 wavemeter, the following wavelengths are measured for the right Fabry Perot cavity modes (the value in curly brackets may deviate due to the temperature dependents of the wavemeter)

- $\mathrm{H}: \lambda_{\mathrm{H}}=972.538\{36\} \mathrm{nm}, \mathrm{T}=14.98^{\circ} \mathrm{C}$

- D: $\lambda_{\mathrm{D}}=972.273\{63\} \mathrm{nm}, \mathrm{T}=15.90^{\circ} \mathrm{C}$ 


\section{enhancement cavity}

- incoupler: flat, $T=0.98, \mathrm{REO} \mathrm{PO} \# \mathrm{~S} 62368 / 410$

- outcoupler: $r=4 \mathrm{~m}, T=1.3 \times 10^{-4}$, REO PO\# 468093/10410K

- mirror separation: $L=32.2 \mathrm{~cm}$

- mirrors are cleaned with methanol by four to five wipes

- maximum power per direction: $\sim 380 \mathrm{~mW}$ with $15 \mathrm{~mW}$ of $243 \mathrm{~nm}$ radiation

\section{liquid helium transfer tube}

- There are two proven ways to change the liquid helium dewar without cloaking the helium transfer tube:

- take out helium transfer tube, flush with gaseous helium for at least two days

- transfer helium transfer tube from one dewar to the other instantaneously without exposing it to air for more than a few seconds

\section{nozzle}

- cleaning: mechanically polish with grinding paste and thread, optional sonication in acetone, then $10 \mathrm{~min}$ sonication in soap water, $10 \mathrm{~min}$ sonication in tapped water, let air dry or use heat gun if in hurry

- optimal temperaturs: hydrogen: $T=5.8 \mathrm{~K}, T=7.5 \mathrm{~K}$, measured $1 \mathrm{~cm}$ above the nozzle orifice directly on the copper block

\section{quench field}

- One electrode is just a silver coated wire attached to the chamber for grounding. The second electrode is a homemade coax-cable: A Kapton coated wire is inserted in a stainless steel tube. The coating is not stripped at all, only the front face of the wire is de-insulated. The wire sticks out of the tube by $0.1 \mathrm{~mm}$ and is put to $+30 \mathrm{~V}$.

\section{venting}

- We use nitrogen evaporated from liquid nitrogen to avoid contamination with oil (like present in nitrogen from bottles). 


\section{Bibliography}

[1] Huber, A., Udem, Th., Gross, B., Reichert, J., Kourogi, M., Pachucki, K., Weitz, M., and Hänsch, T. W., "Hydrogen-deuterium $1 S-2 S$ isotope shift and the structure of the deuteron," Phys. Rev. Lett. 80, 468-471 (Jan 1998).

[2] Jentschura, U., Matveev, A., Parthey, C., Alnis, J., Pohl, R., Udem, Th., Kolachevsky, N., and Hänsch, T., "Hydrogen-deuterium isotope shift: From the $1 S-2 S$-transition frequency to the proton-deuteron charge-radius difference," Phys. Rev. A 83, 042505 (Apr 2011).

[3] Fischer, M., Kolachevsky, N., Zimmermann, M., Holzwarth, R., Udem, Th., Hänsch, T. W., Abgrall, M., Grünert, J., Maksimovic, I., Bize, S., Marion, H., Santos, F. P. D., Lemonde, P., Santarelli, G., Laurent, P., Clairon, A., Salomon, C., Haas, M., Jentschura, U. D., and Keitel, C. H., "New limits on the drift of fundamental constants from laboratory measurements," Phys. Rev. Lett. 92, 230802 (Jun 2004).

[4] Fortier, T. M., Ashby, N., Bergquist, J. C., Delaney, M. J., Diddams, S. A., Heavner, T. P., Hollberg, L., Itano, W. M., Jefferts, S. R., Kim, K., Levi, F., Lorini, L., Oskay, W. H., Parker, T. E., Shirley, J., and Stalnaker, J. E., "Precision atomic spectroscopy for improved limits on variation of the fine structure constant and local position invariance," Phys. Rev. Lett. 98, 070801 (Feb 2007).

[5] Hänsch, T. W., "Nobel lecture: Passion for precision," (2005).

[6] Gabrielse, G., Hanneke, D., Kinoshita, T., Nio, M., and Odom, B., "New determination of the fine structure constant from the electron $g$ value and qed," Phys. Rev. Lett. 97, 030802 (Jul 2006).

[7] Hanneke, D., Fogwell, S., and Gabrielse, G., "New measurement of the electron magnetic moment and the fine structure constant," Phys. Rev. Lett. 100, 120801 (Mar 2008).

[8] Mohr, P. J., Taylor, B. N., and Newell, D. B., "CODATA recommended values of the fundamental physical constants: 2006," Rev. Mod. Phys. 80, 633-730 (Jun 2008).

[9] Bernauer, J. C., Achenbach, P., Ayerbe Gayoso, C., Böhm, R., Bosnar, D., Debenjak, L., Distler, M. O., Doria, L., Esser, A., Fonvieille, H., Friedrich, J. M., Friedrich, J., 
Gómez Rodríguez de la Paz, M., Makek, M., Merkel, H., Middleton, D. G., Müller, U., Nungesser, L., Pochodzalla, J., Potokar, M., Sánchez Majos, S., Schlimme, B. S., Sirca, S., Walcher, T., and Weinriefer, M., "High-precision determination of the electric and magnetic form factors of the proton," Phys. Rev. Lett. 105, 242001 (Dec 2010).

[10] Zhan, X., Allada, K., Armstrong, D., Arrington, J., Bertozzi, W., Boeglin, W., Chen, J.-P., Chirapatpimol, K., Choi, S., Chudakov, E., Cisbani, E., Decowski, P., Dutta, C., Frullani, S., Fuchey, E., Garibaldi, F., Gilad, S., Gilman, R., Glister, J., Hafidi, K., Hahn, B., Hansen, J.-O., Higinbotham, D., Holmstrom, T., Holt, R., Huang, J., Huber, G., Itard, F., de Jager, C., Jiang, X., Johnson, M., Katich, J., de Leo, R., LeRose, J., Lindgren, R., Long, E., Margaziotis, D., Beck, S. M.-T., Meekins, D., Michaels, R., Moffit, B., Norum, B., Olson, M., Piasetzky, E., Pomerantz, I., Protopopescu, D., Qian, X., Qiang, Y., Rakhman, A., Ransome, R., Reimer, P., Reinhold, J., Riordan, S., Ron, G., Saha, A., Sarty, A., Sawatzky, B., Schulte, E., Shabestari, M., Shahinyan, A., Shneor, R., Sirca, S., Solvignon, P., Sparveris, N., Strauch, S., Subedi, R., Sulkosky, V., Vilardi, I., Wang, Y., Wojtsekhowski, B., Ye, Z., and Zhang, Y., "High-precision measurement of the proton elastic form factor ratio $\mu p G E / G M$ at low $Q^{2}, "$ Physics Letters B 705(1-2), 59 - 64 (2011).

[11] Pohl, R., Antognini, A., Nez, F., Amaro, F. D., Biraben, F., Cardoso, J. M. R., Covita, D. S., Dax, A., Dhawan, S., Fernandes, L. M. P., Giesen, A., Graf, T., Hänsch, T. W., Indelicato, P., Julien, L., Kao, C.-Y., Knowles, P., Lopes, J. A. M., Le Bigot, E.-O., Liu, Y.-W., Ludhova, L., Monteiro, C. M. B., Mulhauser, F., Nebel, T., Rabinowitz, P., dos Santos, J. M. F., Schaller, L. A., Schuhmann, K., Schwob, C., Taqqu, D., Veloso, J. F. C. A., and Kottmann, F., "The size of the proton," Nature 466, 213-216 (2010).

[12] Nebel, T., "The lamb shift in muonic hydorgen," PhD Thesis, MPQ report 332 (2010).

[13] Niering, M., Holzwarth, R., Reichert, J., Pokasov, P., Udem, Th., Weitz, M., Hänsch, T. W., Lemonde, P., Santarelli, G., Abgrall, M., Laurent, P., Salomon, C., and Clairon, A., "Measurement of the hydrogen $1 S-2 S$ transition frequency by phase coherent comparison with a microwave cesium fountain clock," Phys. Rev. Lett. 84, 5496-5499 (Jun 2000).

[14] Rosenband, T., Hume, D. B., Schmidt, P. O., Chou, C. W., Brusch, A., Lorini, L., Oskay, W. H., Drullinger, R. E., Fortier, T. M., Stalnaker, J. E., Diddams, S. A., Swann, W. C., Newbury, N. R., Itano, W. M., Wineland, D. J., and Bergquist, J. C., "Frequency ratio of $\mathrm{Al}+$ and $\mathrm{Hg}+$ single-ion optical clocks; metrology at the 17th decimal place," Science 319(5871), 1808-1812 (2008).

[15] Colladay, D. and Kostelecký, V. A., "Lorentz-violating extension of the standard model," Phys. Rev. D 58, 116002 (Oct 1998). 
[16] Andresen, G. B., Ashkezari, M. D., Baquero-Ruiz, M., Bertsche, W., Bowe, P. D., Butler, E., Cesar, C. L., Chapman, S., Charlton, M., Deller, A., Eriksson, S., Fajans, J., Friesen, T., Fujiwara, M. C., Gill, D. R., Gutierrez, A., Hangst, J. S., Hardy, W. N., Hayden, M. E., Humphries, A. J., Hydomako, R., Jenkins, M. J., Jonsell, S., Jrgensen, L. V., Kurchaninov, L., Madsen, N., Menary, S., Nolan, P., Olchanski, K., Olin, A., Povilus, A., Pusa, P., Robicheaux, F., Sarid, E., Nasr, S. S. E., Silveira, D. M., So, C., Storey, J. W., Thompson, R. I., Van Der Werf, D. P., Wurtele, J. S., and Yamazaki, Y., "Confinement of antihydrogen for 1,000 seconds," Nat Phys 7, 558-564 (07 2011).

[17] Andresen, G. B., Ashkezari, M. D., Baquero-Ruiz, M., Bertsche, W., Bowe, P. D., Butler, E., Cesar, C. L., Chapman, S., Charlton, M., Deller, A., Eriksson, S., Fajans, J., Friesen, T., Fujiwara, M. C., Gill, D. R., Gutierrez, A., Hangst, J. S., Hardy, W. N., Hayden, M. E., Humphries, A. J., Hydomako, R., Jenkins, M. J., Jonsell, S., Jrgensen, L. V., Kurchaninov, L., Madsen, N., Menary, S., Nolan, P., Olchanski, K., Olin, A., Povilus, A., Pusa, P., Robicheaux, F., Sarid, E., Nasr, S. S. E., Silveira, D. M., So, C., Storey, J. W., Thompson, R. I., Van Der Werf, D. P., Wurtele, J. S., and Yamazaki, Y., "Trapped antihydrogen," Nature 468(7324), 673-676 (2010).

[18] Amoretti, M., Amsler, C., Bonomi, G., Bouchta, A., Bowe, P., Carraro, C., Cesar, C. L., Chaliton, M., Collier, M. J. T., Doser, M., Filippini, V., Fine, K. S., Fontana, A., Fujiwara, M. C., Funakoshi, R., Genova, P., Hangst, J. S., Hayano, R. S., Holzscheiter, M. H., Jrgensen, L. V., Lagomarsino, V., Landua, R., Lindelf, D., Lodi Rizzini, E., Macri, M., Madsen, N., Manuzio, G., Marchesotti, M., Montagna, P., Pruys, H., Regenfus, C., Riedler, P., Rochet, J., Rotondi, A., Rouleau, G., Testera, G., Variola, A., Watson, T. L., and Van der Werf, D. P., "Production and detection of cold antihydrogen atoms," Nature 419(6906), 456-459 (2002).

[19] Gabrielse, G., Bowden, N. S., Oxley, P., Speck, A., Storry, C. H., Tan, J. N., Wessels, M., Grzonka, D., Oelert, W., Schepers, G., Sefzick, T., Walz, J., Pittner, H., Hänsch, T. W., and Hessels, E. A., "Background-free observation of cold antihydrogen with field-ionization analysis of its states," Phys. Rev. Lett. 89, 213401 (Oct 2002).

[20] Sapirstein, J. and Yennie, D. R., [Quantum electrodynamics], 560-672, Vol. 7 of Advanced Series on Directions in High Energy Physics, edited by T. Kinoshita, World Scientific (1990).

[21] Bethe, H. and Salpeter, E., [Quantum mechanics of one-and two-electron atoms], Plenum Pub. Corp. (1977).

[22] Eides, M. I., Grotch, H., and Shelyuto, V. A., "Theory of light hydrogenlike atoms," Physics Reports 342(2-3), 63 - 261 (2001).

[23] Karshenboim, S. G., "Precision physics of simple atoms: QED tests, nuclear structure and fundamental constants," Physics Reports 422(1-2), 1 - 63 (2005). 
[24] Zwanziger, D. E., " $\alpha^{3}$ corrections to hyperfine structure in hydrogenic atoms," Phys. Rev. 121, 1128-1142 (Feb 1961).

[25] Sternheim, M. M., "State-dependent mass corrections to hyperfine structure in hydrogenic atoms," Phys. Rev. 130, 211-222 (Apr 1963).

[26] Kolachevsky, N., Matveev, A., Alnis, J., Parthey, C. G., Karshenboim, S. G., and Hänsch, T. W., "Measurement of the $2 S$ hyperfine interval in atomic hydrogen," Phys. Rev. Lett. 102, 213002 (May 2009).

[27] Essen, L., Donaldson, R. W., Bangham, M. J., and Hope, E. G., "Frequency of the hydrogen maser," Nature 229(5280), 110-111 (1971).

[28] Wineland, D. J. and Ramsey, N. F., "Atomic deuterium maser," Phys. Rev. A 5, 821-837 (Feb 1972).

[29] Kolachevsky, N., Fendel, P., Karshenboim, S. G., and Hänsch, T. W., "2S hyperfine structure of atomic deuterium," Phys. Rev. A 70, 062503 (Dec 2004).

[30] Kolachevsky, N., "PhD thesis,".

[31] Huber, A., Gross, B., Weitz, M., and Hänsch, T. W., "High-resolution spectroscopy of the $1 S-2 S$ transition in atomic hydrogen," Phys. Rev. A 59, 1844-1851 (Mar 1999).

[32] Parthey, C. G., Matveev, A., Alnis, J., Bernhard, B., Beyer, A., Holzwarth, R., Maistrou, A., Pohl, R., Predehl, K., Udem, Th., Wilken, T., Kolachevsky, N., Hänsch, T. W., Abgrall, M., Rovera, D., Salomon, C., and Laurent, P., "Improved measurement of the hydrogen $1 S-2 S$ transition frequency," accepted for publication by Phys. Rev. Lett., arXiv:1107.3101v1 (2011).

[33] Fischer, M. C., "Höchstauflösende laserspektroskopie an atomarem wasserstoff," (2004).

[34] Kolachevsky, N., Alnis, J., Parthey, C. G., Matveev, A., Landig, R., and Hänsch, T. W., "Low phase noise diode laser oscillator for $1 S-2 S$ spectroscopy in atomic hydrogen," accepted for publication by Opt. Lett.; arXiv:1107.1649v1 (2011).

[35] Alnis, J., Matveev, A., Kolachevsky, N., Udem, Th., and Hänsch, T. W., "Subhertz linewidth diode lasers by stabilization to vibrationally and thermally compensated ultralow-expansion glass Fabry-Pérot cavities," Phys. Rev. A 77, 053809 (May 2008).

[36] Notcutt, M., Ma, L.-S., Ye, J., and Hall, J. L., "Simple and compact 1-Hz laser system via an improved mounting configuration of a reference cavity," Opt. Lett. 30, 1815-1817 (Jul 2005). 
[37] Barnes, J. A., Chi, A. R., Cutler, L. S., Healey, D. J., Leeson, D. B., McGuniga, T. E., Mullen, J. A., Smith, W. L., Sydnor, R. L., Vessot, R. F. C., and Winkler, G. M. R., "Characterization of frequency stability," IEEE Transactions on Instrumentation and Measurement 20(2), 105 (1971).

[38] Bize, S., Laurent, P., Abgrall, M., Marion, H., Maksimovic, I., Cacciapuoti, L., Grnert, J., Vian, C., dos Santos, F. P., Rosenbusch, P., Lemonde, P., Santarelli, G., Wolf, P., Clairon, A., Luiten, A., Tobar, M., and Salomon, C., "Advances in atomic fountains," Comptes Rendus Physique 5(8), 829 - 843 (2004).

[39] Rovera, D., "Frequency comparison (H_MASER $140 \quad$ 0890) (LNE-SYRTE-FOM) for the period MJD 55344 to MJD 55359," ftp://ftp2.bipm.org/pub/tai/data/PFS_reports/syrte-fom_55344-55359.pdf.

[40] Weyers, S., Hübner, U., Schröder, R., Tamm, C., and Bauch, A., "Uncertainty evaluation of the atomic caesium fountain CSF1 of the PTB," Metrologia 38(4), 343 (2001).

[41] Weyers, S., Bauch, A., Schröder, R., and Tamm, C., [Proceedings of the 6th Symposium on Frequency Standards and Metrology], 64-71, World Scientific (2001). The atomic caesium fountain CSF1 of PTB.

[42] Predehl, K., Grosche, G., Raupach, S. M. F., Droste, S., Alnis, J., Legero, T., Hänsch, T. W., Udem, Th., Holzwarth, R., and Schnatz, H., "A continental-scale optical fiber link for frequency metrology at the 19th decimal place," in preparation.

[43] Terra, O., Grosche, G., Predehl, K., Holzwarth, R., Legero, T., Sterr, U., Lipphardt, B., and Schnatz, H., "Phase-coherent comparison of two optical frequency standards over $146 \mathrm{~km}$ using a telecommunication fiber link," Applied Physics B: Lasers and Optics 97, 541-551 (2009). 10.1007/s00340-009-3653-2.

[44] Kluge, H.-J., "Atomic physics techniques for studying nuclear ground state properties, fundamental interactions and symmetries: status and perspectives," Hyperfine Interactions 196, 295-337 (2010). 10.1007/s10751-010-0172-9.

[45] Puchalski, M. and Pachucki, K., "Nuclear structure effects in the isotope shift with halo nuclei," Hyperfine Interactions 196, 35-42 (2010). 10.1007/s10751-009-0137-z.

[46] van Rooij, R., Borbely, J. S., Simonet, J., Hoogerland, M. D., Eikema, K. S. E., Rozendaal, R. A., and Vassen, W., "Frequency metrology in quantum degenerate helium: Direct measurement of the $2{ }^{3} S_{1} \rightarrow 2{ }^{1} S_{0}$ transition," Science 333(6039), 196-198 (2011).

[47] Nörtershäuser, W., Neff, T., Sánchez, R., and Sick, I., "Charge radii and ground state structure of lithium isotopes: Experiment and theory reexamined," Phys. Rev. C 84, 024307 (Aug 2011). 
[48] Nörtershäuser, W., Tiedemann, D., Žáková, M., Andjelkovic, Z., Blaum, K., Bissell, M. L., Cazan, R., Drake, G. W. F., Geppert, C., Kowalska, M., Krämer, J., Krieger, A., Neugart, R., Sánchez, R., Schmidt-Kaler, F., Yan, Z.-C., Yordanov, D. T., and Zimmermann, C., "Nuclear charge radii of ${ }^{7,9,10} \mathrm{Be}$ and the one-neutron halo nucleus ${ }^{11}$ Be," Phys. Rev. Lett. 102, 062503 (Feb 2009).

[49] Sick, I. and Trautmann, D., "On the rms radius of the deuteron," Nuclear Physics A $637(4), 559-575$ (1998).

[50] Schmidt-Kaler, F., Leibfried, D., Weitz, M., and Hänsch, T. W., "Precision measurement of the isotope shift of the $1 S-2 S$ transition of atomic hydrogen and deuterium," Phys. Rev. Lett. 70, 2261-2264 (Apr 1993).

[51] Pachucki, K., Leibfried, D., Weitz, M., Huber, A., König, W., and Hänsch, T. W., "Theory of the energy levels and precise two-photon spectroscopy of atomic hydrogen and deuterium," Journal of Physics B: Atomic, Molecular and Optical Physics 29(2), 177 (1996).

[52] Pachucki, K., "Radiative recoil correction to the Lamb shift," Phys. Rev. A 52, 10791085 (Aug 1995).

[53] Puchalski, M. and Pachucki, K., "Relativistic, QED, and finite nuclear mass corrections for low-lying states of $\mathrm{Li}$ and $\mathrm{Be}^{+}, "$ Phys. Rev. A 78, 052511 (Nov 2008).

[54] Friar, J. L., Martorell, J., and Sprung, D. W. L., "Hadronic vacuum polarization and the Lamb shift," Phys. Rev. A 59, 4061-4063 (May 1999).

[55] Pachucki, K., "Nuclear structure corrections in muonic deuterium," Phys. Rev. Lett. 106, 193007 (May 2011).

[56] Udem, Th., Reichert, J., Hänsch, T. W., and Kourogi, M., "Accuracy of optical frequency comb generators and optical frequency interval divider chains," Opt. Lett. 23, 1387-1389 (Sep 1998).

[57] Benkler, E., Telle, H., Zach, A., and Tauser, F., "Circumvention of noise contributions in fiber laser based frequency combs," Opt. Express 13, 5662-5668 (Jul 2005).

[58] Kolachevsky, N., Haas, M., Jentschura, U. D., Herrmann, M., Fendel, P., Fischer, M., Holzwarth, R., Udem, Th., Keitel, C. H., and Hänsch, T. W., "Photoionization broadening of the $1 S-2 S$ transition in a beam of atomic hydrogen," Phys. Rev. A 74, 052504 (Nov 2006).

[59] McIntyre, D. H., Fairbank, W. M., Lee, S. A., Hänsch, T. W., and Riis, E., "Interferometric frequency measurement of ${ }^{130} \mathrm{Te}_{2}$ reference transitions at $486 \mathrm{~nm}$," Phys. Rev. A 41, 4632-4635 (May 1990). 
[60] Killian, T. C., Fried, D. G., Willmann, L., Landhuis, D., Moss, S. C., Greytak, T. J., and Kleppner, D., "Cold collision frequency shift of the $1 S-2 S$ transition in hydrogen," Phys. Rev. Lett. 81, 3807-3810 (Nov 1998).

[61] Parthey, C. G., Matveev, A., Alnis, J., Pohl, R., Udem, Th., Jentschura, U. D., Kolachevsky, N., and Hänsch, T. W., "Precision measurement of the hydrogen-deuterium $1 S-2 S$ isotope shift," Phys. Rev. Lett. 104, 233001 (Jun 2010).

[62] Barker, W. A. and Glover, F. N., "Reduction of relativistic two-particle wave equations to approximate forms. iii," Phys. Rev. 99, 317-324 (Jul 1955).

[63] Pachucki, K. and Karshenboim, S. G., "Nuclear-spin-dependent recoil correction to the Lamb shift," Journal of Physics B: Atomic, Molecular and Optical Physics 28(7), L221 (1995).

[64] Friar, J. L., Martorell, J., and Sprung, D. W. L., "Nuclear sizes and the isotope shift," Phys. Rev. A 56, 4579-4586 (Dec 1997).

[65] Czarnecki, A. and Melnikov, K., "Expansion of bound-state energies in powers of m/M," Phys. Rev. Lett. 87, 013001 (Jun 2001).

[66] Eides, M. I., Grotch, H., and Shelyuto, V. A., "Radiative-recoil corrections of order $\alpha(Z \alpha)^{5}(m / M) m$ to the Lamb shift revisited," Phys. Rev. A 63, 052509 (Apr 2001).

[67] Pachucki, K., "Radiative recoil correction to the Lamb shift," Phys. Rev. A 52, 10791085 (Aug 1995).

[68] Pachucki, K. private communication.

[69] Kopecky, S., Riehs, P., Harvey, J. A., and Hill, N. W., "New measurement of the charge radius of the neutron," Phys. Rev. Lett. 74, 2427-2430 (Mar 1995).

[70] Kopecky, S., Harvey, J. A., Hill, N. W., Krenn, M., Pernicka, M., Riehs, P., and Steiner, S., "Neutron charge radius determined from the energy dependence of the neutron transmission of liquid ${ }^{208} \mathrm{~Pb}$ and ${ }^{209} \mathrm{Bi}$," Phys. Rev. C 56, 2229-2237 (Oct 1997).

[71] Biraben, F., "Spectroscopy of atomic hydrogen," The European Physical Journal Special Topics 172, 109-119 (2009). 10.1140/epjst/e2009-01045-3.

[72] Altschul, B., "Testing electron boost invariance with $1 S-2 S$ hydrogen spectroscopy," Phys. Rev. D 81, 041701 (Feb 2010).

[73] Gabrielse, G., [Fundamental symmetries, edited by P. Bloch, P. Pavlopoulos and R. Klapisch], Plenum Press (1987).

[74] Hänsch, T. W., Lee, S. A., Wallenstein, R., and Wieman, C., "Doppler-free two-photon spectroscopy of hydrogen $1 S-2 S$," Phys. Rev. Lett. 34, 307-309 (Feb 1975). 
[75] Haas, M., Jentschura, U. D., Keitel, C. H., Kolachevsky, N., Herrmann, M., Fendel, P., Fischer, M., Udem, Th., Holzwarth, R., Hänsch, T. W., Scully, M. O., and Agarwal, G. S., "Two-photon excitation dynamics in bound two-body Coulomb systems including ac stark shift and ionization," Phys. Rev. A 73, 052501 (May 2006).

[76] McIntyre, D. H., Beausoleil, R. G., Foot, C. J., Hildum, E. A., Couillaud, B., and Hänsch, T. W., "Continuous-wave measurement of the hydrogen $1 S-2 S$ transition frequency," Phys. Rev. A 39, 4591-4598 (May 1989).

[77] Farley, J. W. and Wing, W. H., "Accurate calculation of dynamic Stark shifts and depopulation rates of Rydberg energy levels induced by blackbody radiation. hydrogen, helium, and alkali-metal atoms," Phys. Rev. A 23, 2397-2424 (May 1981).

[78] Vanier, J. and Audoin, C., [The Quantum Physics of Atomic Frequency Standards, Volume 2], Institute of Physics Publishing (1982).

[79] Huber, W. A., "Höchstauflösende Präzisionsspektroskopie am atomaren Wasserstoff," MPQ report 227 (1997).

[80] Gibble, K., "Difference between a photon's momentum and an atom's recoil," Phys. Rev. Lett. 97, 073002 (Aug 2006).

[81] Kostelecký, V. A. and Potting, R., "CPT and strings," Nuclear Physics B 359(2-3), $545-570$ (1991).

[82] Kostelecký, V. A. and Samuel, S., "Spontaneous breaking of Lorentz symmetry in string theory," Phys. Rev. D 39, 683-685 (Jan 1989).

[83] Gambini, R. and Pullin, J., "Nonstandard optics from quantum space-time," Phys. Rev. D 59, 124021 (May 1999).

[84] Alfaro, J., Morales-Técotl, H. A., and Urrutia, L. F., "Loop quantum gravity and light propagation," Phys. Rev. D 65, 103509 (Apr 2002).

[85] Kostelecký, V. A. and Russell, N., "Data tables for Lorentz and CPT violation," Rev. Mod. Phys. 83, 11-32 (Mar 2011).

[86] Kostelecký, V. A. and Lehnert, R., "Stability, causality, and Lorentz and CPT violation," Phys. Rev. D 63, 065008 (Feb 2001).

[87] Wolf, P., Chapelet, F., Bize, S., and Clairon, A., "Cold atom clock test of Lorentz invariance in the matter sector," Phys. Rev. Lett. 96, 060801 (Feb 2006).

[88] Matveev, A., Parthey, C. G., Katharina, P., Alnis, J., Beyer, A., Holzwarth, R., Udem, Th., Wilken, T., Kolachevsky, N., Abgrall, M., Rovera, D., Salomon, C., Laurent, P., Grosche, G., Legero, T., Schnatz, H., Weyers, S., Altschul, B., and Hänsch, T. W., "Limiting electron Lorentz boost invariance violation with hydrogen $1 S-2 S$," in preparation. 
[89] Vanhaecke, N., Meier, U., Andrist, M., Meier, B. H., and Merkt, F., "Multistage Zeeman deceleration of hydrogen atoms," Phys. Rev. A 75, 031402 (Mar 2007).

[90] Hogan, S. D., Wiederkehr, A. W., Schmutz, H., and Merkt, F., "Magnetic trapping of hydrogen after multistage Zeeman deceleration," Phys. Rev. Lett. 101, 143001 (Sep 2008).

[91] Narevicius, E., Libson, A., Parthey, C. G., Chavez, I., Narevicius, J., Even, U., and Raizen, M. G., "Stopping supersonic beams with a series of pulsed electromagnetic coils: An atomic coilgun," Phys. Rev. Lett. 100, 093003 (Mar 2008).

[92] Narevicius, E., Libson, A., Parthey, C. G., Chavez, I., Narevicius, J., Even, U., and Raizen, M. G., "Stopping supersonic oxygen with a series of pulsed electromagnetic coils: A molecular coilgun," Phys. Rev. A 77, 051401 (May 2008).

[93] Coherent Inc., "Coherent 699 manual,". 



\section{Acknowledgments}

As everybody working in experimental physics and probably most other natural sciences as well can confirm, research is not a one man show but rather team work. The work presented in this thesis could not have been completed without the help and advice of my colleagues and friends both at MPQ and around the world.

Dear Professor Hänsch, thank you for giving me the opportunity of working in your group. You have provided me with great freedom and the associated trust for developing my own ideas and have always been there offering your advice and encouragement. It has been a pleasure working in your group which provides a great atmosphere through very talented people using great equipment. Also, I want to take the opportunity to thank you for establishing the annual group seminar at the Ringberg Castle near Tegernsee as an intellectual retreet. A lot of creative solutions were created at this place, for example the breakthrough idea for measuring the $2 \mathrm{~S}$ velocity distribution was developed there.

Thomas Udem is the head of our MPQ group. Thomas, I profited a lot from you unique way of explaining things very clearly, sometimes even without being asked. We had a lot of fruitful discussions and you asked the right questions. Besides work, we share the passion for alpinism and you introduced me to extensive glacier hikes when taking me to Großer Löffler. Thank you for supporting me in any possible way, I very much enjoyed your company both at work and my spare time.

Nikolai Kolachevsky is visiting MPQ twice a year from Moscow. Nikolai, although you are only spending a third of your time at MPQ, your experience and long term memory of the experiment have been of immeasurable value to me. You introduced me to the peculiarities of the experiment and gave me a better understanding of what to expect from it. Later, you brought in innumerable fresh ideas and we developed a lot of strategies. Also, I enjoyed our late night discussions about the meaning of life, the universe and everything.

Arthur Matveev graduated during my time at MPQ. Arthur, we teamed up to manage the experiment and found a good working strategy to make it through two intensive measurement campaigns without completely burning our resources. Your good theoretical understanding was very helpful when searching for experimental strategies. I had a good time working with you. 
Janis Alnis is the senior postdoc with the hydrogen experiment. Janis, whenever something "fell out of lock" you are the person who can fix it most quickly. You have build the laser system enabling the experiments reported on in this theses. I envy your patience and want to thank you for your supportive attitude.

Randolf Pohl is among the most experience researchers in our group. Randolf, whenever I got stuck with a data evaluation problem you were the expert to talk to. Also, you are the group's window to the particle accelerator community and it was great fun visiting your experiment at Paul-Scherrer-Institute and two conferences. Thank you for all the time and endless coffee you provided me with during our discussions.

Axel Beyer joined our team as a master student about a year ago. Axel, it was great fun working with you and seeing you understand the lab more and more every day. From the first day on you stayed in the lab until late at night and it was because of you that the November measurement was much easier on Arthur and me than the one in May. Going out for a beer with you usually sucked since you have mostly taken away the girls' attention from me. I want to thank you for your engagement right from the start and wish you good luck in continuing the work we have started together.

Maximilian Herrmann is my climbing partner, an excellent physicist and a former postdoc at MPQ. Max, your broad interest and expertise in physics helped me to significantly speed up many projects. Naturally, I enjoyed our legendary road trips to the best climbing spots throughout the alps. I also want to thank your for introducing me to ice climbing which really helps me to bear the cold parts of the winter during which rock climbing is not an option.

Katharina Predehl established the fiber link between MPQ and PTB. Katha, we went through hours of discussing cycle slip schemes and while for me it was easy to implement them in the hydrogen lab, you had to communicate our new insights to PTB as well. Thanks for going through all the trouble and making the November measurement such a great success. I enjoyed our various climbing trips (except one...) and I should not forget to thank you and Birgitta for reserving the by far coolest film character in our 2008 Christmas movie for me - and providing me with one of the main characters in 2011.

Tobias Nebel graduated at the muonic hydrogen project during my time at MPQ. Tobi, thanks for showing me how to combine research and family, being at work early and how to use unusable Linux programs. Also, I don't want to miss our lunch conversations about life, not the universe and Apple gadgets. For a crazy two months we even managed to go bouldering early before work. However, in the end we realized that it was a stupid idea...

Tobias Wilken did his PhD in the astro-comb project at MPQ. Tobias, you re-locked and re-modelocked the frequency comb in the hydrogen lab close to a million times for us. 
I also want to thank you for explaining all the technical subtleties of this system in a very clear way and for going climbing with me every once in a while.

Johannes Hoffrogge spent hour after hour sitting back-to-back with me in the office. Johannes, besides our long debates about how to implement algorithms most efficiently in Matlab I enjoyed our discussions about mountain equipment safety and our trips to Franken Jura for some serious rock climbing.

Ronald Holzwarth is the head of Menlo Systems and an adviser to hour group. Ronald, while founding and running Menlo Systems you kept your interest and engagement with Physics. Your frequent advise with the frequency measurement was invaluable to me.

Marc Fischer is responsible for the frequency comb devision at Menlo Systems. Marc, when we needed a second frequency comb for the absolute frequency measurement you and your teams showed up at MPQ and installed a test comb within a few hours. Thank you!

There probably is not a single person within the Hänsch group that I did not profit from. I am grateful to all of you for contributing to the great group atmosphere.

Philippe Laurent, Daniele Rovera, and Michel Abgrall visited MPQ with their mobile fountain clock. Guys, I want to thank you for squeezing the hydrogen experiment in between two different campaigns on such short notice. I enjoyed having you at MPQ and while it was a quite exhausting time, we found time to explain our experiments to each other and somehow, we even managed to do slacklining and go to the mountains.

With Ulrich Jentschura we collaborated to extract the deuteron - proton charge radius difference from the isotope shift measurement. Ulrich, I want to thank you for introducing me to the tight jungle of QED contributions to the hydrogen spectrum. I cannot thank you enough for breaking down the contributions in a very intuitive and instructive way which I took over to this Thesis.

Brett Altschul collaborates with us on the electron Lorentz boost invariance test. Brett, we had a good time during your visit to MPQ and I want to thank you for your patience in explaining the general concepts of the standard model extensions to us total new bees in the field.

Harald Schnatz is the PTB end of the optical fiber link and Stefan Weyers operates the cesium fountain clocks at PTB. Harald and Stefan, I want to thank you for your hard work in providing the frequency reference during the November measurement.

I met Ed Narevicius in Austin as postdoc in Mark Raizen's group. While our ways split we always kept in touch. Ed, I want to thank you for inviting me to Weizmann Institute and providing me with a software package to understand the problem of decelerating hy- 
drogen with an atomic coilgun. You have always given me good advice and I enjoy your company. Thank you and Julia for letting me be your guest while in Israel. Keep visiting conferences in Munich!

I met quite a lot of people during conferences and our annual Ringberg meeting. I profited a lot from discussions and spontaneous lectures with and from these people. I would like to thank Eric Hessels, Krzysztof Pachucki, Christophe Salomon, Peter Mohr and Jim Bergquist.

I want to thank our group technicians Wolfgang Simon and Charlie Linner for their invaluable support and the ability to laugh about comments like "Can you finish this part until yesterday?". The same is true for our electrical engineer Helmuth Brückner whom I thank for overcompensating my lack of knowledge in electronics.

No group could work without administrative support. I want to thank Ingrid Hermann for managing numerous travels, visits and payment issues for me. I am grateful to Gabriele Gschwendtner helping me out in no time whenever I needed something at university.

I am deeply grateful to Andreas Widhopf who is the head of MPQ's building service. He and his team managed to allow the coexistence of precision measurements and noise and vibration intensive construction work by coordinating our measurement plans with the tight deadlines in renovating the labs right above the hydrogen lab.

I want to thank the teams of the purchasing and shipping departments who guaranteed a constant supply with new equipment. I am particularly grateful for the special service of having private packages being delivered to MPQ.

I also want to thank my family. My parents, Almuth and Roland, have always supported my passion for physics. So did my brother Matthias and my sister Johanna as well as my aunt Siegrid, uncle Godehard, and my granny Erika. Thank you for always being there for me.

Uli, thank you for helping me to find my place in life. 


\section{List of Publications}

1. E. Narevicius, A. Libson, M.F. Riedel, C.G. Parthey, I. Chavez, U. Even, and M.G. Raizen, Coherent Slowing of a Supersonic Beam with an Atomic Paddle, Phys. Rev. Lett. 98, 103201 (2007)

2. E. Narevicius, C.G. Parthey, A. Libson, M.F. Riedel, U. Even and M.G. Raizen, Towards magnetic slowing of atoms and molecules, New J. Phys. 9, 96 (2007)

3. E. Narevicius, C.G. Parthey, A. Libson, J. Narevicius, I. Chavez, U. Even and M.G. Raizen, An atomic coilgun: using pulsed magnetic fields to slow a supersonic beam, New J. Phys. 9, 358 (2007)

4. E. Narevicius, A. Libson, C.G. Parthey, I. Chavez, J. Narevicius, U. Even and M.G. Raizen, Stopping Supersonic Beams with a Series of Pulsed Electromagnetic Coils: An Atomic Coilgun, Phys. Rev. Lett. 100, 093003 (2008)

5. E. Narevicius, A. Libson, C.G. Parthey, I. Chavez, J. Narevicius, U. Even and M.G. Raizen, Stopping supersonic oxygen with a series of pulsed electromagnetic coils: A molecular coilgun, Phys. Rev. A 77, 051401(R) (2008)

6. N. Kolachevsky, A. Matveev, J. Alnis, C.G. Parthey, S.G. Karshenboim, and T.W. Hänsch, Measurement of the $2 S$ Hyperfine Interval in Atomic Hydrogen, Phys. Rev. Lett. 102, 213002 (2009)

7. N. Kolachevsky, A. Matveev, J. Alnis, C.G. Parthey, T. Steinmetz, T. Wilken, R. Holtzwarth, Th. Udem, and T.W. Hänsch, Testing the Stability of the Fine Structure Constant in the Laboratory, Space Sci. Rev. 148, 267 (2009)

8. C.G. Parthey, A. Matveev, J. Alnis, R. Pohl, Th. Udem, U.D. Jentschura, N. Kolachevsky, and T.W. Hänsch, Precision Measurement of the Hydrogen-Deuterium 1S-2S Isotope Shift, Phys. Rev. Lett. 104, 233001 (2010)

9. U.D. Jentschura, A. Matveev, C.G. Parthey, J. Alnis, R. Pohl, Th. Udem, N. Kolachevsky, and T.W. Hänsch, Hydrogen-deuterium isotope shift: From the $1 S-2 S$ transition frequency to the proton-deuteron charge-radius difference, Phys. Rev. A. 83, 042505 (2011) 
10. N. Kolachevsky, J. Alnis, C.G. Parthey, A. Matveev, R. Landig, T.W. Hänsch, Low phase noise diode laser oscillator for $1 S-2 S$ spectroscopy in atomic hydrogen, Opt. Lett. 36, 4299 (2011)

11. C.G. Parthey, A. Matveev, J. Alnis, B. Bernhardt, A. Beyer, R. Holzwarth, A. Maistrou, R. Pohl, K. Predehl, Th. Udem, T. Wilken, N. Kolachevsky, M. Abgrall, D. Rovera, C. Salomon, P. Laurent, and T.W. Hänsch, Improved Measurement of the Hydrogen 1S-2S Transition Frequency, Phys. Rev. Lett. 107, 203001 (2011)

12. C.G. Parthey, A. Matveev, J. Alnis, A. Beyer, R. Pohl, K. Predehl, Th. Udem, N. Kolachevsky, M. Abgrall, D. Rovera, C. Salomon, P. Laurent, and T. W. Hänsch, Precision spectroscopy on atomic hydrogen, Proc. SPIE 8132, 813202 (2011)

\section{Invited Talks}

2011 Colloquium of the Max-Planck-Institut für Quantenoptik, Garching 2011 Astronomy, Clocks and Fundamental Constants, Bad Honnef 2011 SPIE Optics + Photonics, San Diego, CA

\section{Conference Contributions}

2007 Meeting of the American Physical Society Texas Devision, College Station, TX

2009 10th French-Israeli Symposium on Non-linear and Quantum Optics, Ein Gedi, Israel

2010 International Conference on Atomic Physics (ICAP), Cairns, Australien

2011 Frühjahrstagung der Deutschen Physikalischen Gesellschaft, Dresden

2011 International Conference on Laser Spectroscopy (ICOLS), Aerzen 